\title{
Li abundances in F stars: planets, rotation, and Galactic evolution $\star, \star \star$
}

\author{
E. Delgado Mena ${ }^{1,2}$, S. Bertrán de Lis $s^{3,4}$, V. Zh. Adibekyan ${ }^{1,2}$, S. G. Sousa ${ }^{1,2}$, P. Figueira ${ }^{1,2}$, A. Mortier ${ }^{6}$, \\ J. I. González Hernández ${ }^{3,4}$, M. Tsantaki ${ }^{1,2,3}$, G. Israelian ${ }^{3,4}$, and N. C. Santos ${ }^{1,2,5}$ \\ ${ }^{1}$ Centro de Astrofisica, Universidade do Porto, Rua das Estrelas, 4150-762 Porto, Portugal \\ e-mail: Elisa.Delgado@astro.up.pt \\ 2 Instituto de Astrofísica e Ciências do Espaço, Universidade do Porto, CAUP, Rua das Estrelas, 4150-762 Porto, Portugal \\ 3 Instituto de Astrofísica de Canarias, C/via Lactea, s/n, 38200 La Laguna, Tenerife, Spain \\ ${ }^{4}$ Departamento de Astrofísica, Universidad de La Laguna, 38205 La Laguna, Tenerife, Spain \\ 5 Departamento de Física e Astronomía, Faculdade de Ciências, Universidade do Porto, Portugal \\ ${ }^{6}$ SUPA, School of Physics and Astronomy, University of St. Andrews, St. Andrews KY16 9SS, UK
}

Received 28 November 2014 / Accepted 14 December 2014

\section{ABSTRACT}

\begin{abstract}
Aims. We aim, on the one hand, to study the possible differences of Li abundances between planet hosts and stars without detected planets at effective temperatures hotter than the Sun, and on the other hand, to explore the Li dip and the evolution of Li at high metallicities.

Methods. We present lithium abundances for 353 main sequence stars with and without planets in the $T_{\text {eff }}$ range $5900-7200 \mathrm{~K}$. We observed 265 stars of our sample with HARPS spectrograph during different planets search programs. We observed the remaining targets with a variety of high-resolution spectrographs. The abundances are derived by a standard local thermodynamic equilibrium analysis using spectral synthesis with the code MOOG and a grid of Kurucz ATLAS9 atmospheres.

Results. We find that hot jupiter host stars within the $T_{\text {eff }}$ range $5900-6300 \mathrm{~K}$ show lower Li abundances, by 0.14 dex, than stars without detected planets. This offset has a significance at the level $7 \sigma$, pointing to a stronger effect of planet formation on $\mathrm{Li}$ abundances when the planets are more massive and migrate close to the star. However, we also find that the average $v \sin i$ of (a fraction of) stars with hot jupiters is higher on average than for single stars in the same $T_{\text {eff }}$ region, suggesting that rotational-induced mixing (and not the presence of planets) might be the cause for a greater depletion of Li. We confirm that the mass-metallicity dependence of the Li dip is extended towards $[\mathrm{Fe} / \mathrm{H}] \sim 0.4$ dex (beginning at $[\mathrm{Fe} / \mathrm{H}] \sim-0.4$ dex for our stars) and that probably reflects the mass-metallicity correlation of stars of the same $T_{\text {eff }}$ on the main sequence. We find that for the youngest stars $(<1.5 \mathrm{Gyr})$ around the Li dip, the depletion of $\mathrm{Li}$ increases with $v \sin i$ values, as proposed by rotationally-induced depletion models. This suggests that the Li dip consists of fast rotators at young ages whereas the most Li-depleted old stars show lower rotation rates (probably caused by the spin-down during their long lifes). We have also explored the Li evolution with $[\mathrm{Fe} / \mathrm{H}]$ taking advantage of the metal-rich stars included in our sample. We find that $\mathrm{Li}$ abundance reaches its maximum around solar metallicity, but decreases in the most metal-rich stars, as predicted by some models of Li Galactic production.
\end{abstract}

Key words. stars: abundances - stars: fundamental parameters - stars: rotation - stars: evolution - planets and satellites: formation planetary systems

\section{Introduction}

Lithium is one of the most studied chemical elements in the literature. Despite efforts to unveil the mechanisms of production and destruction of this interesting element, there are still some unsolved mysteries. For instance, the disagreement found between

* Based on observations collected at the La Silla Observatory, ESO (Chile), with the HARPS spectrograph at the $3.6 \mathrm{~m}$ ESO telescope, with CORALIE spectrograph at the $1.2 \mathrm{~m}$ Euler Swiss telescope and with the FEROS spectrograph at the $1.52 \mathrm{~m}$ ESO telescope; at the Paranal Observatory, ESO (Chile), using the UVES spectrograph at the VLT/UT2 Kueyen telescope, and with the FIES and SARG spectrographs at the $2.5 \mathrm{~m}$ NOT and the $3.6 \mathrm{~m} \mathrm{TNG}$, respectively, both at La Palma (Canary Islands, Spain).

$\star \star$ Tables 3-6 are available in electronic form at http://www. aanda.org the abundance of the most metal-poor stars in the Galaxy (the so-called "Spite plateau" with $A(\mathrm{Li})^{1} \sim 2.2$, Spite \& Spite 1982) and the initial primordial abundance given by the WMAP observations $(A(\mathrm{Li}) \sim 2.7$, Steigman 2010, Cyburt et al. 2008) is not understood yet. Moreover, the current Galactic Li production models (e.g. Prantzos 2012) are not able to yield enough Li to explain the meteoritic abundance of 3.31 (Anders \& Grevesse 1989) or the maximum $\mathrm{Li}$ abundances found in young clusters (e.g. Sestito \& Randich 2005). On the other hand, the standard model of Li depletion, which only considers convection (e.g. Deliyannis et al. 1990; Pinsonneault 1997) cannot explain the observed Li abundances in solar-type stars or in mid-F stars that have undergone the Li dip. Furthermore, in previous years a new discussion about the effect of planets on the depletion of Li has

$1 \quad A(\mathrm{Li})=\log [N(\mathrm{Li}) / N(\mathrm{H})]+12$. 
Table 1. Parameters for each coefficient as resulting from multivariable linear regression analysis in the four tests (PH are planet hosts and CS are the comparison stars).

\begin{tabular}{lccccccccc}
\hline \hline Sample of planet hosts & Number & Int. & $\beta_{1}$ & $\beta_{2}$ & $\beta_{3}$ & $\beta_{4}$ & $\beta_{5}$ & Offset & Significance \\
\hline Jupiter size hosts & 87PH \& 176CS & -64.04 & 17.81 & 0.02 & -0.18 & -0.21 & & -0.07 & $6.5 \sigma$ \\
Hot jupiter hosts & 24PH\& 176CS & -69.64 & 19.37 & -0.05 & -0.24 & -0.28 & & -0.14 & $7.0 \sigma$ \\
\hline Analysis including $v \sin i$ & & & & & & & & & \\
\hline Jupiter size hosts & 47PH \& 62CS & -86.39 & 23.72 & 0.06 & -0.10 & -0.20 & -0.12 & 0.04 & $2.6 \sigma$ \\
Hot jupiter hosts & 11PH \& 62CS & -98.37 & 27.16 & -0.05 & -0.29 & -0.28 & -0.15 & 0.08 & $2.1 \sigma$ \\
\hline
\end{tabular}

Notes. The offset is only included in the fit for planet hosts since the offset for comparison stars is by definition 0 . The last column reflects the siginificance of the offset found between both samples.

been opened (e.g. Israelian et al. 2009; Ramírez et al. 2012; Gonzalez 2014; Figueira et al. 2014; Delgado Mena et al. 2014, hereafter DM14).

Lithium, as other light elements, can easily be destroyed in stellar interiors by $\mathrm{p}-\alpha$ reactions. Although Li depletion occurs primarily in the pre-main sequence (PMS), it can also take place in stellar envelopes if there is any extra mixing process. Therefore, Li abundance can provide us important information about the internal structure of stars. In this work we present homogeneous Li abundances for a sample of 353 "hot" stars (early $\mathrm{G}$ and $\mathrm{F}$ stars) with a wide range of metallicities and ages. We exploit the metal-rich stars in our sample to study the behaviour of the Li dip and the chemical evolution of Li at high metallicities. Finally, we also investigate if the presence of planets affect $\mathrm{Li}$ abundances for these hotter stars.

Section 2 briefly describes the collected data together with the determination of stellar parameters and abundances of lithium. In Sect. 3 we discuss the results related to different topics: the connection of $\mathrm{Li}$ abundances with the presence of planets, the behaviour of the $\mathrm{Li} \mathrm{dip}$, the chemical evolution of $\mathrm{Li}$ at high metallicities, and the Li distribution in the Galactic disks. We summarize in Sect. 4.

\section{Observations and analysis}

Our baseline sample is 1111 FGK stars observed within the context of the HARPS GTO programs. It is a combination of three HARPS subsamples hereafter called HARPS-1 (Mayor et al. 2003), HARPS-2 (Lo Curto et al. 2010), and HARPS-4 (Santos et al. 2011). The individual spectra of each star were reduced using the HARPS pipeline and then combined with IRAF $^{2}$ after correcting for its radial velocity shift. The final spectra have a resolution of $R \sim 110000$ and high signal-to-noise ratio $(55 \%$ of the spectra have a $\mathrm{S} / \mathrm{N}$ higher than 200). The total sample is composed of 135 stars with planets and 976 stars without detected planets. For this work, we mainly focus on the hottest $T_{\text {eff }}$ $(>5900 \mathrm{~K})$ where we have 36 and 229 stars with and without planets, respectively. All the planet hosts and non-hosts stars are listed in Tables 3 and 4, respectively. To increase the number of stars with planets, we used high-resolution spectra for 88 planet hosts (see Table 5) that come from different observing runs and spectrographs. Table 1 of DM14 lists those instruments in detail. The data reduction was made using the IRAF package or the respective telescopes pipelines. All the images were flat-field corrected, sky substracted, and co-added to obtain 1D spectra. Doppler correction was also done.

\footnotetext{
2 IRAF is distributed by National Optical Astronomy Observatories, operated by the Association of Universities for Research in Astronomy, Inc., under contract with the National Science Foundation, USA.
}

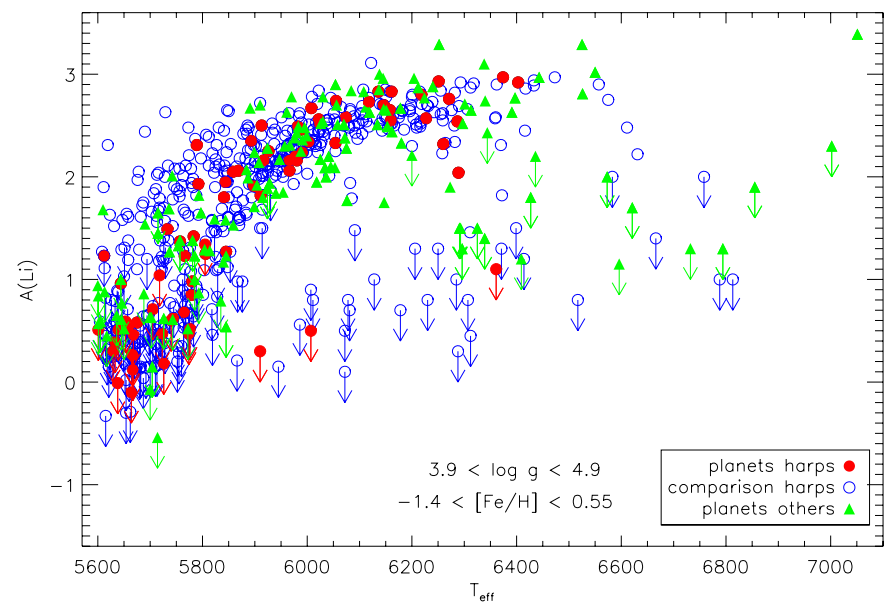

Fig. 1. Lithium abundances vs. $T_{\text {eff }}$ for planet host stars (red filled circles) and comparison sample stars (blue open circles) from HARPS together with other planet hosts (green triangles). Down arrows represent $A$ (Li) upper limits.

The stellar atmospheric parameters were taken from Sousa et al. (2008, 2011a,b) for the HARPS samples and from Santos et al. (2004, 2005), Sousa et al. (2006), Mortier et al. (2013), Santos et al. (2013) for the rest of the planet hosts. All the sets of parameters were determined in a homogeneous way. We derived lithium abundances $A(\mathrm{Li})$, stellar masses and ages in the same way as DM14. That work offers further details about the determination of stellar parameters and $\mathrm{Li}$ abundances.

The determination of rotational projected velocities ( $v \sin i$ values) was done with a combined Fourier transform and goodness-of-fit methodology using the IACOB program (Simón-Díaz \& Herrero 2014). We could only determine $v \sin i$ for the stars with spectra of S/Ns above $\sim 100$.

\section{Results and discussion}

\subsection{General behaviour of Li in F stars}

In Fig. 1 we present a general overview of the Li abundances as a function of effective temperature for our sample. The ranges in $[\mathrm{Fe} / \mathrm{H}]$ and gravity for the stars in this sample are specified in the plot. To better appreciate the behaviour of $\mathrm{Li}$ in a wider $T_{\text {eff }}$ range, we have also included the solar-type stars from DM14, with $5600 \mathrm{~K}<T_{\text {eff }}<5900 \mathrm{~K}$. As expected, Li abundances decrease as the stars get cooler because of their thicker convective envelopes. However, for higher $T_{\text {eff }}$, we still observe an important number of stars with a strong destruction of Li. The stars around $6400 \mathrm{~K}$ belong to the well-known $\mathrm{Li}$ dip, which was first discovered in the Hyades cluster by Boesgaard \& Tripicco (1986), but those with cooler temperatures, between $5900 \mathrm{~K}$ 


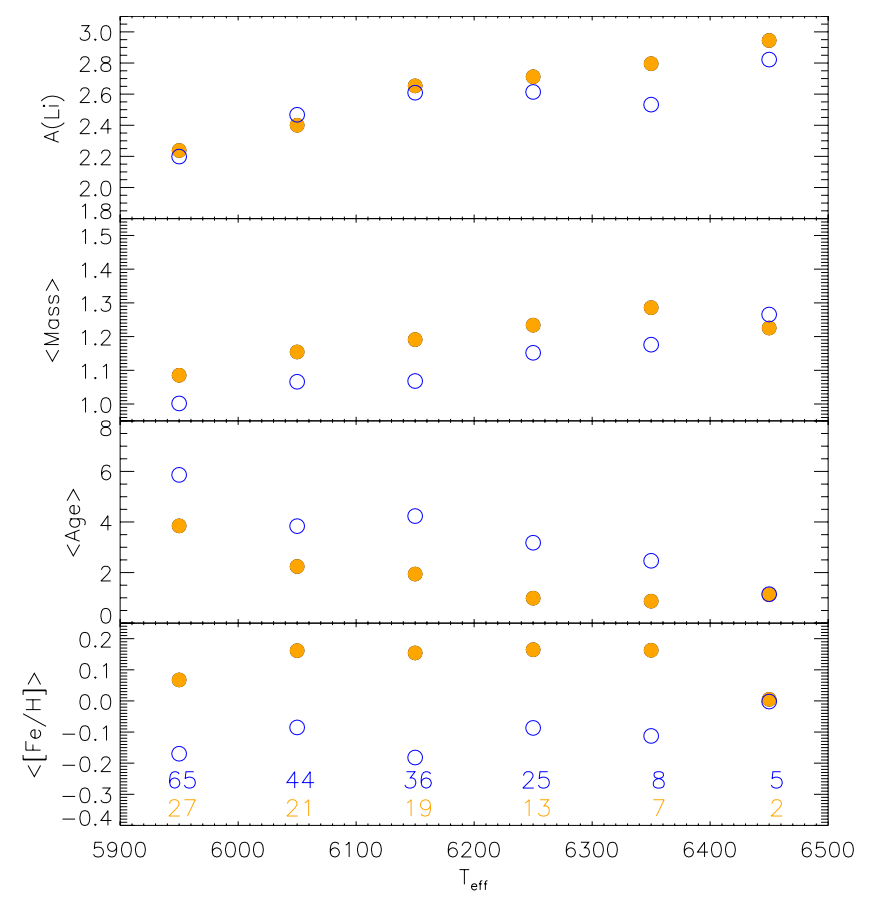

Fig. 2. Average of $A(\mathrm{Li})$, stellar mass, age, and $[\mathrm{Fe} / \mathrm{H}]$ in six $T_{\text {eff }}$ bins for all (HARPS+others) the planet host stars (orange filled circles) and comparison sample stars (blue open circles). The number of stars in each bin is indicated with the respective colour. Only stars with Li detections are considered.

and $6200 \mathrm{~K}$, are not so common in studies of clusters or field stars. We would expect these stars to have higher Li abundances unless they are evolved stars from the dip, as suggested by Chen et al. (2001). These objects will be further studied in a separate work. Although we do not expect to have evolved stars in our sample we have removed the stars with $\log g<4.2$ since our spectroscopic $\log g$ values could be overestimated for the hottest stars (Mortier et al. 2014).

\subsection{Li and planets}

The Li dependence on the presence of planets has been extensively discussed in the literature. On the one hand, several independent groups find that planet hosts with $T_{\text {eff }}$ close to solar present lower abundances of Li when compared to non-hosts (Israelian et al. 2004, 2009; Takeda \& Kawanomoto 2005; Chen \& Zhao 2006; Gonzalez 2008, 2014; Takeda et al. 2010; Gonzalez et al. 2010; Sousa et al. 2010; Delgado Mena et al. 2014; Figueira et al. 2014). On the other hand, other authors do not find such a dependence (Ryan 2000; Luck \& Heiter 2006; Baumann et al. 2010; Ghezzi et al. 2010; Ramírez et al. 2012). Gonzalez (2008) proposed that stars with planets around $6100 \mathrm{~K}$ show higher Li abundances than stars without detected planets. After increasing the sample size, however, the same author discarded this effect and presented weak evidence that planet hosts at $T_{\text {eff }} \sim 6100-6200 \mathrm{~K}$ are deficient in Li compared to stars without detected planets (Gonzalez 2014, 2015). Visually we cannot pinpoint any strong difference in the $\mathrm{Li}$ abundance detections between stars with and without planets in Fig. 1. However it is very clear that in the $T_{\text {eff }}$ range between $5900 \mathrm{~K}$ and $6300 \mathrm{~K}$ there are relatively more non-hosts with upper limits in $\mathrm{Li}$ abundances. This feature was also pointed out by Ramírez et al. (2012).

In Fig. 2 we compile the average values of $\mathrm{Li}$ abundance detections and other parameters for stars with and without detected planets (in bins of $100 \mathrm{~K}$ ). Since Li abundances depend on several parameters (e.g. $T_{\text {eff }},[\mathrm{Fe} / \mathrm{H}]$, age) one should be cautious when comparing stars and construct the least biased possible samples (for a further discussion see DM14). For example, in all these subsamples except the hottest one, planet hosts are younger and also on average more metal rich, as expected (e.g. Santos et al. 2004). Nevertheless, this difference in parameters does not seem to affect the degree of Li depletion too much (see Sect. 3.4), except maybe in the $T_{\text {eff }}$ range $6300-6400 \mathrm{~K}$ where we observe the highest difference in Li. For the rest of the subamples, the average values of stars with and without planets are quite similar and within the errors.

To remove the effect of different stellar parameters when comparing $\mathrm{Li}$ abundances, we apply a multivariate regression fit to the planet host sample and the comparison sample as done in Figueira et al. (2014):

$$
\begin{aligned}
\log (A(\mathrm{Li}))= & \text { int. }+\beta_{1} \log \left(T_{\text {eff }}\right)+\beta_{2}[\mathrm{Fe} / \mathrm{H}]+\beta_{3} \log g \\
& +\beta_{4} \log (\text { Age })+M \times \text { offset. }
\end{aligned}
$$

In both samples, we assumed the same linear dependence of $\mathrm{Li}$ on stellar parameters but allow an offset for the planet host sample, which is, $M=0$ for the comparison sample and $M=1$ for the planet host sample. By doing so, we ensure that a possible difference in Li abundance is not due to different stellar parameters. For this calculation we consider all our stars with $5900 \mathrm{~K}<$ $T_{\text {eff }}<6300 \mathrm{~K}$ and $\mathrm{Li}$ detections (we exclude the upper limits), 87 planet hosts (with Jupiter type planets: $M_{\mathrm{P}} \geq 0.1 M_{J}$ ) and 176 comparison stars. We chose to cut at $T_{\text {eff }}=6300 \mathrm{~K}$ to allow for a fair comparison with the Gonzalez (2015) sample. Moreover, that is roughly the temperature at which the Li dip begins to develop and it would be difficult to distinguish a possible effect of planets on $\mathrm{Li}$ abundances from other depletion mechanisms.

The results are shown in Table 1. As expected, the strongest dependence lies on $T_{\text {eff }}$, while it is very small for the other parameters. We find that the planet host sample shows a depletion of 0.07 dex with respect to non-hosts. Although this offset is significant (at $6.5 \sigma$ level), its significance is naturally heavily dependent on the error bars. If we artificially increase the error bars by a factor of 2 or 3 the significance drops to $3.3 \sigma$ and $2.2 \sigma$, respectively. This offset agrees with the results by Gonzalez (2015) though it is very small and at the level of the uncertainties $^{3}$. Therefore, it seems that the effect of giant planets observed for solar analogues is not obvious for hotter stars, probably because of their shallower convective envelopes. As explained in DM14, the effect of planets on Li abundances is expected to be higher for more massive planets (stronger effect on rotational history, Bouvier 2008), and for planets that migrate (more violent accretion bursts, Baraffe \& Chabrier 2010). Thus, we explore the behaviour of stars hosting hot jupiters since these planets are massive and some theories of planet formation predict a migration close to the star (e.g. Alibert et al. 2005). Then, we repeat our previous calculation, instead using as planet host sample only those stars that host planets with $M>0.1 M_{J}$ and $P<5$ days. In this case we find a higher offset than before, -0.14 dex, with a significance level of $7 \sigma$. As before, we increase the error bars by a factor of 2 and 3, which drops the significance to $3.6 \sigma$ and $2.4 \sigma$, respectively. We note that our sample of hot jupiter hosts is small ( 24 stars with $5900 \mathrm{~K}<T_{\text {eff }}<6300 \mathrm{~K}$ ), but this is an interesting result that deserves to be explored further in the future.

The average uncertainty in $\mathrm{Li}$ abundances for our stars is $0.07 \mathrm{dex}$. 


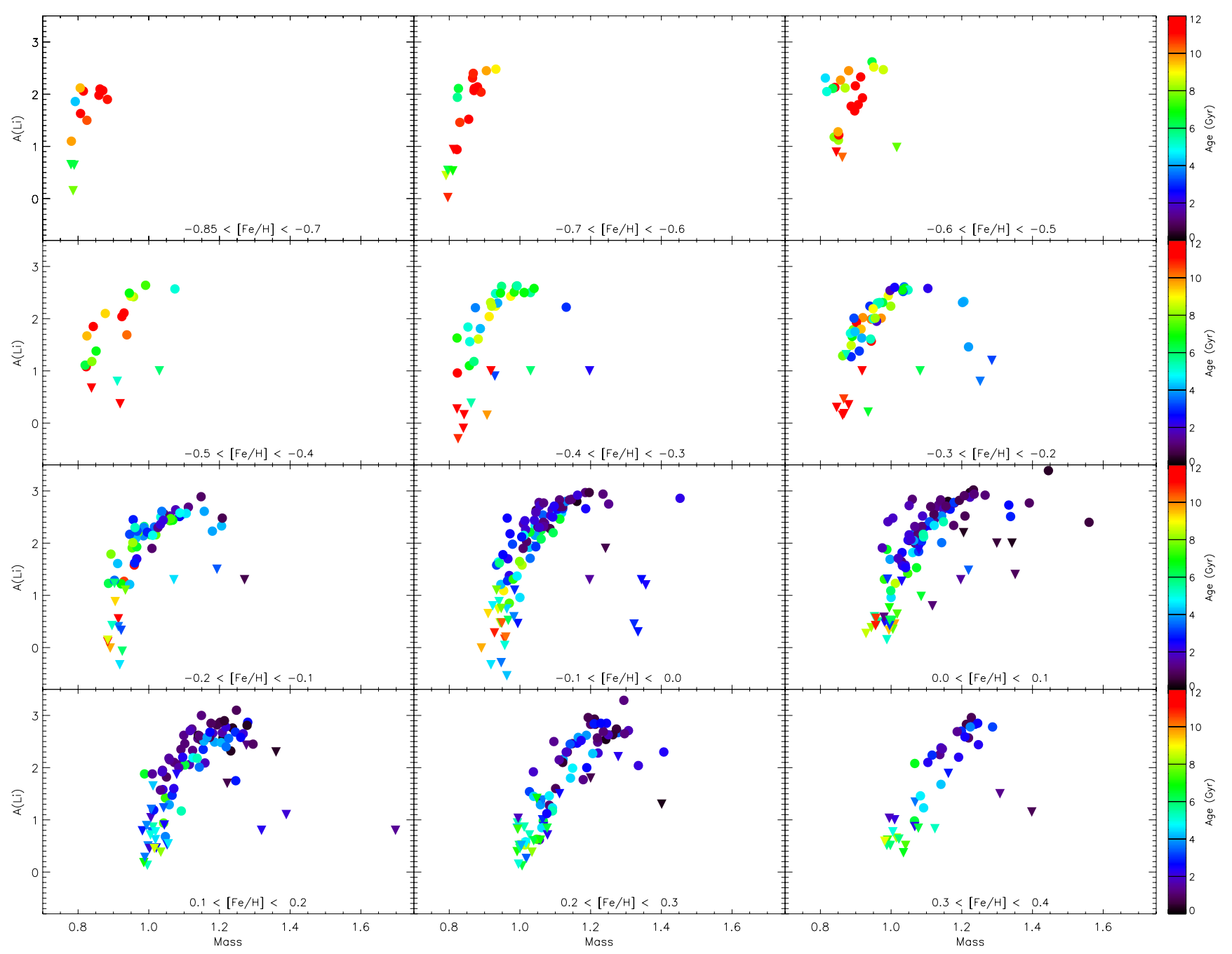

Fig. 3. Lithium abundances vs. stellar mass in 12 metallicity bins for all our stars with $\log g>4.2$. Downward triangles represent $A$ (Li) upper limits. The ages are depicted by a colour scale.

Finally, we investigate the possible effect of rotation on $\mathrm{Li}$ abundances for our sample of planet hosts. The models of rotationally-induced mixing predict that during the main sequence (MS), stars with higher rotation rates are expected to deplete more $\mathrm{Li}$ than slower rotators. We find that the average $v \sin i$ for hot jupiter hosts in this $T_{\text {eff }}$ range is larger, $5 \mathrm{~km} \mathrm{~s}^{-1}$ (derived only for 11 stars) than for the comparison stars, $3.1 \mathrm{~km} \mathrm{~s}^{-1}$ (derived for 62 stars), hence this could explain the offset previously found for stars with hot jupiters. In order to test this effect, we repeat the same analysis as before except we include the $v \sin i$ in the equation and force a same dependence on it both for the planet host sample and in the comparison stars sample

$\log (A(\mathrm{Li}))=$ int. $+\beta_{1} \log \left(T_{\text {eff }}\right)+\beta_{2}[\mathrm{Fe} / \mathrm{H}]+\beta_{3} \log g$

$$
+\beta_{4} \log (\mathrm{Age})+\beta_{5} v \sin i+M \times \text { offset. }
$$

The results are shown in the second part of Table 1 . As expected from the models of rotationally-induced mixing, Li abundances show a negative dependence on $v \sin i$. The offsets are now positive (i.e. higher Li abundances for planet hosts) but they are also much less significant than before $(2.6 \sigma$ and $2.1 \sigma$, for the jupiter size planets and the hot jupiters, respectively). This result points to an effect of rotation on $\mathrm{Li}$ abundances though we have to be cautious since our sample of measured $v \sin i$ values is very small and potentially biased (we could only measure $v \sin i$ in $42 \%$ of our stars with Li detections in this $T_{\text {eff }}$ range).

\subsection{The Li dip: dependence on metallicity, age and $v \sin i$}

The Li dip was first discovered in the Hyades by Boesgaard \& Tripicco (1986). For clusters younger than the Pleaides ( $200 \mathrm{Myr})$ this feature is not observed and stars more massive than a solar mass show a constant maximum value of $A(\mathrm{Li})=$ 3-3.2 dex (Lambert \& Reddy 2004). Therefore, the Li dip has to be formed during the MS. Indeed, the maximum $\mathrm{Li}$ abundance is similar for the youngest clusters as for the slightly older clusters (300 Myr-2 Gyr), hence, F stars in the MS experience very little depletion up to ages of 1 Gyr. For older clusters there are hints of the presence of the dip though there is not always a significant number of stars at those temperatures. The Li dip is not well defined in Fig. 1 because in our sample there are stars of different ages and metallicities. To appreciate a clearly shaped Li dip one should divide the stars by ranges with similar $[\mathrm{Fe} / \mathrm{H}]$ and age, as happens in open clusters.

In Fig. 3 we show $\mathrm{Li}$ abundances as a function of mass in 12 different metallicity bins for the stars in this work together with the sample of DM14 (we exclude the most metalpoor and most metal-rich bins due to their low number of stars). From this plot we can confirm that the Li dip happens at higher masses as the metallicity increases. This fact was first suggested by Balachandran (1995), who found that the mass at which the dip occurs depends on the stellar metallicity, while the zero age 
main sequence (ZAMS) $T_{\text {eff }}$ does not. Later studies on clusters (e.g. Cummings et al. 2012; François et al. 2013) or field stars (e.g. Lambert \& Reddy 2004) have confirmed this feature. For example in the $[\mathrm{Fe} / \mathrm{H}]$ range $[-0.6,-0.5]$, we have a unique star at the Li dip with a mass of $1.02 M_{\odot}$. This agrees with the Li dip centre of $1.06 M_{\odot}$ found by (François et al. 2013) in a similar metallicity cluster, NGC 2243 with $[\mathrm{Fe} / \mathrm{H}]=-0.54$ dex. By contrast, at higher metallicities we find two stars with 1.3 and $1.4 M_{\odot}$ in the Li dip, which compares well with the mass of the cool side of Li dip in NGC $6253\left(1.34 M_{\odot},[\mathrm{Fe} / \mathrm{H}]=0.43\right.$ dex, Cummings et al. 2012). In that work they also compare their results with the Hyades, which has a Li dip mass of $1.27 M_{\odot}$. In our field stars of similar metallicity (0.1-0.2 dex), the Li dip seems to be at $\sim 1.3 M_{\odot}$. From Fig. 3 we can see that the increase of mass with metallicity not only happens for the Li dip stars but for all the objects within each metallicity bin, therefore this is just a reflection of the mass-metallicity correlation for stars with similar $T_{\text {eff }}$ in the MS and confirms the suggestion by Balachandran (1995).

In Fig. 3 we also show the ages of the stars by a colour scale. For the more metal-poor bins we cannot observe the Li dip because our MS stars are too old and thus too cool to be susceptible to that process. For instance, for older clusters like M67 the Li dip is formed by subgiants. We have cleaned our sample of possible evolved stars so the hotter stars that usually form the Li dip have to be young enough and not evolved yet. In fact, if we observe the Li dip at different metallicities it is always formed by stars younger than $\sim 4 \mathrm{Gyr}$, with the age slightly decreasing as the metallicity increases.

Several mechanisms have been proposed to explain the formation of the Li dip, such as mass loss (Schramm et al. 1990), diffusion, and radiative acceleration (Richer \& Michaud 1993), or rotationally induced mixing (Zahn 1992; Pinsonneault et al. 1990). Under the assumption of this last mechanism, stars that rotate faster in the MS will experience more rapid mixing (thus, more Li depletion) than slow rotators at the same mass (Pinsonneault 1997). In principle, this seems to be at odds with the work by Bouvier (2008), where the slow rotators on the ZAMS suffer a stronger depletion of Li than fast rotators, and with the higher Li abundances found in rapidly rotating stars as compared with slow rotators of the same mass in the Pleiades (Soderblom et al. 1993; Garcia Lopez et al. 1994) or IC 2602 (Randich et al. 1997). However, at this point one has to be careful distinguishing between the depletion mechanisms acting during the MS and the PMS (e.g. Somers \& Pinsonneault 2014) and between the current rotation rates and those in the ZAMS, though a priori one could expect that a star with a current high velocity was also a fast rotator in the past. Nevertheless, it is difficult to estimate the initial rotation velocity since stars usually spin down when arriving at the MS. Therefore we have to extract information from the current surface rotation rates. Moreover, for most of the stars, we do not know their inclination so we can only measure the projected rotational velocity, $v \sin i$, given that we have a good quality spectrum.

To check for the possible impact of rotation, we compare the $v \sin i$ values of the stars that typically form the Li gap (which we define in the range $6280 \mathrm{~K}<T_{\text {eff }}<6550 \mathrm{~K}$, see Fig. 4). We note that in this plot the number of stars is lower since we were able to determine $v \sin i$ for only 20 out of 50 stars in this $T_{\text {eff }}$ range. To increase the statistics we included 12 fast rotators within the same $T_{\text {eff }}$ range analysed in Tsantaki et al. (2014), (see Table 2). For those stars the stellar parameters are derived using the spectral synthesis technique for FGK stars and are in agreement with the results of the EW method. In addition,

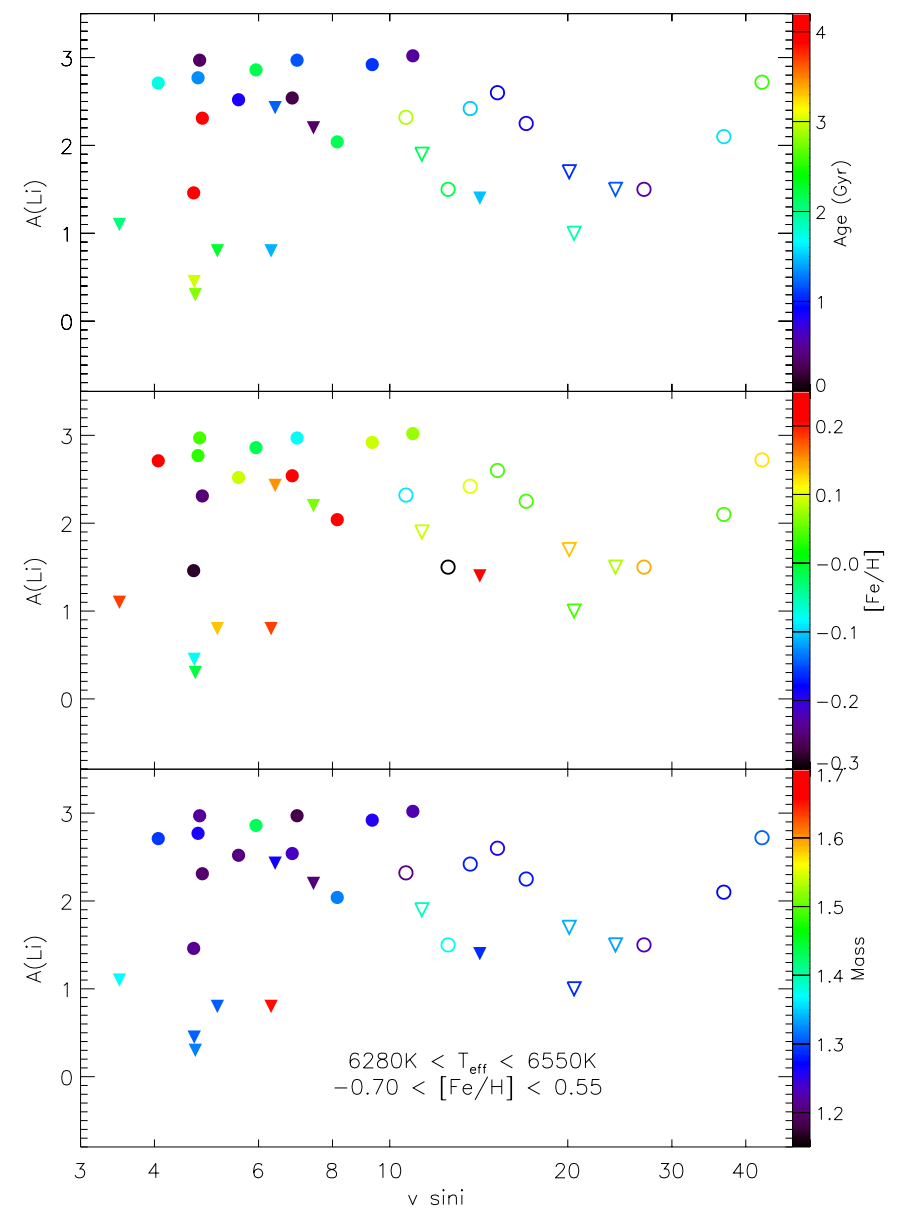

Fig. 4. Lithium abundances vs. $v \sin i$ around the Li dip for all our stars (filled circles and triangles). Open circles and triangles (upper limits) represent the fast rotators from Tsantaki et al. (2014). In each panel a colour scale shows the ages, metallicities, and masses of the stars.

the comparison of $v \sin i$ from the spectral synthesis technique mentioned above and our method shows a good agreement. The $\mathrm{Li}$ abundances for those 12 objects were derived in the same way as in this work. Therefore, the addition of the extra stars in Fig. 4 guarantees a uniform comparison.

We observe that the stars with the lowest $\mathrm{Li}$ abundances ( $<1$ dex) show low $v \sin i$ values $\left(3-6 \mathrm{~km} \mathrm{~s}^{-1}\right.$ ) whereas the stars with the highest $\mathrm{Li}$, which presumably surround the dip, present a wide range of rotation rates $\left(\sim 4-10 \mathrm{~km} \mathrm{~s}^{-1}\right)$. This could be caused by the fact that the dip stars are older on average than the stars with higher $\mathrm{Li}$ at the same $v \sin i$ (upper panel of Fig. 4). There seems to be a slight increase of the upper envelope of $\mathrm{Li}$ abundances with rotation up to $10 \mathrm{~km} \mathrm{~s}^{-1}$, however, from that point $\mathrm{Li}$ abundances decrease sharply as the rotation increases. The fast rotators that form the upper envelope of Li abundances (within 10-30 $\mathrm{km} \mathrm{s}^{-1}$ ) have similar ages $(<1.5 \mathrm{Gyr})$ as the slower stars with high Li abundance. The lower panels of Fig. 4 illustrate that the metallicities and masses of both groups are comparable, thus we could expect that the higher rotation is producing extra depletion in these stars, as suggested by rotationally-induced mixing models. We could think then that, at young ages $(<1.5 \mathrm{Gyr})$, the $\mathrm{Li}$ dip is only formed by fast rotators, while for older ages the stars have had more time to deplete $\mathrm{Li}$ and to spin down at the same time, making it impossible to distinguish between rotationally induced mixing and other destruction mechanisms acting during the MS. 
Table 2. Li abundances for the fast rotators of Sect. 3.3.

\begin{tabular}{lccccccr}
\hline \hline Star & $\begin{array}{c}T_{\text {eff }} \\
(\mathrm{K})\end{array}$ & $\begin{array}{c}\log g \\
\left(\mathrm{~cm} \mathrm{~s}^{-2}\right)\end{array}$ & {$[\mathrm{Fe} / \mathrm{H}]$} & $\begin{array}{c}\text { Age } \\
(\mathrm{Gyr})\end{array}$ & $\begin{array}{c}\text { Mass } \\
\left(\mathrm{M}_{\odot}\right)\end{array}$ & $\begin{array}{c}v \sin i \\
\left(\mathrm{~km} \mathrm{~s}^{-1}\right)\end{array}$ & $A(\mathrm{Li})$ \\
\hline HD 142860 & 6361 & 4.07 & -0.09 & 2.89 & 1.21 & 10.65 & 2.32 \\
HD 89569 & 6469 & 4.08 & 0.09 & 2.14 & 1.41 & 11.33 & $<1.90$ \\
HD 86264 & 6300 & 4.06 & 0.25 & 2.19 & 1.39 & 12.55 & 1.50 \\
HD 210302 & 6405 & 4.24 & 0.10 & 1.50 & 1.29 & 13.68 & 2.42 \\
WASP-3 & 6423 & 4.42 & 0.04 & 0.93 & 1.27 & 15.21 & 2.60 \\
HD 30652 & 6494 & 4.29 & 0.04 & 0.84 & 1.29 & 17.01 & 2.25 \\
30AriB & 6284 & 4.35 & 0.12 & 2.55 & 1.32 & 42.61 & 2.72 \\
HAT-P-41 & 6479 & 4.39 & 0.13 & 1.05 & 1.35 & 20.11 & $<1.70$ \\
HAT-P-2 & 6414 & 4.18 & 0.04 & 1.91 & 1.30 & 20.50 & $<1.00$ \\
HAT-P-34 & 6509 & 4.24 & 0.08 & 1.17 & 1.35 & 24.08 & $<1.50$ \\
HD 8673 & 6472 & 4.27 & 0.14 & 0.55 & 1.24 & 26.91 & 1.50 \\
CoRoT-11 & 6343 & 4.27 & 0.04 & 1.58 & 1.28 & 36.72 & 2.10 \\
\hline
\end{tabular}

Notes. Stellar parameters from Tsantaki et al. (2014).

We checked whether the lack of stars with $v \sin i$ determination and Li upper limits could be biasing this effect. We could not determine $v \sin i$ values for nine stars (with Li upper limit), but only one out of those is young (WASP-32, 0.7 Gyr) and seems to be a slow rotator when we observe its spectrum. Thus, this is the only young star in our sample belonging to the dip with a low rotation rate. In any case, the small number of stars in this subsample suggests this result should be viewed with caution. Moreover, the rotational models predict a correlation between the rotation history of the star and Li depletion, rather than a correlation between the current rotation and the $\mathrm{Li}$ abundance (Pinsonneault 1997). There is another group of five fast rotators within the same $v \sin i$ range (10-30 $\mathrm{km} \mathrm{s}^{-1}$ ), forming a lower envelope for Li abundances, probably related to their greater ages. We note that these are the only stars with $\log g<4.2$ in Fig. 4 and we cannot rule out the possibility that they are subgiants. Curiously, for the two objects with the highest $v \sin i$ the trend changes, showing a higher Li abundance despite being older. We should consider this rise in $\mathrm{Li}$ abundance with caution since the determination of parameters becomes more difficult for the fastest rotators and the errors are three times larger than for the non-rotating counterparts.

\subsection{Li evolution: dependence on $[\mathrm{Fe} / \mathrm{H}]$ and age}

To extract information about the evolution of Li through the life of the galaxy, it is very common to evaluate its behaviour with the metallicity. The well-known "Spite plateau" shows how the abundances of $\mathrm{Li}$ are nearly constant at $[\mathrm{Fe} / \mathrm{H}] \lesssim-1$ dex while they increase as $[\mathrm{Fe} / \mathrm{H}]$ increases. However, the available studies of clusters and field stars do not include very metal-rich stars with the exception of the recently analyzed cluster NGC 6253 (Cummings et al. 2012) with $[\mathrm{Fe} / \mathrm{H}]=0.43$ dex. We note that a lower metallicity $([\mathrm{Fe} / \mathrm{H}]=0.23 \mathrm{dex})$ has been obtained for this cluster by other authors (Montalto et al. 2012). Our sample of metal-rich planet hosts represents a good opportunity to check how the $\mathrm{Li}$ abundances behave at $[\mathrm{Fe} / \mathrm{H}]>0.2$ dex.

In Fig. 5 we show the mean values of $\mathrm{Li}$, stellar mass, $T_{\text {eff }}$, and age for the six stars with the highest $\mathrm{Li}$ abundance in each bin of metallicity. We chose this number of stars per bin to compare our results with the values obtained by Lambert \& Reddy (2004) with a similar approach, who reported a good agreement with the maximum values found in open clusters of similar metallicity. We should consider the most metal-poor bins $([\mathrm{Fe} / \mathrm{H}]<-0.7 \mathrm{dex})$ with caution since we only have one or two

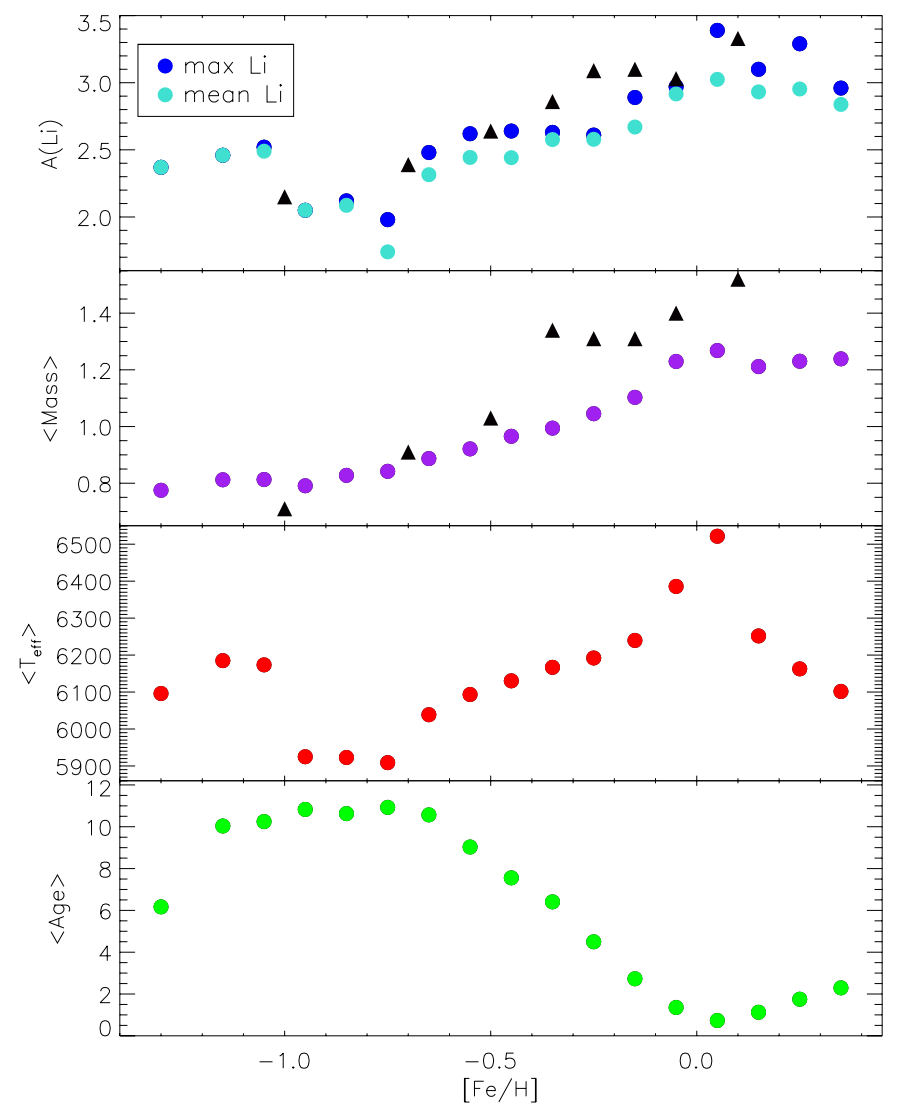

Fig. 5. Upper panel: maximum and mean values of Li in different metallicity bins for the six stars with the highest $\mathrm{Li}$ abundance in each metallicity bin (with $\log g>4.2$ ). The circles are the values from this work and the triangles denote the values from Lambert \& Reddy (2004). Remaining panels: mean values of mass, $T_{\text {eff }}$, and age for those six stars in each metallicity bin.

stars per bin and their temperatures fall out of the main trend. We compared our parameters with those derived by Casagrande et al. (2011) for these metal-poor stars and they agree well except for the most metal-poor star (HD 31128) for which Casagrande et al. (2011) gives a higher age, 8.26 Gyr.

For metallicities lower than solar, the Li abundance increases steadily with metallicity from a minimum at $[\mathrm{Fe} / \mathrm{H}]=-0.75 \mathrm{dex}$ with $A(\mathrm{Li}) \sim 2$, close to the "Spite plateau", up to $[\mathrm{Fe} / \mathrm{H}] \sim$ 0 dex. Why the abundances increase from there up to the solar 
metallicity? First, we can expect the more metallic stars to retain more of their initial Li because their masses are increasing (and thus the convective envelopes become shallower). Second, metal-rich stars are younger so they have less time to deplete their $\mathrm{Li}$ as observed in younger clusters (e.g. Sestito \& Randich 2005). Finally, the models of Li production point to an increase of Li with time in the interstellar medium (e.g. Fields \& Olive 1999; Prantzos 2012).

By contrast, for metallicities higher than solar, Li abundances seem to flatten and even decrease for the most metalrich stars. As expected, the age decreases as $[\mathrm{Fe} / \mathrm{H}]$ increases to reach a plateau for the most Li rich stars, where the age is between 1-2 Gyr. The maximum Li abundance, $A(\mathrm{Li})=3.39$, is found at solar metallicity, which coincides with the minimum in age. We note here that this value corresponds to WASP-66, a very young and hot $\operatorname{star}\left(T_{\mathrm{eff}}=7051 \mathrm{~K}\right)$ compared with the average $T_{\text {eff }}$ in our sample, hence the rise in average $T_{\text {eff }}$ shown in the third panel of Fig. 5. That value also matches the maximum Li abundances found in young clusters such as NGC 2264 (Sestito \& Randich 2005) and is very similar to the meteoritic abundance. Therefore it is possible that this represents the initial maximum $\mathrm{Li}$ abundance and those stars have not experienced any astration.

The standard model predicts that $\mathrm{Li}$ depletion is faster for more metallic stars since they have deeper convective zones. This is in contrast with the higher $\mathrm{Li}$ abundances found for the metal-rich stars. However, the models of Galactic Li production predict that initial $\mathrm{Li}$ abundance in a star becomes higher as the Galaxy evolves, i.e. as $[\mathrm{Fe} / \mathrm{H}]$ increases. Indeed, the high $\mathrm{Li}$ abundances found in meteorites or in young clusters require Galactic production to increase the primordial $\mathrm{Li}$ abundance (either $\sim 2.2$, from "Spite plateau" or $\sim 2.7$ from WMAP observations). The flattening observed at high $[\mathrm{Fe} / \mathrm{H}]$ is possibly a balance between the higher initial $\mathrm{Li}$ in those stars (due to a larger content of $\mathrm{Li}$ in the interstellar medium), and a stronger destruction of Li due to the deepening of stellar convective zones. This possibility is suggested by the models of Fields \& Olive (1999) who show that at super-solar metallicities the stellar Li depletion begins to affect the Li abundance in the inter-stellar medium, and thus flattening the correlation of initial $\mathrm{Li}$ and Fe. Therefore, in this scenario, we may think that the stars around solar metallicity represent the maximum $\mathrm{Li}$ in the Galaxy, which is similar to the initial value (and to the meteoritic value) since they are young and have not depleted it yet. As you move to super-solar metallicities the effect reverses and the high $[\mathrm{Fe} / \mathrm{H}]$ begins to produce Li depletion. However, we should also note, as shown in Fig. 5, that the $T_{\text {eff }}$ is also decreasing as $[\mathrm{Fe} / \mathrm{H}]$ increases, therefore the convective envelope is getting deeper ${ }^{4}$ and probably affecting the depletion of $\mathrm{Li}$.

The question remains how we can distinguish between a lower abundance of $\mathrm{Li}$ due to a lower initial abundance $([\mathrm{Fe} / \mathrm{H}]$ effect on Galactic production) or due to internal destruction during the MS ( $T_{\text {eff }}$ effect). In an attempt to disentangle both effects, we constructed samples of stars with different mass ranges but with $6000 \mathrm{~K}<T_{\text {eff }}<6200 \mathrm{~K}$, as shown in Fig. 6. We chose this $T_{\text {eff }}$ range because it is well populated with stars of different masses and metallicities. As expected, we cannot observe the stars with the highest $\mathrm{Li}$ abundance since we are not using the hottest stars. By restricting the sample, now the average $T_{\text {eff }}$, mass, and age of the stars are very similar in all the bins at

\footnotetext{
4 Pinsonneault et al. (2001) show that $T_{\text {eff }}$ is the main parameter determining the mass of convective envelopes with a very small effect of metallicity.
}

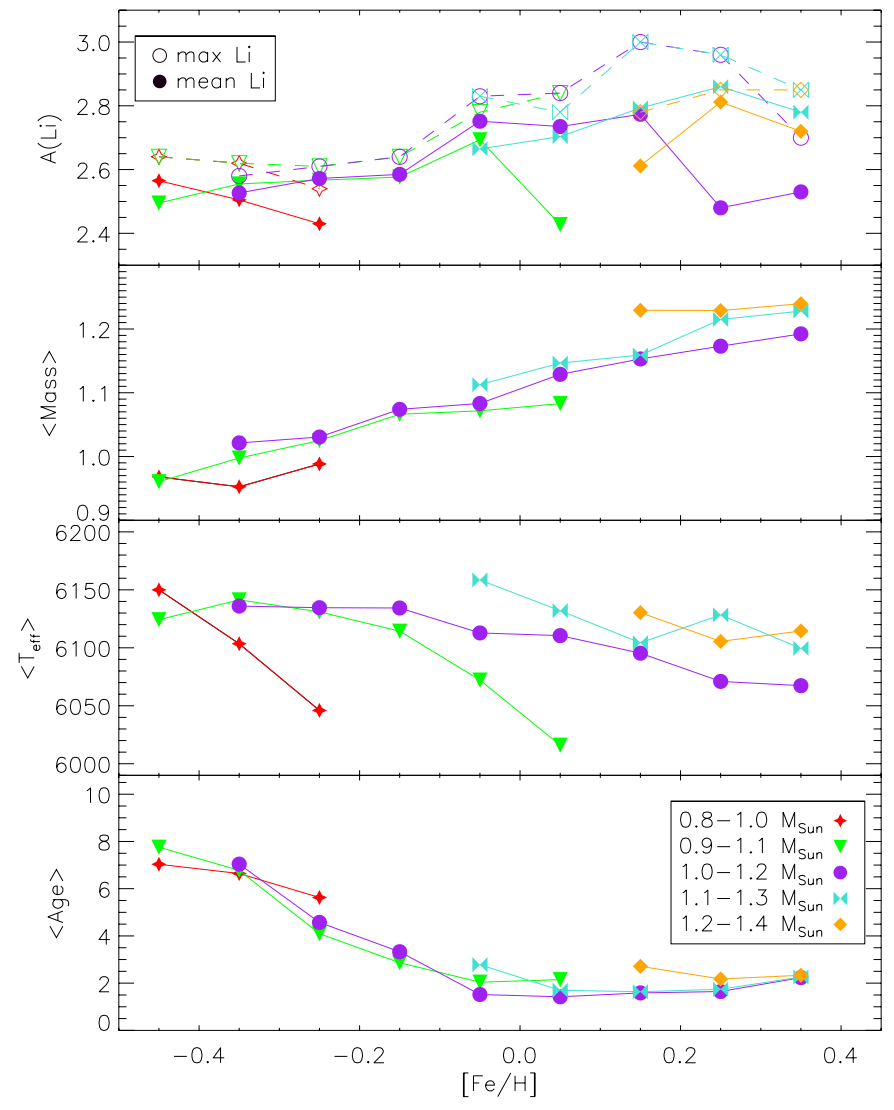

Fig. 6. Upper panel: maximum and mean values of Li in different metallicity bins for the six stars (when available) with the highest Li abundance in each metallicity bin (with $6000 \mathrm{~K}<T_{\text {eff }}<6200 \mathrm{~K}$ and $\log g>4.2$ ). Remaining panels: mean values of mass, $T_{\text {eff }}$, and age for those six stars in each metallicity bin.

super-solar metallicities, thus the observed variation of $\mathrm{Li}$ abundances should be triggered basically by the metallicity variation. We still observe the increase of $\mathrm{Li}$ with $[\mathrm{Fe} / \mathrm{H}]$, with a maximum at $[\mathrm{Fe} / \mathrm{H}]=0.15 \mathrm{dex}$ and a clear decrease for the subsamples between $1 M_{\odot}$ and $1.3 M_{\odot}$ (the most populated subsamples, designated by purple and blue symbols in Fig. 6). We evaluated the possible effect of planets on Li evolution, since as suggested before, planet hosts seem to have depleted more Li and our most metal-rich bins contain many of them. Thus, we reconstructed the samples between $1 M_{\odot}$ and $1.3 M_{\odot}$ with only comparison stars and we found that the behaviour is similar, i.e. Li decreases for the most metal-rich stars from $[\mathrm{Fe} / \mathrm{H}] \sim 0.15$ dex. Therefore, the lowest $\mathrm{Li}$ abundances found in the most metal-rich stars seem to be caused by a lower initial $\mathrm{Li}$ as predicted by some models of Galactic production (Fields \& Olive 1999).

It is commonly accepted that $\mathrm{Li}$ abundances decrease with age, though the main depletion takes place principally during very young ages and depends on initial rotation rates (e.g. Charbonnel \& Talon 2005) whereas after 1-2 Gyr the age effect is not so strong (Sestito \& Randich 2005). In Fig. 7 we show the $\mathrm{Li}$ abundance as a function of age in four different $T_{\text {eff }}$ bins. It is very clear that the higher abundances appear in the younger objects and then the Li upper envelope slightly decreases to reach a kind of a plateau. However we can still observe a high dispersion in Li for stars with similar $T_{\text {eff }}$, metallicity and age. For example, in the top-right panel, $(6000 \mathrm{~K}-6100 \mathrm{~K})$, for the most metallic stars $([\mathrm{Fe} / \mathrm{H}] \sim 0.4 \mathrm{dex}$, red symbols $)$ there is a dispersion of $\sim 0.8 \mathrm{dex}$ in $\mathrm{Li}$ abundance determinations (not considering 


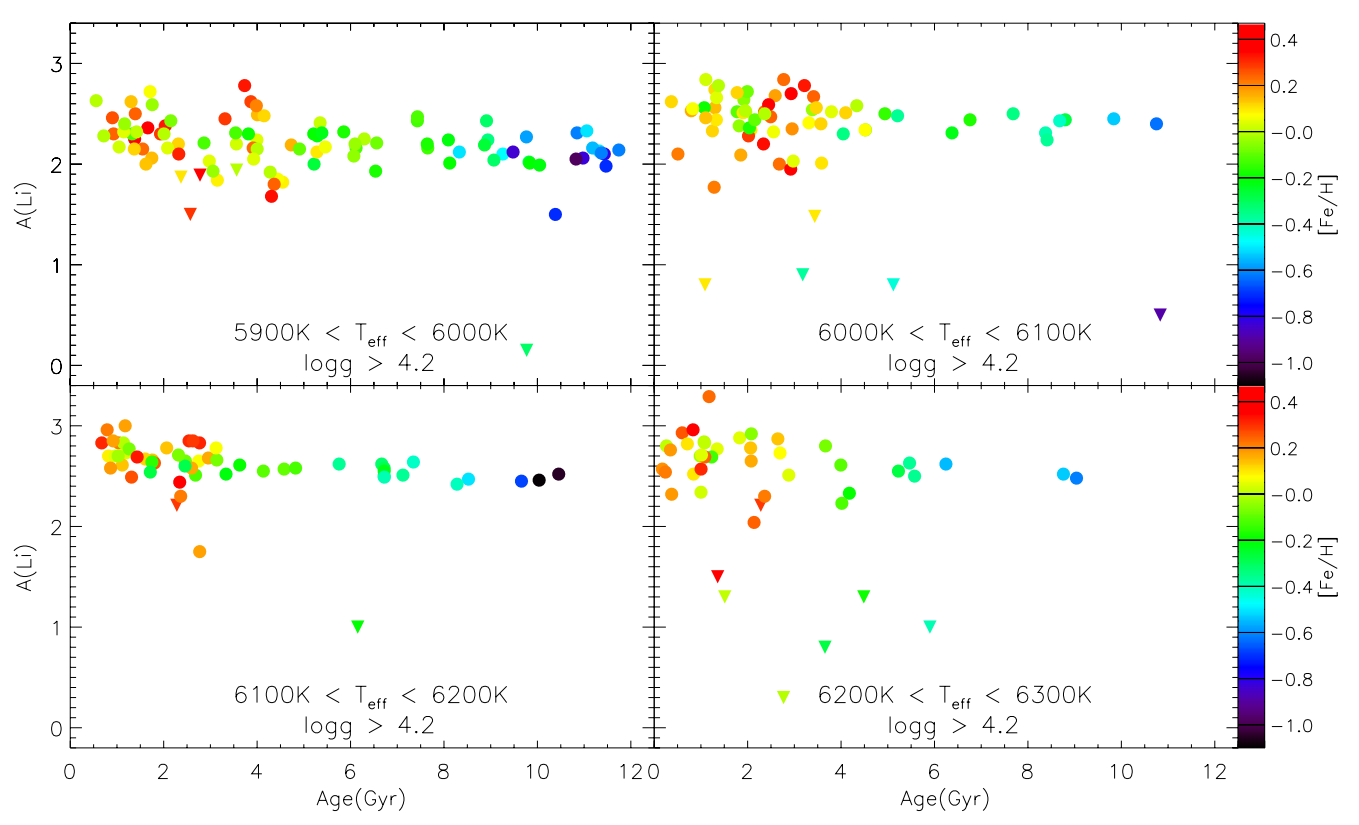

Fig. 7. Li abundances a function of age in several $T_{\text {eff }}$ regions. The metallicity values are shown with a colour scale.

upper limits). For stars of solar metallicity (green points) the dispersion reaches 0.4 dex for stars of similar age. We find a similar spread in other $T_{\text {eff }}$ and $[\mathrm{Fe} / \mathrm{H}]$ regions and the dispersion can reach values of 2 dex if we consider the upper limits. This fact reveals that an extra parameter is governing Li depletion. We may note here that when dealing with MS stars, the age determination is probably very uncertain (e.g. Jørgensen \& Lindegren 2005), at least significantly more uncertain than other stellar parameters determination. Therefore, it might be possible that these stars of apparently same age could have quite different ages and that could be the reason of the spread in abundances. For instance, in clusters like the Hyades or NGC 6243 the spread around $6000 \mathrm{~K}$ is quite small (Cummings et al. 2012). In contrast, M67 or Praesepe show a huge dispersion in Li abundances (Sestito \& Randich 2005).

\subsection{Li in the Galactic disks}

The recent work by Ramírez et al. (2012) presented a first attempt to compare Li abundances in the two Galactic disks. They found that the $\mathrm{Li}$ abundances for the thin disk stars (using a kinematical separation criteria) increase with metallicity, while for the thick disk stars the abundances have a nearly constant maximum value of $A(\mathrm{Li}) \sim 2.1 \mathrm{dex}$, similar to the "Spite plateau". However, once they cleaned their thin disk sample of the youngest and more massive stars, to allow for a less biased comparison with the older and cooler thick disk, they found a smoother transition from the thick to the thin disk.

In Fig. 8 we present a plot, similar to Fig. 5. in Ramírez et al. (2012), using only the stars of the HARPS samples analyzed in Adibekyan et al. (2012). We note that we have removed planet hosts for this section since they only represent $\sim 10 \%$ of the total sample and their abundances might be affected by the presence of planets (at least in the solar $T_{\text {eff }}$ region). To improve the comparison we added a set of cool stars $\left(T_{\text {eff }}<5600 \mathrm{~K}\right)$ belonging to the HARPS samples (see Table 6). We used both kinematic $^{5}$ and chemical criteria to separate the stellar populations

\footnotetext{
5 The kinematic separation was done using the prescription of Bensby et al. (2003) as presented in Adibekyan et al. (2012).
}

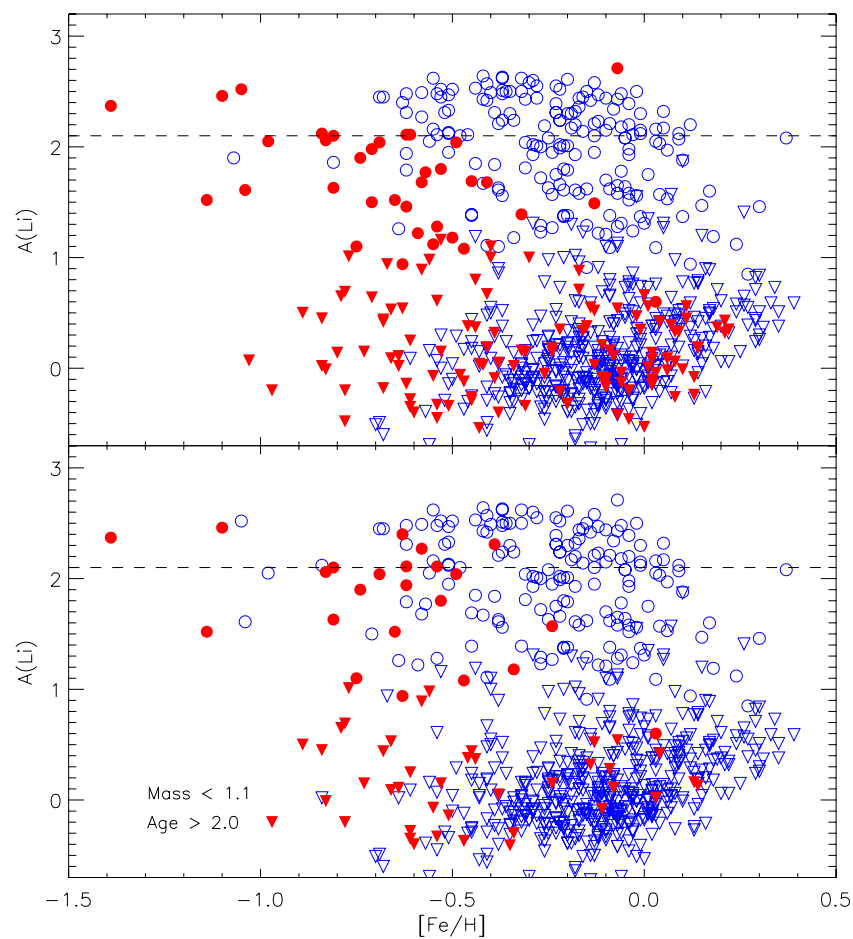

Fig. 8. Li abundances as a function of metallicity for the HARPS nonhost stars. Thin disk stars are depicted with blue symbols while thick disk stars are denoted with red symbols. Downward triangles are upper limits on Li. The separation of the Galactic populations are based on the abundances (top) and kinematics (bottom). The constraint on age is only applied for the thin disk stars, as in Ramírez et al. (2012).

(see Adibekyan et al. 2011, 2012, for details). We note that stars with $[\mathrm{Fe} / \mathrm{H}]>-0.2$ dex and showing enhancement in $\alpha$-element abundances were classified as members of a high- $\alpha$ metal-rich population in Adibekyan et al. $(2011,2013)$. Here we use the same symbol as for the thick disk stars to compare with the results of Ramírez et al. (2012) more easily.

Our thin disk stars also show slightly higher maximum abundances, decreasing for the more metal-rich stars as in the above 
mentioned work and reflecting the evolution of Li at high metallicities discussed in the previous section. However, our thick disk stars also show a decrease of $\mathrm{Li}$ with metallicity from $[\mathrm{Fe} / \mathrm{H}]>-0.5$ dex, whereas in Ramírez et al. (2012) the thick disk stars present a constant value close to the "Spite plateau" up to $[\mathrm{Fe} / \mathrm{H}] \sim-0.1$ dex. Furthermore, this decrease seems steeper for the thick disk stars than for the thin disk stars. The lack of Li-rich metal-rich thick disk stars in our sample when compared to that observed in Ramírez et al. (2012) can probably be explained by the different criteria used to separate the stellar populations. However, both our kinematic and chemical separation shows the same picture. We should note that our kinematic criteria suggest very few thick disk stars with $[\mathrm{Fe} / \mathrm{H}]>-0.3$ dex, while in the sample of Ramírez et al. (2012) this metallicity region is quite abundant of thick disk stars. A more detailed analysis of their metal-rich Li-rich thick disk stars is needed to understand the nature of these stars and the reason for the observed discrepancy.

\section{Summary}

We present new Li abundances for a total sample of 36 planet hosts and 229 stars without detected giant planets in the HARPS GTO samples, together with 88 additional extrasolar planet hosts from other sources. All these stars span over an effective temperature range $5900 \mathrm{~K}<T_{\text {eff }}<7200 \mathrm{~K}$. First, we find that planet hosts show an extra depletion of 0.07 dex in the $T_{\text {eff }}$ range $5900-6300 \mathrm{~K}$ as previously claimed by Gonzalez (2015). This offset is statistically significant but close to the average uncertainties of $\mathrm{Li}$ abundances. However, this offset seems to be stronger for stars hosting hot jupiters $(0.14 \mathrm{dex})$, something that could be explained by some models where the effect of planets on Li depletion is related with their mass and migration. This issue should be explored in the future with a larger sample of hot jupiters than the current sample of 24 stars analyzed here. In contrast, if we include the $\mathrm{Li}$ dependence on $v \sin i$ in our multivariate regression fit, the offset in $\mathrm{Li}$ abundance between the planet hosts and the comparison stars becomes positive but also decreases and becomes insignificant. This suggests that the difference in $v \sin i$ between both samples was causing the difference in Li abundances. Nevertheless, the number of stars for which we can derive $v \sin i$ is still too low to enable us to reach a conclusion regarding the effect of rotation on $\mathrm{Li}$ abundances for our sample of planet hosts.

We study the position of stellar mass of the Li dip at several metallicity bins. We confirm that the mass of the Li dip increases with the metallicity and extend this relation up to $[\mathrm{Fe} / \mathrm{H}]=$ 0.4 dex. However, the mass of all the stars in our sample increases with metallicity, thus reflecting the mass-metallicity relation for dwarfs of the same temperature and supporting the idea of a constant $T_{\text {eff }}$ for the Li dip. We also evaluate the behaviour of $\mathrm{Li}$ abundances with $v \sin i$ for the stars that surround the dip. We find that for the younger objects $(\$ 1.5 \mathrm{Gyr})$, a strong depletion of Li only happens for fast rotators $\left(\gtrsim 10 \mathrm{~km} \mathrm{~s}^{-1}\right)$, suggesting that the Li dip is formed due to rotationally induced mixing at early stages of the MS. However, for the older objects the Li dip is formed by slower rotators, making it impossible to differentiate between the previously mentioned mechanism (we do not know if those stars were fast rotators at younger ages) or other depletion processes taking place during the MS.

Finally we analyze the Li evolution with the metallicity (i.e. the age of the Galaxy) since our metal-rich sample represents a good opportunity to check the behaviour of $\mathrm{Li}$ at super-solar metallicities, which is not well studied in the literature. As expected from models of Galactic production of $\mathrm{Li}$, we observe an increase of $\mathrm{Li}$ abundances as the Galaxy evolves, i.e. as the metallicity increases. We find the maximum abundance around $[\mathrm{Fe} / \mathrm{H}] \sim 0.1 \mathrm{dex}$, with $A(\mathrm{Li})=3.39 \mathrm{dex}$, which is similar to the meteoritic value and the maximum $\mathrm{Li}$ abundances found in young clusters such as NGC 2264 (Sestito \& Randich 2005). That said, Li abundances flatten and even decrease for the most metal-rich stars. This is in agreement with the models of Fields \& Olive (1999) which suggest that the initial Li abundance of the most metal-rich stars is lower because the interstellar $\mathrm{Li}$ abundances have decreased due to the impact of stellar depletion during the evolution of the Galaxy. We also study the behaviour of $\mathrm{Li}$ in the context of thin and thick disks. We find a clear decrease of $\mathrm{Li}$ abundances at super-solar metallicities for the thin disk and a steeper decrease for thick disk stars that starts at $[\mathrm{Fe} / \mathrm{H}]>-0.5$ dex.

Acknowledgements. E.D.M., S.G.S. and V.Zh.A. acknowledge support from the Fundação para a Ciência e Tecnologia, FCT (Portugal) in the form of grants SFRH/BPD/76606/2011, SFRH/BPD/47611/2008, and SFRH/BPD/70574/2010, respectively. P.F. acknowledges support by Fundação para a Ciência e a Tecnologia (Portugal) through Investigador FCT contracts of reference IF/01037/2013 and POPH/FSE (EC) by FEDER funding through the program "Programa Operacional de Factores de Competitividade COMPETE". J.I.G.H. acknowledges support from the Spanish Ministry of Economy and Competitiveness (MINECO) under the 2011 Severo Ochoa Program MINECO SEV-2011-0187. N.C.S., A.M., and M.T. acknowledge support from the European Research Council/European Community under the FP7 through Starting Grant agreement number 239953, as well as support through programme Ciência 2007 funded by FCT/MCTES (Portugal) and POPH/FSE (EC), and in the form of grant PTDC/CTE-AST/098528/2008. A.M. is supported by the European Union Seventh Framework Programme (FP7/2007-2013) through grant agreement number 313014 (ETAEARTH). This research made use of the SIMBAD database operated at CDS, Strasbourg (France) and the Encyclopaedia of Extrasolar Planets. This work also made use of the IRAF facility.

\section{References}

Adibekyan, V. Z., Santos, N. C., Sousa, S. G., \& Israelian, G. 2011, A\&A, 535, L11

Adibekyan, V. Z., Sousa, S. G., Santos, N. C., et al. 2012, A\&A, 545, A32

Adibekyan, V. Z., Figueira, P., Santos, N. C., et al. 2013, A\&A, 554, A44

Alibert, Y., Mordasini, C., Benz, W., \& Winisdoerffer, C. 2005, A\&A, 434, 343 Ammler-von Eiff, M., Santos, N. C., Sousa, S. G., et al. 2009, A\&A, 507, 523

Anders, E., \& Grevesse, N. 1989, Geochim. Cosmochim. Acta, 53, 197

Balachandran, S. 1995, ApJ, 446, 203

Baumann, P., Ramírez, I., Meléndez, J., Asplund, M., \& Lind, K. 2010, A\&A 519, A87

Bensby, T., Feltzing, S., \& Lundström, I. 2003, A\&A, 410, 527

Boesgaard, A. M., \& Tripicco, M. J. 1986, ApJ, 302, L49

Bonomo, A. S., Hébrard, G., Santerne, A., et al. 2012, A\&A, 538, A96

Bouvier, J. 2008, A\&A, 489, L53

Casagrande, L., Schönrich, R., Asplund, M., et al. 2011, A\&A, 530, A138

Charbonnel, C., \& Talon, S. 2005, Science, 309, 2189

Chen, Y. Q., \& Zhao, G. 2006, AJ, 131, 1816

Chen, Y. Q., Nissen, P. E., Benoni, T., \& Zhao, G. 2001, A\&A, 371, 943

Cummings, J. D., Deliyannis, C. P., Anthony-Twarog, B., Twarog, B., \&

Maderak, R. M. 2012, AJ, 144, 137

da Silva, R., Udry, S., Bouchy, F., et al. 2007, A\&A, 473, 323

Delgado Mena, E., Israelian, G., González Hernández, J. I., et al. 2014, A\&A, 562, A92

Deliyannis, C. P., Demarque, P., \& Kawaler, S. D. 1990, ApJS, 73, 21

Fields, B. D., \& Olive, K. A. 1999, ApJ, 516, 797

Figueira, P., Faria, J. P., Delgado-Mena, E., et al. 2014, A\&A, 570, A21

François, P., Pasquini, L., Biazzo, K., Bonifacio, P., \& Palsa, R. 2013, A\&A, 552, A136

Garcia Lopez, R. J., Rebolo, R., \& Martin, E. L. 1994, A\&A, 282, 518

Ghezzi, L., Cunha, K., Smith, V. V., \& de la Reza, R. 2010, ApJ, 724, 154

Gonzalez, G. 2008, MNRAS, 386, 928

Gonzalez, G. 2014, MNRAS, 441, 1201 
Gonzalez, G. 2015, MNRAS, 446, 1020

Gonzalez, G., Carlson, M. K., \& Tobin, R. W. 2010, MNRAS, 403, 1368 Israelian, G., Santos, N. C., Mayor, M., \& Rebolo, R. 2004, A\&A, 414, 601 Israelian, G., Delgado Mena, E., Santos, N. C., et al. 2009, Nature, 462, 189 Jørgensen, B. R., \& Lindegren, L. 2005, A\&A, 436, 127

Lambert, D. L., \& Reddy, B. E. 2004, MNRAS, 349, 757

Lo Curto, G., Mayor, M., Benz, W., et al. 2010, A\&A, 512, A48

Luck, R. E., \& Heiter, U. 2006, AJ, 131, 3069

Mayor, M., Pepe, F., Queloz, D., et al. 2003, The Messenger, 114, 20

Molenda-Żakowicz, J., Sousa, S. G., Frasca, A., et al. 2013, MNRAS, 434, 1422

Montalto, M., Santos, N. C., Villanova, S., et al. 2012, MNRAS, 423, 3039

Mortier, A., Santos, N. C., Sousa, S. G., et al. 2013, A\&A, 558, A106

Mortier, A., Sousa, S. G., Adibekyan, V. Z., Brandão, I. M., \& Santos, N. C. 2014, A\&A, 572, A95

Moutou, C., Loeillet, B., Bouchy, F., et al. 2006, A\&A, 458, 327

Pinsonneault, M. 1997, ARA\&A, 35, 557

Pinsonneault, M. H., Kawaler, S. D., \& Demarque, P. 1990, ApJS, 74, 501

Pinsonneault, M. H., DePoy, D. L., \& Coffee, M. 2001, ApJ, 556, L59

Pont, F., Tamuz, O., Udalski, A., et al. 2008, A\&A, 487, 749

Prantzos, N. 2012, A\&A, 542, A67

Ramírez, I., Fish, J. R., Lambert, D. L., \& Allende Prieto, C. 2012, ApJ, 756, 46 Randich, S., Aharpour, N., Pallavicini, R., Prosser, C. F., \& Stauffer, J. R. 1997, A\&A, 323, 86

Richer, J., \& Michaud, G. 1993, ApJ, 416, 312
Ryan, S. G. 2000, MNRAS, 316, L35

Santos, N. C., Israelian, G., \& Mayor, M. 2004, A\&A, 415, 1153

Santos, N. C., Israelian, G., Mayor, M., et al. 2005, A\&A, 437, 1127

Santos, N. C., Ecuvillon, A., Israelian, G., et al. 2006, A\&A, 458, 997

Santos, N. C., Mayor, M., Bonfils, X., et al. 2011, A\&A, 526, A112

Santos, N. C., Sousa, S. G., Mortier, A., et al. 2013, A\&A, 556, A150

Schramm, D. N., Steigman, G., \& Dearborn, D. S. P. 1990, ApJ, 359, L55

Sestito, P., \& Randich, S. 2005, A\&A, 442, 615

Simón-Díaz, S., \& Herrero, A. 2014, A\&A, 562, A135

Soderblom, D. R., Jones, B. F., Balachandran, S., et al. 1993, AJ, 106, 1059

Somers, G., \& Pinsonneault, M. H. 2014 [arXiv: 1410.4238]

Sousa, S. G., Santos, N. C., Israelian, G., Mayor, M., \& Monteiro, M. J. P. F. G. 2006, A\&A, 458, 873

Sousa, S. G., Santos, N. C., Mayor, M., et al. 2008, A\&A, 487, 373

Sousa, S. G., Fernandes, J., Israelian, G., \& Santos, N. C. 2010, A\&A, 512, L5

Sousa, S. G., Santos, N. C., Israelian, G., et al. 2011a, A\&A, 526, A99

Sousa, S. G., Santos, N. C., Israelian, G., Mayor, M., \& Udry, S. 2011b, A\&A, 533, A141

Takeda, Y., \& Kawanomoto, S. 2005, PASJ, 57, 45

Takeda, Y., Honda, S., Kawanomoto, S., Ando, H., \& Sakurai, T. 2010, A\&A, 515, A93

Tsantaki, M., Sousa, S. G., Santos, N. C., et al. 2014, A\&A, 570, A80

Udalski, A., Pont, F., Naef, D., et al. 2008, A\&A, 482, 299

Zahn, J.-P. 1992, A\&A, 265, 115 
E. Delgado Mena et al.: Li abundances in F stars: planets, rotation, and Galactic evolution

Table 3. Li abundances for stars with planets from HARPS GTO samples.

\begin{tabular}{|c|c|c|c|c|c|c|c|c|c|c|}
\hline Star & $\begin{array}{l}T_{\text {eff }} \\
(\mathrm{K})\end{array}$ & $\begin{array}{c}\log g \\
\left(\mathrm{~cm} \mathrm{~s}^{-2}\right)\end{array}$ & $\begin{array}{c}\xi_{t} \\
\left(\mathrm{~km} \mathrm{~s}^{-1}\right)\end{array}$ & {$[\mathrm{Fe} / \mathrm{H}]$} & $\begin{array}{r}\text { Age } \\
\text { (Gyr) }\end{array}$ & $\begin{array}{l}\text { Mass } \\
\left(M_{\odot}\right)\end{array}$ & $A(\mathrm{Li})$ & Error & $\begin{array}{c}v \sin i \\
\left(\mathrm{~km} \mathrm{~s}^{-1}\right)\end{array}$ & Hot jupiter \\
\hline HD 142 & 6403 & 4.62 & 1.74 & 0.09 & 1.08 & 1.27 & 2.92 & 0.04 & 9.34 & no \\
\hline HD 10647 & 6218 & 4.62 & 1.22 & 0.00 & 0.26 & 1.16 & 2.80 & 0.03 & 5.30 & no \\
\hline HD 17051 & 6227 & 4.53 & 1.29 & 0.19 & 0.17 & 1.21 & 2.57 & 0.05 & 5.56 & no \\
\hline HD 19994 & 6289 & 4.48 & 1.72 & 0.24 & 2.14 & 1.34 & 2.04 & 0.07 & 8.15 & no \\
\hline HD 23079 & 5980 & 4.48 & 1.12 & -0.12 & 6.12 & 1.00 & 2.16 & 0.04 & 2.96 & no \\
\hline HD 39091 & 6003 & 4.42 & 1.12 & 0.09 & 2.07 & 1.11 & 2.34 & 0.04 & 2.96 & no \\
\hline HD 52265 & 6136 & 4.36 & 1.32 & 0.21 & 1.04 & 1.20 & 2.83 & 0.05 & - & no \\
\hline HD 75289 & 6161 & 4.37 & 1.29 & 0.30 & 0.68 & 1.21 & 2.83 & 0.04 & 4.30 & no \\
\hline HD 82943 & 5989 & 4.43 & 1.10 & 0.26 & 0.90 & 1.15 & 2.46 & 0.04 & 2.75 & no \\
\hline HD 108147 & 6260 & 4.47 & 1.30 & 0.18 & 0.37 & 1.23 & 2.32 & 0.03 & 5.85 & no \\
\hline HD 117618 & 5990 & 4.41 & 1.13 & 0.03 & 4.00 & 1.08 & 2.24 & 0.03 & 3.67 & no \\
\hline HD 121504 & 6022 & 4.49 & 1.12 & 0.14 & 1.30 & 1.14 & 2.56 & 0.03 & 3.61 & no \\
\hline HD 169830 & 6361 & 4.21 & 1.56 & 0.18 & 2.04 & 1.39 & $<1.10$ & - & 3.49 & no \\
\hline HD 179949 & 6287 & 4.54 & 1.36 & 0.21 & 0.24 & 1.24 & 2.54 & 0.04 & 6.84 & yes \\
\hline HD 196050 & 5917 & 4.32 & 1.21 & 0.23 & 3.91 & 1.12 & 2.16 & 0.03 & 3.34 & no \\
\hline HD 208487 & 6146 & 4.48 & 1.24 & 0.08 & 0.82 & 1.17 & 2.70 & 0.04 & 4.01 & no \\
\hline HD 209458 & 6118 & 4.50 & 1.21 & 0.03 & 1.19 & 1.13 & 2.73 & 0.05 & - & yes \\
\hline HD 212301 & 6271 & 4.55 & 1.29 & 0.18 & 0.35 & 1.24 & 2.76 & 0.04 & 5.76 & yes \\
\hline HD 213240 & 5982 & 4.27 & 1.25 & 0.14 & 4.01 & 1.19 & 2.49 & 0.05 & 3.50 & no \\
\hline HD 216435 & 6008 & 4.20 & 1.34 & 0.24 & 3.41 & 1.28 & 2.67 & 0.04 & 5.13 & no \\
\hline HD 221287 & 6374 & 4.62 & 1.29 & 0.04 & 0.33 & 1.22 & 2.97 & 0.04 & 4.77 & no \\
\hline HD 7449 & 6024 & 4.51 & 1.11 & -0.11 & 1.77 & 1.06 & 2.52 & 0.03 & 3.51 & no \\
\hline HD 10180 & 5911 & 4.39 & 1.11 & 0.08 & 4.55 & 1.06 & 1.82 & 0.03 & 2.80 & no \\
\hline HD 93385 & 5977 & 4.42 & 1.14 & 0.02 & 3.56 & 1.07 & 2.20 & 0.03 & 2.99 & no \\
\hline HD 134060 & 5966 & 4.43 & 1.10 & 0.14 & 1.75 & 1.12 & 2.06 & 0.04 & 3.21 & no \\
\hline \multicolumn{11}{|l|}{ HARPS-4 } \\
\hline HD 190984 & 6007 & 4.02 & 1.58 & -0.49 & 4.60 & 1.16 & $<0.50$ & - & 3.23 & no \\
\hline \multicolumn{11}{|l|}{ HARPS-2 } \\
\hline HD 125612 & 5913 & 4.43 & 1.02 & 0.24 & 1.39 & 1.10 & 2.50 & 0.05 & - & no \\
\hline HD 145377 & 6054 & 4.53 & 1.11 & 0.12 & 1.25 & 1.12 & 2.33 & 0.04 & 3.76 & no \\
\hline HD 148156 & 6251 & 4.51 & 1.36 & 0.25 & 0.60 & 1.21 & 2.93 & 0.02 & 5.41 & no \\
\hline HD 153950 & 6074 & 4.39 & 1.23 & -0.01 & 4.34 & 1.12 & 2.58 & 0.04 & 3.41 & no \\
\hline HD 156411 & 5910 & 3.99 & 1.31 & -0.11 & 4.21 & 1.25 & $<0.30$ & - & 3.34 & no \\
\hline HD 217786 & 5966 & 4.35 & 1.12 & -0.14 & 7.65 & 1.02 & 2.16 & 0.07 & - & no \\
\hline HD 25171 & 6160 & 4.43 & 1.22 & -0.11 & 4.13 & 1.09 & 2.55 & 0.06 & - & no \\
\hline HD 72659 & 5926 & 4.24 & 1.13 & -0.02 & 6.29 & 1.06 & 2.25 & 0.07 & - & no \\
\hline HD 8535 & 6158 & 4.42 & 1.25 & 0.04 & 1.76 & 1.15 & 2.65 & 0.03 & 3.07 & no \\
\hline HD 9578 & 6055 & 4.52 & 1.07 & 0.11 & 1.30 & 1.12 & 2.74 & 0.04 & 2.36 & no \\
\hline
\end{tabular}

Notes. Parameters from Sousa et al. (2008, 2011a,b).

Table 4. Li abundances for stars without detected planets from HARPS GTO samples.

\begin{tabular}{lccccccccc}
\hline \hline Star & $\begin{array}{c}T_{\text {eff }} \\
(\mathrm{K})\end{array}$ & $\begin{array}{c}\log g \\
\left(\mathrm{~cm} \mathrm{~s}^{-2}\right)\end{array}$ & $\begin{array}{c}\xi_{t} \\
\left(\mathrm{~km} \mathrm{~s}^{-1}\right)\end{array}$ & {$[\mathrm{Fe} / \mathrm{H}]$} & $\begin{array}{c}\text { Age } \\
(\mathrm{Gyr})\end{array}$ & $\begin{array}{c}\text { Mass } \\
\left(M_{\odot}\right)\end{array}$ & $A(\mathrm{Li})$ & Error & $\begin{array}{c}v \sin i \\
\left(\mathrm{~km} \mathrm{~s}^{-1}\right)\end{array}$ \\
\hline HD 361 & 5913 & 4.60 & 1.00 & -0.12 & 1.33 & 1.03 & 2.30 & 0.05 & 2.96 \\
HD 1388 & 5954 & 4.41 & 1.13 & -0.01 & 4.00 & 1.04 & 2.15 & 0.03 & 3.18 \\
HD 1581 & 5977 & 4.51 & 1.12 & -0.18 & 5.86 & 0.99 & 2.32 & 0.02 & 3.03 \\
HD 3823 & 6022 & 4.31 & 1.39 & -0.28 & 8.80 & 0.99 & 2.44 & 0.03 & 3.00 \\
HD 6735 & 6082 & 4.49 & 1.15 & -0.06 & 1.92 & 1.09 & 2.64 & 0.04 & 3.71 \\
HD 7134 & 5940 & 4.41 & 1.17 & -0.29 & 9.82 & 0.92 & 2.02 & 0.05 & 2.71 \\
HD 9782 & 6023 & 4.42 & 1.09 & 0.09 & 1.33 & 1.12 & 2.44 & 0.03 & 3.06 \\
HD 11226 & 6098 & 4.35 & 1.28 & 0.04 & 3.79 & 1.14 & 2.52 & 0.03 & 3.03 \\
HD 23456 & 6178 & 4.56 & 1.38 & -0.32 & 6.67 & 0.99 & 2.62 & 0.04 & 3.20 \\
HD 31822 & 6042 & 4.57 & 1.15 & -0.19 & 1.07 & 1.04 & 2.56 & 0.04 & 3.77 \\
HD 36108 & 5916 & 4.33 & 1.21 & -0.21 & 9.84 & 0.97 & 2.01 & 0.05 & 2.95 \\
HD 36379 & 6030 & 4.30 & 1.29 & -0.17 & 6.76 & 1.07 & 2.44 & 0.04 & 2.98 \\
HD 38382 & 6082 & 4.45 & 1.18 & 0.03 & 1.34 & 1.12 & 2.66 & 0.05 & 3.29 \\
HD 38973 & 6016 & 4.42 & 1.14 & 0.05 & 2.56 & 1.10 & 2.32 & 0.04 & 3.29 \\
HD 44120 & 6052 & 4.25 & 1.31 & 0.12 & 3.47 & 1.23 & 2.56 & 0.04 & 3.39 \\
HD 44447 & 5999 & 4.37 & 1.26 & -0.22 & 8.10 & 1.00 & 2.24 & 0.04 & 2.84 \\
\hline
\end{tabular}

Notes. Parameters from Sousa et al. (2008, 2011a,b). 
Table 4. continued.

\begin{tabular}{|c|c|c|c|c|c|c|c|c|c|}
\hline Star & $\begin{array}{l}T_{\text {eff }} \\
(\mathrm{K}) \\
\end{array}$ & $\begin{array}{c}\log g \\
\left(\mathrm{~cm} \mathrm{~s}^{-2}\right)\end{array}$ & $\begin{array}{c}\begin{array}{c}\xi_{t} \\
\left(\mathrm{~km} \mathrm{~s}^{-1}\right)\end{array} \\
\end{array}$ & {$[\mathrm{Fe} / \mathrm{H}]$} & $\begin{array}{r}\text { Age } \\
(\mathrm{Gyr}) \\
\end{array}$ & $\begin{array}{l}\text { Mass } \\
\left(M_{\odot}\right) \\
\end{array}$ & $A(\mathrm{Li})$ & Error & $\begin{array}{c}v \sin i \\
\left(\mathrm{~km} \mathrm{~s}^{-1}\right) \\
\end{array}$ \\
\hline HD 55693 & 5914 & 4.43 & 1.07 & 0.29 & 2.57 & 1.11 & $<1.50$ & - & 3.38 \\
\hline HD 65907A & 5945 & 4.52 & 1.05 & -0.31 & 9.77 & 0.91 & $<0.15$ & - & 2.93 \\
\hline HD 68978A & 5965 & 4.48 & 1.09 & 0.04 & 1.14 & 1.09 & 2.32 & 0.04 & 3.04 \\
\hline HD 69655 & 5961 & 4.44 & 1.15 & -0.18 & 7.64 & 0.97 & 2.20 & 0.03 & 2.71 \\
\hline HD 70889 & 6051 & 4.49 & 1.13 & 0.11 & 0.37 & 1.14 & 2.62 & 0.03 & 3.45 \\
\hline HD 71479 & 6026 & 4.42 & 1.19 & 0.24 & 2.49 & 1.16 & 2.47 & 0.03 & 2.98 \\
\hline HD 73121 & 6091 & 4.30 & 1.34 & 0.09 & 3.44 & 1.22 & $<1.48$ & - & 3.49 \\
\hline HD 73524 & 6017 & 4.43 & 1.14 & 0.16 & 0.79 & 1.15 & 2.53 & 0.03 & 3.15 \\
\hline HD 83529 & 5902 & 4.35 & 1.11 & -0.22 & 10.05 & 0.94 & 1.99 & 0.04 & 2.47 \\
\hline HD 88742 & 5981 & 4.52 & 1.07 & -0.02 & 0.72 & 1.09 & 2.28 & 0.03 & 2.45 \\
\hline HD 95456 & 6276 & 4.35 & 1.40 & 0.16 & 2.08 & 1.27 & 2.65 & 0.02 & 3.28 \\
\hline HD 105837 & 5907 & 4.54 & 1.14 & -0.51 & 11.32 & 0.84 & 2.13 & 0.04 & 2.92 \\
\hline HD 119638 & 6069 & 4.42 & 1.22 & -0.15 & 4.94 & 1.03 & 2.50 & 0.04 & 2.72 \\
\hline HD 122862 & 5982 & 4.23 & 1.29 & -0.12 & 7.43 & 1.06 & 2.47 & 0.03 & 2.71 \\
\hline HD 125881 & 6036 & 4.49 & 1.10 & 0.06 & 0.82 & 1.12 & 2.55 & 0.03 & 2.94 \\
\hline HD 144585 & 5914 & 4.35 & 1.15 & 0.33 & 4.31 & 1.14 & 1.68 & 0.05 & 2.87 \\
\hline HD 145666 & 5958 & 4.53 & 1.04 & -0.04 & 1.16 & 1.07 & 2.40 & 0.03 & 2.75 \\
\hline HD 157338 & 6027 & 4.44 & 1.17 & -0.08 & 4.52 & 1.04 & 2.34 & 0.03 & 2.83 \\
\hline HD 162396 & 6090 & 4.27 & 1.43 & -0.35 & 7.68 & 1.01 & 2.50 & 0.03 & 2.84 \\
\hline HD 168871 & 5983 & 4.42 & 1.17 & -0.09 & 6.56 & 1.03 & 2.21 & 0.03 & 3.14 \\
\hline HD 171990 & 6045 & 4.14 & 1.40 & 0.06 & 3.47 & 1.29 & 2.77 & 0.02 & 3.06 \\
\hline HD 180409 & 6013 & 4.52 & 1.16 & -0.17 & 2.04 & 1.03 & 2.36 & 0.05 & 3.43 \\
\hline HD 193193 & 5979 & 4.40 & 1.15 & -0.05 & 6.07 & 1.06 & 2.08 & 0.03 & 3.19 \\
\hline HD 196800 & 6010 & 4.37 & 1.17 & 0.19 & 2.95 & 1.15 & 2.35 & 0.05 & 3.54 \\
\hline HD 199190 & 5926 & 4.26 & 1.14 & 0.15 & 4.73 & 1.12 & 2.19 & 0.03 & 2.69 \\
\hline HD 199960 & 5973 & 4.39 & 1.13 & 0.28 & 3.31 & 1.14 & 2.45 & 0.04 & 3.30 \\
\hline HD 204385 & 6033 & 4.44 & 1.15 & 0.07 & 3.31 & 1.11 & 2.41 & 0.04 & 3.63 \\
\hline HD 207129 & 5937 & 4.49 & 1.06 & 0.00 & 1.42 & 1.07 & 2.32 & 0.03 & 2.71 \\
\hline HD 220367 & 6128 & 4.37 & 1.34 & -0.21 & 6.15 & 1.08 & $<1.00$ & - & 3.48 \\
\hline HD 221356 & 6112 & 4.53 & 1.12 & -0.20 & 3.63 & 1.04 & 2.61 & 0.03 & 3.69 \\
\hline \multicolumn{10}{|l|}{ HARPS-4 } \\
\hline HD 25704 & 5942 & 4.52 & 1.37 & -0.83 & 10.98 & 0.81 & 2.06 & 0.05 & 3.12 \\
\hline HD 31128 & 6096 & 4.90 & 3.02 & -1.39 & 6.17 & 0.77 & 2.37 & 0.08 & - \\
\hline HD 38510 & 5914 & 4.32 & 1.30 & -0.81 & 11.43 & 0.86 & 2.10 & 0.07 & - \\
\hline HD 59984 & 5962 & 4.18 & 1.45 & -0.69 & 10.42 & 0.89 & 2.45 & 0.03 & 2.67 \\
\hline HD 61902 & 6209 & 4.38 & 1.58 & -0.62 & 9.04 & 0.93 & 2.48 & 0.05 & 4.26 \\
\hline HD 68284 & 5933 & 4.08 & 1.40 & -0.50 & 8.00 & 1.01 & 2.52 & 0.04 & 3.37 \\
\hline HD 88474 & 6122 & 3.91 & 1.91 & -0.48 & 3.60 & 1.23 & 3.11 & 0.05 & - \\
\hline HD 90422 & 6085 & 4.14 & 1.67 & -0.62 & 7.86 & 0.99 & 1.79 & 0.10 & 3.86 \\
\hline HD 94444 & 5998 & 4.34 & 1.29 & -0.62 & 10.85 & 0.87 & 2.31 & 0.07 & - \\
\hline HD 95860 & 6054 & 4.48 & 1.25 & -0.31 & - & - & 2.25 & 0.07 & - \\
\hline HD 102200 & 6185 & 4.59 & 1.52 & -1.10 & 10.04 & 0.81 & 2.46 & 0.05 & - \\
\hline HD 109310 & 5922 & 4.55 & 1.15 & -0.51 & 8.33 & 0.87 & 2.12 & 0.05 & 2.95 \\
\hline HD 109684 & 5992 & 4.38 & 1.22 & -0.34 & 8.94 & 0.93 & 2.24 & 0.10 & 3.04 \\
\hline HD 119949 & 6359 & 4.47 & 1.65 & -0.41 & 5.05 & 1.07 & 2.57 & 0.08 & - \\
\hline HD 123517 & 6082 & 4.08 & 1.53 & 0.09 & - & - & 1.94 & 0.10 & 5.81 \\
\hline HD 128340 & 6259 & 4.64 & 1.42 & -0.55 & 6.24 & 0.95 & 2.62 & 0.07 & - \\
\hline HD 144589 & 6372 & 4.28 & 1.72 & -0.05 & - & - & 1.82 & 0.10 & - \\
\hline HD 145344 & 6143 & 4.39 & 1.48 & -0.68 & 9.66 & 0.90 & 2.45 & 0.06 & - \\
\hline HD 148816 & 5908 & 4.39 & 1.36 & -0.71 & 11.47 & 0.86 & 1.98 & 0.07 & 2.70 \\
\hline HD 150177 & 6216 & 4.18 & 1.76 & -0.58 & 7.29 & 1.02 & 2.49 & 0.02 & 3.84 \\
\hline HD 195633 & 6154 & 4.25 & 1.47 & -0.51 & 8.52 & 0.98 & 2.47 & 0.06 & 3.95 \\
\hline HD 196892 & 6072 & 4.50 & 1.21 & -0.89 & 10.83 & 0.83 & $<0.50$ & - & 2.23 \\
\hline HD 197536 & 6105 & 4.39 & 1.34 & -0.41 & 8.28 & 0.96 & 2.42 & 0.04 & - \\
\hline HD 210752 & 5951 & 4.53 & 1.20 & -0.58 & 9.77 & 0.86 & 2.27 & 0.03 & - \\
\hline HD 215257 & 6052 & 4.46 & 1.40 & -0.63 & 10.75 & 0.87 & 2.40 & 0.04 & 2.80 \\
\hline HD 218504 & 5962 & 4.34 & 1.21 & -0.55 & 11.18 & 0.90 & 2.16 & 0.03 & 3.00 \\
\hline HD 223854 & 6080 & 4.08 & 1.60 & -0.54 & 8.43 & 0.99 & 2.48 & 0.05 & - \\
\hline HD 224347 & 6092 & 4.27 & 1.31 & -0.42 & 8.68 & 0.95 & 2.43 & 0.05 & - \\
\hline $\mathrm{BD}-084501$ & 6216 & 4.81 & 2.36 & -1.38 & - & - & 2.54 & 0.15 & - \\
\hline CD -231087 & 6788 & 4.67 & 1.82 & -0.24 & - & - & $<1.00$ & - & 5.37 \\
\hline CD -436810 & 6011 & 4.41 & 1.09 & -0.44 & 5.12 & 0.91 & $<0.80$ & - & 3.85 \\
\hline$C D-451246$ & 5960 & 4.42 & 0.75 & -0.86 & - & - & 2.17 & 0.10 & - \\
\hline CD -571633 & 5975 & 4.46 & 1.14 & -0.85 & - & - & 2.27 & 0.08 & - \\
\hline
\end{tabular}


Table 4. continued.

\begin{tabular}{|c|c|c|c|c|c|c|c|c|c|}
\hline Star & $\begin{array}{l}T_{\text {eff }} \\
(\mathrm{K})\end{array}$ & $\begin{array}{c}\log g \\
\left(\mathrm{~cm} \mathrm{~s}^{-2}\right)\end{array}$ & $\begin{array}{c}\xi_{t} \\
\left(\mathrm{~km} \mathrm{~s}^{-1}\right)\end{array}$ & {$[\mathrm{Fe} / \mathrm{H}]$} & $\begin{array}{l}\text { Age } \\
(\mathrm{Gyr})\end{array}$ & $\begin{array}{l}\text { Mass } \\
\left(M_{\odot}\right)\end{array}$ & $A(\mathrm{Li})$ & Error & $\begin{array}{c}v \sin i \\
\left(\mathrm{~km} \mathrm{~s}^{-1}\right)\end{array}$ \\
\hline \multicolumn{10}{|l|}{ HARPS-2 } \\
\hline HD 101612 & 6281 & 4.41 & 1.17 & -0.36 & 5.57 & 1.03 & 2.50 & 0.08 & - \\
\hline HD 102300 & 5987 & 4.23 & 1.14 & -0.31 & 8.91 & 0.97 & 2.43 & 0.08 & - \\
\hline HD 103891 & 6072 & 4.05 & 1.50 & -0.19 & 3.71 & 1.27 & $<0.10$ & - & 3.69 \\
\hline HD 104760 & 5953 & 4.43 & 1.02 & 0.12 & 2.32 & 1.10 & 2.20 & 0.05 & - \\
\hline HD 105938 & 6208 & 4.27 & 1.60 & 0.03 & 2.88 & 1.34 & 2.51 & 0.06 & - \\
\hline HD 106290 & 6012 & 4.55 & 1.03 & 0.13 & 1.10 & 1.10 & 2.46 & 0.06 & - \\
\hline HD 108063 & 6081 & 4.11 & 1.54 & 0.55 & 2.06 & 1.58 & $<0.70$ & - & 4.60 \\
\hline HD 111564 & 6004 & 4.37 & 1.13 & 0.07 & 4.51 & 1.12 & 2.34 & 0.07 & - \\
\hline HD 112283 & 6433 & 4.84 & 1.86 & -0.13 & 0.94 & 1.15 & 2.89 & 0.08 & - \\
\hline HD 115341 & 6058 & 4.55 & 1.10 & -0.01 & 1.95 & 1.09 & 2.53 & 0.06 & - \\
\hline HD 115773 & 6312 & 4.23 & 1.57 & -0.08 & 2.99 & 1.32 & $<0.45$ & - & 4.67 \\
\hline HD 116410 & 5939 & 4.43 & 1.07 & 0.23 & 1.55 & 1.11 & 2.15 & 0.05 & - \\
\hline HD 119629 & 6250 & 4.17 & 1.73 & -0.17 & 3.25 & 1.30 & $<1.30$ & - & 4.31 \\
\hline HD 123619 & 6166 & 4.45 & 1.38 & -0.32 & 6.72 & 1.01 & 2.50 & 0.08 & - \\
\hline HD 123651 & 5926 & 4.55 & 1.05 & -0.48 & 9.26 & 0.88 & 2.10 & 0.10 & - \\
\hline HD 126793 & 5910 & 4.46 & 1.26 & -0.71 & 10.39 & 0.82 & 1.50 & 0.15 & - \\
\hline HD 128571 & 6159 & 4.40 & 1.23 & -0.37 & 7.12 & 0.98 & 2.51 & 0.10 & - \\
\hline HD 129829 & 6196 & 4.66 & 1.30 & -0.16 & 1.75 & 1.08 & 2.64 & 0.07 & - \\
\hline HD 130989 & 6414 & 4.27 & 1.85 & -0.23 & 3.21 & 1.28 & $<1.20$ & - & - \\
\hline HD 135468 & 6417 & 4.25 & 1.82 & -0.02 & 2.17 & 1.45 & 2.86 & 0.07 & 5.94 \\
\hline HD 135625 & 6003 & 4.32 & 1.16 & 0.12 & 4.10 & 1.16 & 2.51 & 0.04 & - \\
\hline HD 139590 & 6200 & 4.49 & 1.31 & 0.13 & 2.06 & 1.22 & 2.78 & 0.05 & - \\
\hline HD 139879 & 6203 & 4.61 & 1.25 & 0.30 & 1.00 & 1.22 & 2.57 & 0.08 & - \\
\hline HD 141128 & 6758 & 4.67 & 1.65 & 0.07 & 0.32 & 1.34 & $<2.00$ & - & - \\
\hline HD 141597 & 6285 & 4.38 & 1.23 & -0.40 & 5.90 & 1.03 & $<1.00$ & - & - \\
\hline HD 143638 & 5954 & 4.48 & 1.04 & -0.27 & 5.22 & 0.95 & 2.00 & 0.08 & 2.84 \\
\hline HD 143790 & 6557 & 4.11 & 2.05 & -0.06 & 1.80 & 1.56 & 2.90 & 0.06 & 5.43 \\
\hline HD 144846 & 6102 & 4.52 & 1.11 & 0.13 & 1.11 & 1.14 & 2.61 & 0.06 & - \\
\hline HD 144880 & 6152 & 4.38 & 1.33 & -0.30 & 6.72 & 1.04 & 2.58 & 0.06 & - \\
\hline HD 14745 & 6290 & 4.72 & 1.46 & -0.14 & 1.23 & 1.11 & 2.69 & 0.05 & - \\
\hline HD 148211 & 5922 & 4.34 & 1.31 & -0.62 & 11.37 & 0.87 & 2.11 & 0.04 & 2.73 \\
\hline HD 149200 & 6416 & 4.64 & 1.74 & 0.15 & 0.96 & 1.29 & 2.45 & 0.09 & - \\
\hline HD 150139 & 5968 & 4.29 & 1.29 & -0.51 & 11.06 & 0.91 & 2.33 & 0.04 & - \\
\hline HD 151772 & 6631 & 4.81 & 2.27 & -0.36 & 3.02 & 1.13 & 2.22 & 0.15 & - \\
\hline HD 152433 & 6144 & 4.49 & 1.19 & -0.10 & 4.58 & 1.11 & 2.57 & 0.05 & - \\
\hline HD 153276 & 6000 & 4.48 & 1.12 & 0.04 & 2.98 & 1.09 & 2.03 & 0.10 & - \\
\hline HD 154195 & 5961 & 4.48 & 1.01 & -0.19 & 3.82 & 0.99 & 2.30 & 0.08 & - \\
\hline HD 156098 & 6517 & 4.20 & 2.06 & 0.18 & 1.44 & 1.70 & $<0.80$ & - & 6.30 \\
\hline HD 156991 & 5934 & 4.57 & 0.99 & -0.07 & 2.15 & 1.01 & 2.43 & 0.06 & - \\
\hline HD 160089 & 6312 & 4.78 & 1.60 & 0.11 & 0.67 & 1.20 & 2.87 & 0.08 & - \\
\hline HD 161566 & 6230 & 4.20 & 1.67 & -0.28 & 3.66 & 1.25 & $<0.80$ & - & 3.87 \\
\hline HD 163102 & 6433 & 4.64 & 1.94 & 0.00 & 1.13 & 1.24 & 2.94 & 0.06 & - \\
\hline HD 16382 & 5953 & 4.50 & 1.04 & 0.03 & 3.93 & 1.06 & 2.05 & 0.10 & - \\
\hline HD 173885 & 6264 & 4.37 & 1.61 & -0.20 & 4.18 & 1.21 & 2.33 & 0.08 & - \\
\hline HD 174153 & 6196 & 4.49 & 1.34 & -0.08 & 3.14 & 1.12 & 2.66 & 0.05 & - \\
\hline HD 17548 & 6011 & 4.44 & 1.18 & -0.53 & 9.84 & 0.88 & 2.45 & 0.04 & - \\
\hline HD 176666 & 6103 & 4.63 & 1.18 & -0.37 & 5.76 & 0.95 & 2.62 & 0.06 & - \\
\hline HD 177122 & 6021 & 4.52 & 1.03 & -0.10 & 2.15 & 1.04 & 2.44 & 0.04 & - \\
\hline HD 179346 & 6229 & 4.76 & 1.35 & -0.03 & 0.99 & 1.12 & 2.70 & 0.05 & - \\
\hline HD 18083 & 6144 & 4.70 & 1.33 & 0.03 & 0.96 & 1.11 & 2.70 & 0.05 & - \\
\hline HD 181428 & 6151 & 4.45 & 1.29 & 0.06 & 3.12 & 1.18 & 2.78 & 0.04 & - \\
\hline HD 188815 & 6217 & 4.34 & 1.31 & -0.53 & 8.76 & 0.95 & 2.52 & 0.08 & - \\
\hline HD 191033 & 6206 & 4.47 & 1.35 & -0.19 & 4.49 & 1.07 & $<1.30$ & - & - \\
\hline HD 192865 & 6307 & 4.44 & 1.59 & 0.13 & 2.26 & 1.32 & $<0.80$ & - & 5.11 \\
\hline HD 195200 & 6201 & 4.44 & 1.25 & -0.08 & 3.67 & 1.13 & 2.80 & 0.05 & - \\
\hline HD 196384 & 6611 & 4.79 & 1.78 & -0.13 & 0.77 & 1.21 & 2.48 & 0.15 & - \\
\hline HD 197300 & 6022 & 4.69 & 1.21 & 0.02 & 1.11 & 1.06 & 2.84 & 0.07 & - \\
\hline HD 199086 & 6149 & 4.65 & 1.21 & 0.18 & 0.86 & 1.17 & 2.58 & 0.05 & - \\
\hline HD 199289 & 5925 & 4.62 & 1.30 & -0.98 & 10.83 & 0.79 & 2.05 & 0.08 & - \\
\hline HD 199868 & 6152 & 4.45 & 1.26 & -0.13 & 4.82 & 1.09 & 2.58 & 0.05 & - \\
\hline HD 200538 & 6042 & 4.38 & 1.22 & 0.10 & 3.58 & 1.14 & 2.01 & 0.06 & - \\
\hline HD 201496 & 5974 & 4.44 & 1.12 & -0.04 & 3.06 & 1.04 & 1.93 & 0.15 & - \\
\hline HD 202209 & 6009 & 4.68 & 1.19 & -0.01 & 1.38 & 1.05 & 2.78 & 0.04 & - \\
\hline HD 202871 & 6055 & 4.54 & 1.04 & -0.09 & 1.99 & 1.06 & 2.72 & 0.03 & - \\
\hline
\end{tabular}


Table 4. continued.

\begin{tabular}{|c|c|c|c|c|c|c|c|c|c|}
\hline Star & $\begin{array}{l}T_{\text {eff }} \\
(\mathrm{K})\end{array}$ & $\begin{array}{c}\log g \\
\left(\mathrm{~cm} \mathrm{~s}^{-2}\right)\end{array}$ & $\begin{array}{c}\xi_{t} \\
\left(\mathrm{~km} \mathrm{~s}^{-1}\right)\end{array}$ & {$[\mathrm{Fe} / \mathrm{H}]$} & $\begin{array}{r}\text { Age } \\
\text { (Gyr) }\end{array}$ & $\begin{array}{l}\text { Mass } \\
\left(M_{\odot}\right) \\
\end{array}$ & $A(\mathrm{Li})$ & Error & $\begin{array}{c}v \sin i \\
\left(\mathrm{~km} \mathrm{~s}^{-1}\right) \\
\end{array}$ \\
\hline HD 203335 & 6306 & 4.56 & 1.44 & -0.04 & 2.42 & 1.19 & 2.66 & 0.05 & - \\
\hline HD 205294 & 6370 & 4.30 & 1.71 & -0.25 & 4.01 & 1.20 & 2.31 & 0.05 & 4.82 \\
\hline HD 205591 & 6575 & 4.75 & 1.85 & -0.08 & 1.38 & 1.25 & 2.75 & 0.06 & 7.68 \\
\hline HD 206116 & 6231 & 4.57 & 1.36 & 0.24 & 1.08 & 1.24 & 2.69 & 0.06 & - \\
\hline HD 207190 & 6182 & 4.33 & 1.51 & -0.42 & 7.35 & 0.99 & 2.64 & 0.04 & - \\
\hline HD 208 & 5914 & 4.47 & 1.05 & -0.31 & 9.07 & 0.91 & 2.04 & 0.04 & 2.59 \\
\hline HD 208068 & 6007 & 4.64 & 1.17 & -0.38 & 3.18 & 0.93 & $<0.90$ & - & 5.02 \\
\hline HD 20852 & 6813 & 4.76 & 2.34 & -0.35 & 2.39 & 1.20 & $<1.00$ & - & 6.08 \\
\hline HD 208672 & 5986 & 4.61 & 1.07 & 0.13 & 1.30 & 1.10 & 2.62 & 0.10 & - \\
\hline HD 211317 & 5965 & 4.30 & 1.21 & 0.27 & 3.87 & 1.19 & 2.62 & 0.05 & - \\
\hline HD 21132 & 6243 & 4.60 & 1.44 & -0.37 & 5.47 & 0.99 & 2.63 & 0.03 & 3.11 \\
\hline HD 21161 & 5923 & 4.24 & 1.14 & 0.09 & 5.28 & 1.09 & 2.12 & 0.08 & - \\
\hline HD 214094 & 6288 & 4.28 & 1.46 & -0.01 & 2.77 & 1.33 & $<0.30$ & - & 4.69 \\
\hline HD 215625 & 6282 & 4.58 & 1.35 & 0.10 & 0.71 & 1.19 & 2.82 & 0.05 & - \\
\hline HD 215906 & 6259 & 4.56 & 1.55 & -0.28 & 5.23 & 1.05 & 2.55 & 0.08 & - \\
\hline HD 217395 & 5916 & 4.52 & 0.95 & -0.13 & 2.86 & 1.00 & 2.21 & 0.05 & - \\
\hline HD 217958 & 5970 & 4.45 & 1.29 & 0.28 & 1.93 & 1.13 & 2.30 & 0.15 & - \\
\hline HD 218379 & 5938 & 4.11 & 1.24 & 0.15 & 3.72 & 1.27 & 2.46 & 0.04 & - \\
\hline HD 21977 & 5930 & 4.45 & 1.03 & 0.10 & 2.37 & 1.08 & $<1.87$ & 0.10 & - \\
\hline HD 220894 & 6282 & 4.60 & 1.35 & 0.02 & 1.00 & 1.18 & 2.34 & 0.05 & - \\
\hline HD 221638 & 6360 & 4.53 & 1.43 & -0.21 & 2.23 & 1.10 & 2.58 & 0.08 & - \\
\hline HD 224578 & 6158 & 4.67 & 1.13 & -0.01 & 1.14 & 1.11 & 2.83 & 0.07 & - \\
\hline HD 225297 & 6181 & 4.55 & 1.24 & -0.09 & 2.46 & 1.11 & 2.65 & 0.05 & - \\
\hline HD 23030 & 5951 & 4.37 & 1.22 & 0.20 & 3.98 & 1.16 & 2.58 & 0.08 & - \\
\hline HD 24062 & 6107 & 4.62 & 1.34 & 0.28 & 2.63 & 1.23 & 2.85 & 0.05 & - \\
\hline HD 24085 & 6065 & 4.47 & 1.22 & 0.17 & 2.59 & 1.17 & 2.68 & 0.08 & - \\
\hline HD 24112 & 6175 & 4.35 & 1.26 & 0.16 & 2.60 & 1.26 & 2.58 & 0.05 & 3.10 \\
\hline HD 25587 & 6258 & 4.61 & 1.78 & -0.12 & 4.02 & 1.18 & 2.23 & 0.15 & - \\
\hline HD 2567 & 6038 & 4.44 & 1.19 & 0.22 & 2.36 & 1.17 & 2.52 & 0.05 & - \\
\hline HD 25912 & 5900 & 4.52 & 0.99 & 0.12 & 1.37 & 1.06 & 2.15 & 0.06 & - \\
\hline HD 26887 & 6016 & 4.46 & 1.00 & -0.35 & 4.05 & 0.94 & 2.30 & 0.07 & - \\
\hline HD 28969 & 6255 & 4.68 & 1.47 & -0.01 & 1.07 & 1.15 & 2.84 & 0.06 & - \\
\hline HD 29980 & 6019 & 4.71 & 1.57 & 0.12 & 1.78 & 1.12 & 2.71 & 0.08 & - \\
\hline HD 30053 & 6139 & 4.51 & 1.20 & -0.22 & 3.34 & 1.03 & 2.52 & 0.05 & - \\
\hline HD 31103 & 6078 & 4.49 & 1.08 & 0.09 & 1.09 & 1.12 & $<0.80$ & - & 3.18 \\
\hline HD 3229 & 6583 & 4.14 & 1.80 & -0.09 & 1.80 & 1.56 & $<2.00$ & - & - \\
\hline HD 32804 & 5910 & 4.53 & 1.08 & 0.06 & 1.71 & 1.05 & 2.72 & 0.03 & - \\
\hline HD 33081 & 6399 & 4.56 & 2.47 & -0.16 & 3.38 & 1.19 & $<1.50$ & - & - \\
\hline HD 36051 & 6118 & 4.63 & 1.21 & -0.08 & 1.26 & 1.07 & 2.77 & 0.05 & - \\
\hline HD 37226 & 6178 & 4.16 & 1.61 & -0.12 & 2.77 & 1.39 & $<0.70$ & - & 4.47 \\
\hline HD 37548 & 5950 & 4.26 & 1.19 & -0.04 & 6.10 & 1.09 & 2.20 & 0.06 & - \\
\hline HD 37990 & 6215 & 4.56 & 1.15 & 0.00 & 1.08 & 1.14 & 2.83 & 0.05 & - \\
\hline HD 38385 & 7212 & 4.61 & 2.87 & 0.09 & 0.61 & 1.56 & 2.40 & 0.15 & - \\
\hline HD 38772 & 6106 & 4.37 & 1.16 & -0.23 & 6.72 & 1.03 & 2.55 & 0.07 & - \\
\hline HD 40483 & 6371 & 4.39 & 1.80 & -0.06 & 2.68 & 1.34 & $<1.30$ & - & - \\
\hline HD 457 & 6089 & 4.43 & 1.17 & 0.34 & 2.45 & 1.21 & 2.59 & 0.04 & - \\
\hline HD 4597 & 6025 & 4.43 & 1.11 & -0.39 & 8.38 & 0.92 & 2.31 & 0.08 & - \\
\hline HD 52449 & 6362 & 4.55 & 1.29 & 0.12 & 0.49 & 1.21 & 2.90 & 0.08 & - \\
\hline HD 5388 & 6311 & 4.24 & 1.65 & -0.28 & 3.90 & 1.22 & 1.46 & 0.10 & 4.66 \\
\hline HD 54521 & 5973 & 4.54 & 1.02 & -0.01 & 2.01 & 1.05 & 2.30 & 0.10 & - \\
\hline HD 564 & 5902 & 4.53 & 0.95 & -0.20 & 5.21 & 0.96 & 2.30 & 0.04 & 2.44 \\
\hline HD 62364 & 6255 & 4.47 & 1.42 & -0.11 & 3.99 & 1.16 & 2.61 & 0.06 & - \\
\hline HD 63754 & 6200 & 4.21 & 1.57 & 0.21 & 2.36 & 1.41 & 2.30 & 0.05 & - \\
\hline HD 65982 & 5947 & 4.42 & 1.08 & -0.10 & 4.91 & 1.01 & 2.15 & 0.05 & - \\
\hline HD 66039 & 6149 & 4.52 & 1.14 & 0.17 & 0.92 & 1.18 & 2.85 & 0.04 & - \\
\hline HD 66168 & 6198 & 4.69 & 1.18 & -0.03 & 1.03 & 1.11 & 2.70 & 0.06 & - \\
\hline HD 66740 & 6666 & 4.49 & 1.70 & 0.04 & 0.87 & 1.35 & $<1.40$ & - & - \\
\hline HD 67200 & 6105 & 4.44 & 1.19 & 0.32 & 1.43 & 1.19 & 2.69 & 0.07 & - \\
\hline HD 68287 & 6318 & 4.63 & 1.45 & 0.06 & 0.95 & 1.21 & 2.91 & 0.05 & - \\
\hline HD 71685 & 6038 & 4.58 & 1.09 & -0.37 & 5.21 & 0.93 & 2.48 & 0.05 & - \\
\hline HD 74957 & 5915 & 4.54 & 0.95 & -0.18 & 6.54 & 0.97 & 1.93 & 0.08 & 3.07 \\
\hline HD 75328 & 6003 & 4.46 & 1.05 & -0.23 & 6.38 & 0.98 & 2.31 & 0.08 & - \\
\hline HD 75881 & 6239 & 4.44 & 1.63 & 0.07 & 2.69 & 1.33 & 2.73 & 0.07 & - \\
\hline HD 76188 & 5989 & 4.08 & 1.25 & -0.44 & 7.64 & 1.01 & 2.53 & 0.07 & - \\
\hline HD 82114 & 5912 & 4.20 & 1.28 & 0.01 & 5.35 & 1.15 & 2.41 & 0.05 & - \\
\hline
\end{tabular}


Table 4. continued.

\begin{tabular}{lcccrrrrrc}
\hline \hline Star & $\begin{array}{c}T_{\text {eff }} \\
(\mathrm{K})\end{array}$ & $\begin{array}{c}\log g \\
\left(\mathrm{~cm} \mathrm{~s}^{-2}\right)\end{array}$ & $\begin{array}{c}\xi_{t} \\
\left(\mathrm{~km} \mathrm{~s}^{-1}\right)\end{array}$ & $\begin{array}{r}\mathrm{Fe} / \mathrm{H}] \\
\begin{array}{r}\text { Age } \\
(\mathrm{Gyr})\end{array}\end{array}$ & $\begin{array}{r}\text { Mass } \\
\left(M_{\odot}\right)\end{array}$ & $\begin{aligned} A(\mathrm{Li}) \\
\text { Error }\end{aligned}$ & $\begin{array}{c}v \sin i \\
\left(\mathrm{~km} \mathrm{~s}^{-1}\right)\end{array}$ \\
\hline HD 84305 & 5963 & 4.51 & 0.95 & -0.23 & 5.39 & 0.97 & 2.31 & 0.07 & - \\
HD 84627 & 6113 & 4.50 & 1.10 & -0.28 & 1.71 & 1.00 & 2.54 & 0.06 & - \\
HD 85725 & 5986 & 3.95 & 1.54 & 0.15 & 2.42 & 1.50 & $<0.56$ & - & 5.85 \\
HD 86652 & 5934 & 4.47 & 1.01 & 0.13 & 1.62 & 1.09 & 2.00 & 0.10 & - \\
HD 87838 & 6118 & 4.47 & 1.33 & -0.40 & 6.72 & 0.94 & 2.49 & 0.05 & - \\
HD 8985 & 6473 & 4.96 & 2.15 & -0.01 & 0.52 & 1.20 & 2.97 & 0.08 & - \\
HD 90081 & 5912 & 4.34 & 1.08 & -0.20 & 8.12 & 0.95 & 2.01 & 0.10 & - \\
HD 90936 & 5928 & 4.48 & 1.04 & 0.03 & 2.08 & 1.05 & 2.16 & 0.08 & - \\
HD 91379 & 6164 & 4.41 & 0.98 & -0.29 & 2.46 & 1.01 & 2.60 & 0.10 & - \\
HD 92547 & 6020 & 4.45 & 1.14 & -0.37 & 8.41 & 0.92 & 2.24 & 0.08 & - \\
HD 93745 & 6065 & 4.34 & 1.33 & 0.12 & 3.57 & 1.22 & 2.40 & 0.08 & - \\
HD 93932 & 5950 & 4.30 & 1.16 & 0.05 & 5.46 & 1.09 & 2.17 & 0.07 & - \\
HD 94964 & 6139 & 4.55 & 1.26 & -0.07 & 2.32 & 1.10 & 2.71 & 0.05 & - \\
HD 95542 & 5984 & 4.52 & 1.01 & -0.04 & 1.75 & 1.04 & 2.59 & 0.03 & - \\
HD 95922 & 6293 & 4.63 & 1.23 & -0.06 & 2.08 & 1.16 & 2.92 & 0.05 & - \\
HD 9608 & 5954 & 4.43 & 0.98 & -0.26 & 8.87 & 0.95 & 2.19 & 0.05 & - \\
HD 96276 & 6080 & 4.49 & 1.12 & -0.02 & 2.37 & 1.09 & 2.50 & 0.05 & - \\
HD 96290 & 6219 & 4.56 & 1.21 & 0.03 & 1.01 & 1.15 & 2.71 & 0.06 & - \\
HD 97320 & 6162 & 4.57 & 1.50 & -1.05 & 10.46 & 0.81 & 2.52 & 0.05 & - \\
HD 98284 & 5913 & 4.52 & 1.18 & -0.84 & 9.48 & 0.81 & 2.12 & 0.08 & - \\
HD 131664 & 5901 & 4.50 & 1.04 & 0.31 & 2.32 & 1.10 & 2.10 & 0.04 & 3.01 \\
\hline
\end{tabular}

Table 5. Li abundances for planet hosts not belonging to the HARPS-GTO sample.

\begin{tabular}{|c|c|c|c|c|c|c|c|c|c|c|c|c|}
\hline Star & $\begin{array}{l}T_{\text {eff }} \\
(\mathrm{K})\end{array}$ & $\begin{array}{c}\log g \\
\left(\mathrm{~cm} \mathrm{~s}^{-2}\right)\end{array}$ & $\begin{array}{c}\xi_{t} \\
\left(\mathrm{~km} \mathrm{~s}^{-1}\right)\end{array}$ & {$[\mathrm{Fe} / \mathrm{H}]$} & $\begin{array}{l}\text { Age } \\
(\mathrm{Gyr})\end{array}$ & $\begin{array}{l}\text { Mass } \\
\left(M_{\odot}\right)\end{array}$ & $A(\mathrm{Li})$ & Error & $\begin{array}{c}v \sin i \\
\left(\mathrm{~km} \mathrm{~s}^{-1}\right)\end{array}$ & Hot jupiter & Flag & Ref. \\
\hline HD 2039 & 5976 & 4.45 & 1.26 & 0.32 & 2.03 & 1.14 & 2.38 & 0.06 & 3.46 & no & [1] & [c] \\
\hline HD 8574 & 6151 & 4.51 & 1.45 & 0.06 & 2.75 & 1.17 & 2.65 & 0.10 & - & no & [4] & [c] \\
\hline HD 9826 & 6212 & 4.26 & 1.69 & 0.13 & 2.65 & 1.28 & 2.87 & 0.08 & - & yes & [4] & [c] \\
\hline HD 11506 & 6204 & 4.44 & 1.32 & 0.36 & 0.83 & 1.23 & 2.96 & 0.04 & 5.01 & no & [1] & [b] \\
\hline HD 13931 & 5940 & 4.42 & 1.19 & 0.08 & 3.15 & 1.08 & 1.84 & 0.15 & - & no & [7] & [b] \\
\hline HD 16175 & 6030 & 4.23 & 1.39 & 0.32 & 3.22 & 1.29 & 2.78 & 0.07 & 4.58 & no & [9] & [b] \\
\hline HD 17156 & 6084 & 4.33 & 1.47 & 0.23 & 2.77 & 1.21 & 2.84 & 0.05 & - & no & [7] & [e] \\
\hline HD 20367 & 6138 & 4.53 & 1.22 & 0.17 & 1.18 & 1.15 & 3.00 & 0.10 & - & no & [10] & [c] \\
\hline HD 23596 & 6108 & 4.25 & 1.30 & 0.31 & 2.77 & 1.24 & 2.83 & 0.05 & - & no & [11] & [c] \\
\hline HD 30562 & 5970 & 4.20 & 1.20 & 0.32 & 3.73 & 1.22 & 2.78 & 0.05 & - & no & [3] & [d] \\
\hline HD 31253 & 6147 & 4.27 & 1.47 & 0.17 & 2.77 & 1.25 & 1.75 & 0.07 & 3.25 & no & [3] & [b] \\
\hline HD 33283 & 6058 & 4.16 & 1.41 & 0.34 & 2.92 & 1.34 & 2.51 & 0.05 & 4.08 & no & [1] & [b] \\
\hline HD 34445 & 5915 & 4.30 & 1.11 & 0.24 & 4.36 & 1.14 & 1.80 & 0.08 & - & no & [5] & [d] \\
\hline HD 38283 & 5980 & 4.27 & 1.28 & -0.14 & 7.43 & 1.06 & 2.43 & 0.05 & 3.80 & no & [3] & [b] \\
\hline HD 40979 & 6145 & 4.31 & 1.29 & 0.21 & 0.79 & 1.19 & 2.96 & 0.07 & - & no & [4] & [c] \\
\hline HD 43691 & 6200 & 4.28 & 1.49 & 0.28 & 2.28 & 1.28 & $<2.21$ & - & - & no & [7] & [i] \\
\hline HD 50499 & 6056 & 4.29 & 1.23 & 0.39 & 2.93 & 1.20 & 2.70 & 0.07 & - & no & [5] & [d] \\
\hline HD 50554 & 6026 & 4.41 & 1.11 & 0.01 & 1.96 & 1.09 & 2.52 & 0.05 & 3.93 & no & [11] & [c] \\
\hline HD 60532 & 6273 & 4.02 & 1.88 & -0.09 & 2.24 & 1.50 & 1.90 & 0.08 & 5.83 & no & [2] & [b] \\
\hline HD 68988 & 5988 & 4.45 & 1.25 & 0.36 & 1.38 & 1.14 & 2.25 & 0.10 & - & no & [4] & [c] \\
\hline HD 74156 & 6112 & 4.34 & 1.38 & 0.16 & 2.96 & 1.25 & 2.68 & 0.04 & 4.31 & no & [3] & [c] \\
\hline HD 75898 & 6137 & 4.31 & 1.36 & 0.30 & 2.54 & 1.25 & 2.85 & 0.06 & 4.32 & no & [1] & [b] \\
\hline HD 86081 & 6036 & 4.21 & 1.34 & 0.22 & 2.68 & 1.19 & 2.00 & 0.08 & 4.82 & yes & [1] & [b] \\
\hline HD 86226 & 5947 & 4.54 & 1.12 & 0.02 & 1.05 & 1.08 & 2.17 & 0.06 & - & no & [3] & [b] \\
\hline HD 86264 & 6596 & 4.47 & 1.90 & 0.37 & 0.81 & 1.40 & $<1.15$ & - & - & no & [3] & [b] \\
\hline HD 89307 & 5967 & 4.51 & 1.33 & -0.13 & 3.54 & 1.02 & 2.31 & 0.08 & - & no & [4] & [d] \\
\hline HD 95128 & 5954 & 4.44 & 1.30 & 0.06 & 4.44 & 1.07 & 1.85 & 0.15 & - & no & [4] & [c] \\
\hline HD 103774 & 6732 & 4.81 & 2.03 & 0.29 & 0.26 & 1.40 & $<1.30$ & - & 7.95 & no & [2] & [b] \\
\hline HD 118203 & 5910 & 4.18 & 1.34 & 0.25 & 3.61 & 1.29 & 2.70 & 0.05 & - & no & [4] & [b] \\
\hline HD 120136 & 6339 & 4.19 & 1.70 & 0.23 & 1.49 & 1.30 & $<1.40$ & - & 14.20 & no & [1] & [c] \\
\hline HD 142415 & 6045 & 4.53 & 1.12 & 0.21 & 0.51 & 1.12 & 2.10 & 0.10 & - & no & [3] & {$[\mathrm{c}]$} \\
\hline
\end{tabular}

Notes. The instrument used and the reference for the stellar parameters are shown in the last two columns. Flag: [1] UVES; [2] HARPS; [3] FEROS; [4] SARG; [5] CORALIE; [6] NOT; [7] SOPHIE; [8] HIRES; [9] FIES; [10] ELODIE; [11] UES.

References. [a] Mortier et al. (2013); [b] Santos et al. (2013); [c] Santos et al. (2004); [d] Sousa et al. (2006); [e] Ammler-von Eiff et al. (2009); [f] Santos et al. (2005); [g] Santos et al. (2006); [h]Moutou et al. (2006); [i] da Silva et al. (2007); [j] Molenda-Żakowicz et al. (2013); [k] Bonomo et al. (2012); [1] Pont et al. (2008); [m] Udalski et al. (2008). 
Table 5. continued.

\begin{tabular}{|c|c|c|c|c|c|c|c|c|c|c|c|c|}
\hline Star & $\begin{array}{l}T_{\text {eff }} \\
(\mathrm{K}) \\
\end{array}$ & $\begin{array}{c}\log g \\
\left(\mathrm{~cm} \mathrm{~s}^{-2}\right)\end{array}$ & $\begin{array}{c}\xi_{t} \\
\left(\mathrm{~km} \mathrm{~s}^{-1}\right)\end{array}$ & {$[\mathrm{Fe} / \mathrm{H}]$} & $\begin{array}{r}\text { Age } \\
\text { (Gyr) }\end{array}$ & $\begin{array}{l}\text { Mass } \\
\left(M_{\odot}\right) \\
\end{array}$ & $A(\mathrm{Li})$ & Error & $\begin{array}{c}v \sin i \\
\left(\mathrm{~km} \mathrm{~s}^{-1}\right)\end{array}$ & Hot jupiter & Flag & Ref. \\
\hline HD 149026 & 6162 & 4.37 & 1.41 & 0.36 & 2.34 & 1.25 & 2.44 & 0.10 & - & yes & [4] & {$[\mathrm{e}]$} \\
\hline HD 149143 & 6018 & 4.31 & 1.12 & 0.45 & 2.92 & 1.17 & 1.95 & 0.10 & - & yes & [5] & [d] \\
\hline HD 150706 & 5961 & 4.50 & 1.11 & -0.01 & 0.56 & 1.05 & 2.63 & 0.04 & - & no & [11] & [c] \\
\hline HD 155358 & 5908 & 4.26 & 1.29 & -0.62 & 11.74 & 0.88 & 2.14 & 0.10 & 3.12 & no & [1] & [b] \\
\hline HD 164509 & 5957 & 4.43 & 1.09 & 0.24 & 0.93 & 1.13 & 2.30 & 0.06 & 3.60 & no & [2] & [b] \\
\hline HD 176051 & 6030 & 4.68 & 1.28 & -0.04 & 3.39 & 1.07 & 2.54 & 0.06 & - & no & [7] & [b] \\
\hline HD 183263 & 5991 & 4.38 & 1.23 & 0.34 & 1.66 & 1.14 & 2.36 & 0.05 & - & no & [3] & [f] \\
\hline HD 185269 & 5983 & 4.05 & 1.49 & 0.10 & 3.75 & 1.27 & 2.49 & 0.05 & - & no & [10] & [h] \\
\hline HD 187085 & 6146 & 4.36 & 1.31 & 0.13 & 1.76 & 1.18 & 2.66 & 0.06 & 3.74 & no & {$[1]$} & [b] \\
\hline HD 196067 & 5999 & 4.13 & 1.30 & 0.23 & 3.39 & 1.23 & 2.40 & 0.06 & - & no & [3] & [b] \\
\hline HD 196885A & 6340 & 4.46 & 1.51 & 0.29 & 0.52 & 1.27 & 2.74 & 0.05 & - & no & [4] & [d] \\
\hline HD 205739 & 6301 & 4.40 & 1.42 & 0.21 & 1.74 & 1.31 & 2.71 & 0.04 & 4.06 & no & [1] & [b] \\
\hline HD 220689 & 5904 & 4.38 & 1.13 & -0.01 & 4.28 & 1.03 & 1.92 & 0.06 & - & no & [3] & [b] \\
\hline HD 220773 & 5995 & 4.26 & 1.33 & 0.11 & 4.15 & 1.21 & 2.48 & 0.05 & - & no & [3] & [b] \\
\hline HD 224693 & 6053 & 4.18 & 1.40 & 0.28 & 3.00 & 1.32 & 2.09 & 0.08 & 4.20 & no & [1] & [b] \\
\hline HD 231701 & 6224 & 4.37 & 1.35 & 0.04 & 1.35 & 1.18 & 2.77 & 0.04 & 4.26 & no & [1] & [b] \\
\hline CoRoT-1 & 6397 & 4.66 & 1.68 & 0.03 & 0.95 & 1.39 & 2.77 & 0.06 & 4.74 & no & [2] & [a] \\
\hline CoRoT-4 & 6344 & 4.82 & 1.74 & 0.15 & 1.20 & 1.28 & $<2.43$ & - & 6.40 & no & [2] & [a] \\
\hline CoRoT-5 & 6240 & 4.46 & 1.28 & 0.04 & 1.83 & 1.22 & 2.88 & 0.07 & 3.82 & yes & [2] & [a] \\
\hline HAT-P-1 & 6076 & 4.47 & 1.17 & 0.21 & 1.28 & 1.18 & 1.77 & 0.10 & - & yes & [4] & [e] \\
\hline HAT-P-4 & 6054 & 4.17 & 1.59 & 0.35 & 2.12 & 1.21 & 2.90 & 0.10 & - & yes & [7] & [e] \\
\hline HAT-P-6 & 6855 & 4.69 & 2.85 & -0.08 & 0.73 & 1.24 & $<1.90$ & - & - & no & [7] & [e] \\
\hline HAT-P-7 & 6525 & 4.09 & 1.78 & 0.31 & 1.05 & 1.42 & 3.29 & 0.10 & - & no & [7] & {$[\mathrm{e}]$} \\
\hline HAT-P-8 & 6550 & 4.80 & 1.93 & 0.07 & 0.53 & 1.23 & 3.02 & 0.10 & 10.94 & no & [9] & [a] \\
\hline HAT-P-30 & 6338 & 4.52 & 1.40 & 0.12 & 0.85 & 1.25 & 3.10 & 0.06 & - & no & [3] & [a] \\
\hline HAT-P-35 & 6178 & 4.40 & 1.34 & 0.12 & 1.61 & 1.21 & 2.67 & 0.05 & - & yes & [3] & [a] \\
\hline Kepler-21 & 6409 & 4.43 & 1.86 & -0.03 & 2.42 & 1.36 & $<1.20$ & - & - & no & [7] & [j] \\
\hline Kepler-43 & 6041 & 4.26 & 1.85 & 0.33 & 2.34 & 1.19 & 2.20 & 0.15 & - & yes & [7] & [k] \\
\hline OGLE-TR-10 & 6075 & 4.54 & 1.45 & 0.28 & 2.02 & 1.22 & 2.28 & 0.10 & 6.43 & yes & [1] & [g] \\
\hline OGLE-TR-182 & 5924 & 4.47 & 0.91 & 0.37 & 2.78 & 1.16 & $<1.89$ & - & 4.53 & no & [1] & [1] \\
\hline OGLE-TR-211 & 6325 & 4.22 & 1.63 & 0.11 & - & - & $<1.50$ & - & 7.10 & no & [1] & [m] \\
\hline OGLE-TR-56 & 6119 & 4.21 & 1.48 & 0.25 & 1.80 & 1.25 & 2.63 & 0.10 & 5.25 & yes & [1] & {$[\mathrm{g}]$} \\
\hline TrES-4 & 6293 & 4.20 & 2.01 & 0.34 & 1.36 & 1.31 & $<1.50$ & - & - & no & [7] & [e] \\
\hline WASP-1 & 6252 & 4.32 & 1.42 & 0.23 & 1.17 & 1.29 & 3.29 & 0.08 & 3.66 & yes & [1] & [a] \\
\hline WASP-7 & 6621 & 4.62 & 3.00 & 0.12 & 0.71 & 1.22 & $<1.70$ & - & 17.48 & no & [1] & [a] \\
\hline WASP-12 & 6313 & 4.37 & 1.65 & 0.21 & 1.18 & 1.30 & 2.65 & 0.08 & - & no & [3] & [a] \\
\hline WASP-13 & 6025 & 4.19 & 1.28 & 0.11 & 2.93 & 1.16 & 2.17 & 0.08 & 4.35 & yes & [8] & [a] \\
\hline WASP-15 & 6573 & 4.79 & 1.72 & 0.09 & 0.52 & 1.30 & $<2.00$ & - & 4.77 & no & [2] & [a] \\
\hline WASP-17 & 6794 & 4.83 & 2.57 & -0.12 & 0.74 & 1.27 & $<1.30$ & - & 8.87 & no & [2] & [a] \\
\hline WASP-18 & 6526 & 4.73 & 1.83 & 0.19 & 0.33 & 1.28 & 2.81 & 0.10 & - & no & [3] & [a] \\
\hline WASP-21 & 5924 & 4.39 & 1.06 & -0.22 & 5.29 & 0.96 & 2.28 & 0.10 & - & yes & [3] & [a] \\
\hline WASP-22 & 6153 & 4.57 & 1.36 & 0.26 & 1.31 & 1.22 & 2.49 & 0.10 & 4.29 & yes & [2] & [a] \\
\hline WASP-24 & 6297 & 4.76 & 1.41 & 0.09 & 0.84 & 1.21 & 2.52 & 0.08 & 5.55 & yes & [2] & [a] \\
\hline WASP-26 & 6034 & 4.44 & 1.28 & 0.16 & 1.85 & 1.16 & 2.09 & 0.10 & - & yes & [3] & [a] \\
\hline WASP-28 & 6134 & 4.55 & 1.17 & -0.12 & 2.68 & 1.09 & 2.51 & 0.10 & 4.39 & yes & [2] & [a] \\
\hline WASP-31 & 6443 & 4.76 & 1.62 & -0.08 & 1.16 & 1.18 & 2.97 & 0.08 & 6.97 & no & [2] & [a] \\
\hline WASP-32 & 6427 & 4.93 & 1.20 & 0.28 & 0.73 & 1.20 & $<1.80$ & - & - & no & [3] & [a] \\
\hline WASP-35 & 6072 & 4.69 & 1.26 & -0.05 & 1.82 & 1.07 & 2.38 & 0.08 & - & yes & [3] & [a] \\
\hline WASP-36 & 5928 & 4.51 & 0.89 & -0.01 & 3.56 & 1.05 & $<1.94$ & - & 4.01 & no & [2] & [a] \\
\hline WASP-38 & 6436 & 4.80 & 1.75 & 0.06 & 0.35 & 1.21 & $<2.20$ & - & 7.43 & no & [2] & [a] \\
\hline WASP-54 & 6296 & 4.37 & 1.45 & 0.00 & 1.51 & 1.20 & $<1.30$ & - & - & no & [3] & [a] \\
\hline WASP-55 & 6070 & 4.55 & 1.10 & 0.09 & 1.90 & 1.14 & 2.51 & 0.08 & - & yes & [3] & [a] \\
\hline WASP-62 & 6391 & 4.73 & 1.50 & 0.24 & 0.50 & 1.26 & 2.63 & 0.06 & - & no & [3] & [a] \\
\hline WASP-66 & 7051 & 5.00 & 3.07 & 0.05 & 0.38 & 1.45 & 3.39 & 0.06 & - & no & [3] & [a] \\
\hline WASP-71 & 6180 & 4.15 & 1.69 & 0.37 & 1.68 & 1.27 & 2.33 & 0.10 & - & yes & [3] & [a] \\
\hline WASP-78 & 6291 & 4.19 & 1.63 & -0.07 & 2.21 & 1.19 & $<1.50$ & - & - & no & [3] & [a] \\
\hline WASP-79 & 7002 & 4.77 & 2.64 & 0.19 & 0.39 & 1.36 & $<2.30$ & - & - & no & [3] & [a] \\
\hline
\end{tabular}


E. Delgado Mena et al.: Li abundances in F stars: planets, rotation, and Galactic evolution

Table 6. Li abundances for cool stars without detected planets from HARPS GTO samples.

\begin{tabular}{|c|c|c|c|c|c|c|c|}
\hline Star & $\begin{array}{l}T_{\text {eff }} \\
(\mathrm{K}) \\
\end{array}$ & $\begin{array}{c}\log g \\
\left(\mathrm{~cm} \mathrm{~s}^{-2}\right)\end{array}$ & $\begin{array}{c}\xi_{t} \\
\left(\mathrm{~km} \mathrm{~s}^{-1}\right)\end{array}$ & {$[\mathrm{Fe} / \mathrm{H}]$} & $\begin{array}{r}\text { Age } \\
\text { (Gyr) }\end{array}$ & $\begin{array}{l}\text { Mass } \\
\left(M_{\odot}\right) \\
\end{array}$ & $A(\mathrm{Li})$ \\
\hline HD 100289 & 5483 & 4.42 & 0.70 & 0.03 & 4.09 & 0.92 & $<0.35$ \\
\hline HD 102136 & 5349 & 4.43 & 0.75 & -0.09 & 3.92 & 0.86 & $<0.00$ \\
\hline HD 102843 & 5432 & 4.35 & 0.79 & 0.16 & 3.04 & 0.92 & $<-0.21$ \\
\hline HD 103720 & 5017 & 4.43 & 0.90 & -0.02 & 4.00 & 0.79 & $<-0.51$ \\
\hline HD 106589 & 5597 & 4.37 & 0.67 & -0.23 & 6.83 & 0.88 & $<0.53$ \\
\hline HD 107094 & 5564 & 4.54 & 0.75 & -0.51 & 5.28 & 0.81 & 1.95 \\
\hline HD 108341 & 5122 & 4.45 & 0.64 & 0.04 & 4.70 & 0.84 & $<-0.21$ \\
\hline HD 109988 & 5193 & 4.46 & 0.81 & 0.14 & 5.06 & 0.88 & $<0.15$ \\
\hline HD 110291 & 5480 & 4.38 & 0.69 & -0.02 & 4.17 & 0.90 & $<0.31$ \\
\hline HD 110557 & 5267 & 4.36 & 0.71 & -0.06 & 4.07 & 0.84 & $<-0.69$ \\
\hline HD 112100 & 5081 & 4.44 & 0.50 & -0.16 & 4.39 & 0.79 & $<-0.51$ \\
\hline HD 113569 & 4994 & 4.41 & 0.41 & -0.22 & 4.58 & 0.76 & $<-0.20$ \\
\hline HD 114076 & 5069 & 4.32 & 0.04 & -0.47 & 5.56 & 0.74 & $<-0.37$ \\
\hline HD 115499 & 5542 & 4.45 & 0.95 & 0.07 & 3.51 & 0.94 & $<1.57$ \\
\hline HD 11608 & 4959 & 4.32 & 0.56 & 0.22 & 4.28 & 0.82 & $<0.06$ \\
\hline HD 116883 & 4902 & 4.36 & 0.52 & -0.20 & 4.75 & 0.75 & $<-0.28$ \\
\hline HD 1171 & 5293 & 4.47 & 0.48 & -0.53 & 4.18 & 0.75 & $<0.00$ \\
\hline HD 117359 & 5246 & 4.43 & 0.68 & -0.13 & 4.90 & 0.83 & $<-1.02$ \\
\hline HD 118466 & 5049 & 4.34 & 0.49 & 0.20 & 4.93 & 0.86 & $<0.06$ \\
\hline HD 118563 & 5477 & 4.45 & 0.68 & -0.04 & 3.59 & 0.89 & $<0.23$ \\
\hline HD 119503 & 4885 & 4.40 & 0.83 & -0.04 & 4.37 & 0.77 & $<-0.07$ \\
\hline HD 120362 & 5517 & 4.51 & 1.05 & 0.10 & 2.68 & 0.93 & $<0.78$ \\
\hline HD 122308 & 5253 & 4.44 & 0.59 & -0.32 & 4.35 & 0.78 & $<0.04$ \\
\hline HD 125522 & 4839 & 4.45 & 0.40 & -0.46 & 4.39 & 0.70 & $<-0.30$ \\
\hline HD 127124 & 5079 & 4.43 & 0.82 & -0.04 & 4.84 & 0.82 & $<-0.37$ \\
\hline HD 128113 & 4922 & 4.28 & 0.35 & -0.17 & 5.01 & 0.77 & $<-0.32$ \\
\hline HD 128431 & 5429 & 4.43 & 0.61 & -0.34 & 5.01 & 0.82 & $<0.14$ \\
\hline HD 13252 & 5358 & 4.33 & 0.59 & -0.25 & 4.63 & 0.83 & $<0.11$ \\
\hline HD 132569 & 5026 & 4.49 & 0.59 & -0.26 & 4.29 & 0.75 & $<-0.37$ \\
\hline HD 133633 & 5571 & 4.48 & 0.69 & -0.45 & 7.13 & 0.82 & $<0.24$ \\
\hline HD 134929 & 5330 & 4.36 & 0.79 & 0.07 & 4.70 & 0.90 & $<-0.22$ \\
\hline HD 138799 & 5224 & 36 & 0.71 & 0.02 & 5.26 & 0.87 & $<0.38$ \\
\hline HD 138914 & 4983 & 4.38 & 0.66 & -0.12 & 4.81 & 0.78 & $<-0.32$ \\
\hline HD 139710 & 5123 & 4.41 & 0.90 & -0.08 & 4.09 & 0.81 & $<0.02$ \\
\hline HD 141598 & 5593 & 4.37 & 0.75 & -0.10 & 6.19 & 0.91 & $<0.20$ \\
\hline HD 144342 & 5403 & 4.47 & 0.90 & 0.07 & 4.09 & 0.90 & $<-0.07$ \\
\hline HD 147195 & 5557 & 4.48 & 0.68 & -0.05 & 6.05 & 0.92 & $<0.49$ \\
\hline HD 150474 & 5425 & 4.01 & 0.96 & 0.01 & 7.60 & 1.09 & 1.75 \\
\hline HD 155717 & 4949 & 4.48 & 0.76 & -0.13 & 3.96 & 0.76 & $<-0.37$ \\
\hline HD 15612 & 5256 & 4.49 & 0.87 & -0.11 & 4.17 & 0.83 & $<-1.01$ \\
\hline HD 156423 & 5184 & 4.46 & 0.21 & -0.43 & 4.76 & 0.75 & $<0.15$ \\
\hline HD 156517 & 5013 & 4.45 & 0.81 & 0.03 & 4.41 & 0.81 & $<0.03$ \\
\hline HD 157668 & 5195 & 4.49 & 0.65 & -0.23 & 4.48 & 0.79 & $<-0.03$ \\
\hline HD 15906 & 4884 & 4.49 & 0.65 & -0.01 & 4.33 & 0.78 & $<-0.41$ \\
\hline HD 16536 & 5282 & 4.39 & 0.70 & -0.08 & 4.57 & 0.85 & $<0.23$ \\
\hline HD 168769 & 5361 & 4.45 & 0.87 & -0.01 & 3.62 & 0.87 & $<0.26$ \\
\hline HD 168870 & 5325 & 4.43 & 0.67 & -0.32 & 4.89 & 0.81 & $<-0.15$ \\
\hline HD 170958 & 5599 & 4.83 & 1.38 & -0.04 & 3.05 & 0.91 & 2.39 \\
\hline HD 171825 & 4908 & 4.48 & 0.39 & -0.12 & 4.25 & 0.76 & $<-0.34$ \\
\hline HD 177033 & 4918 & 4.50 & 0.45 & -0.13 & 3.95 & 0.75 & $<-0.15$ \\
\hline HD 181249 & 4906 & 4.25 & 0.24 & -0.13 & 4.90 & 0.77 & $<-0.42$ \\
\hline HD 18777 & 5058 & 4.39 & 0.57 & 0.01 & 4.43 & 0.82 & $<0.00$ \\
\hline HD 188091 & 5120 & 4.32 & 0.87 & 0.08 & 4.53 & 0.85 & $<0.29$ \\
\hline HD 18822 & 5272 & 4.43 & 0.87 & -0.07 & 4.79 & 0.85 & $<1.13$ \\
\hline HD 18838 & 5500 & 4.48 & 0.64 & -0.17 & 4.16 & 0.87 & $<0.10$ \\
\hline HD 189004 & 5094 & 4.36 & 0.67 & -0.07 & 5.27 & 0.82 & $<-0.11$ \\
\hline HD 190204 & 5476 & 4.63 & 1.14 & -0.02 & 4.30 & 0.90 & $<1.55$ \\
\hline HD 191797 & 5061 & 4.50 & 0.86 & -0.06 & 4.82 & 0.81 & 1.31 \\
\hline HD 19230 & 5254 & 4.63 & 0.46 & -0.57 & 4.59 & 0.74 & $<-0.39$ \\
\hline HD 194717 & 5247 & 4.26 & 0.33 & -0.28 & 5.71 & 0.81 & $<0.25$ \\
\hline HD 196397 & 5378 & 4.33 & 0.68 & 0.29 & 4.08 & 0.93 & $<0.24$ \\
\hline HD 200143 & 5112 & 4.46 & 0.96 & 0.02 & 4.44 & 0.83 & $<-0.13$ \\
\hline HD 201161 & 4884 & 4.36 & 0.63 & -0.04 & 4.11 & 0.76 & $<-0.22$ \\
\hline
\end{tabular}

Notes. Parameters from Sousa et al. (2008, 2011a,b). 
Table 6. continued.

\begin{tabular}{|c|c|c|c|c|c|c|c|}
\hline Star & $\begin{array}{l}T_{\text {eff }} \\
(\mathrm{K})\end{array}$ & $\begin{array}{c}\log g \\
\left(\mathrm{~cm} \mathrm{~s}^{-2}\right)\end{array}$ & $\begin{array}{c}\xi_{t} \\
\left(\mathrm{~km} \mathrm{~s}^{-1}\right)\end{array}$ & {$[\mathrm{Fe} / \mathrm{H}]$} & $\begin{array}{c}\text { Age } \\
(\mathrm{Gyr})\end{array}$ & $\begin{array}{l}\text { Mass } \\
\left(M_{\odot}\right) \\
\end{array}$ & $A(\mathrm{Li})$ \\
\hline HD 2014 & 5054 & 4.37 & 0.64 & -0.07 & 4.43 & 0.80 & $<-0.16$ \\
\hline HD 203771 & 4963 & 4.43 & 0.50 & 0.13 & 4.23 & 0.81 & $<-0.27$ \\
\hline HD 203897 & 5184 & 4.42 & 0.65 & -0.18 & 4.14 & 0.80 & $<-0.61$ \\
\hline HD 206630 & 4853 & 4.48 & 0.06 & -0.41 & 4.44 & 0.70 & $<-0.49$ \\
\hline HD 209566 & 5500 & 4.38 & 0.75 & 0.12 & 4.86 & 0.94 & $<0.37$ \\
\hline HD 210329 & 4965 & 4.40 & 0.45 & -0.18 & 5.05 & 0.77 & $<-0.35$ \\
\hline HD 210507 & 4998 & 4.42 & 0.69 & 0.07 & 4.42 & 0.82 & $<0.04$ \\
\hline HD 211188 & 5053 & 4.41 & 0.42 & -0.12 & 4.29 & 0.79 & $<0.11$ \\
\hline HD 211534 & 5032 & 4.43 & 0.14 & -0.31 & 4.34 & 0.74 & $<-0.11$ \\
\hline HD 21251 & 4920 & 4.41 & 0.68 & -0.09 & 4.99 & 0.78 & $<-0.06$ \\
\hline HD 212918 & 5051 & 4.48 & 0.41 & -0.21 & 4.30 & 0.77 & $<-0.20$ \\
\hline HD 213852 & 4943 & 4.24 & 0.40 & 0.15 & 4.59 & 0.83 & $<0.27$ \\
\hline HD 215902 & 5454 & 4.46 & 0.53 & -0.25 & 4.24 & 0.84 & $<0.29$ \\
\hline HD 216215 & 5220 & 4.45 & 0.58 & -0.20 & 4.07 & 0.80 & $<0.34$ \\
\hline HD 217221 & 5184 & 4.45 & 0.77 & 0.01 & 4.46 & 0.85 & $<-0.09$ \\
\hline HD 21759 & 5142 & 4.49 & 0.43 & -0.61 & 4.52 & 0.72 & $<0.09$ \\
\hline HD 221974 & 5170 & 4.30 & 0.74 & 0.30 & 4.51 & 0.88 & $<0.30$ \\
\hline HD 222721 & 5361 & 4.43 & 0.52 & -0.31 & 5.13 & 0.82 & $<0.16$ \\
\hline HD 22282 & 5433 & 4.32 & 0.77 & 0.12 & 7.18 & 0.93 & $<0.59$ \\
\hline HD 224047 & 5167 & 4.43 & 0.62 & -0.23 & 4.89 & 0.79 & $<-0.02$ \\
\hline HD 224230 & 4944 & 4.47 & 0.56 & -0.10 & 4.07 & 0.76 & $<-0.29$ \\
\hline HD 224287 & 5330 & 4.38 & 0.59 & -0.29 & 4.37 & 0.81 & $<-0.02$ \\
\hline HD 224433 & 5527 & 4.42 & 0.72 & 0.09 & 3.37 & 0.94 & $<0.19$ \\
\hline HD 224685 & 5504 & 4.47 & 0.76 & -0.40 & 4.64 & 0.81 & $<0.50$ \\
\hline HD 24558 & 5274 & 4.40 & 0.67 & -0.47 & 5.48 & 0.77 & $<0.17$ \\
\hline HD 25061 & 5243 & 4.42 & 0.80 & 0.07 & 5.76 & 0.88 & $<0.21$ \\
\hline HD 25357 & 5117 & 4.72 & 1.02 & -0.03 & 3.48 & 0.80 & $<0.04$ \\
\hline HD 26430 & 4948 & 4.37 & 0.55 & -0.26 & 4.91 & 0.75 & $<-0.46$ \\
\hline HD 2768 & 5548 & 4.40 & 0.81 & -0.03 & 3.79 & 0.92 & $<0.76$ \\
\hline HD 290038 & 5006 & 4.42 & 0.56 & 0.01 & 4.64 & 0.81 & $<0.00$ \\
\hline HD 30669 & 5400 & 4.37 & 0.55 & 0.13 & 4.69 & 0.92 & $<0.46$ \\
\hline HD 30858 & 5182 & 4.45 & 0.88 & -0.13 & 4.59 & 0.81 & $<0.19$ \\
\hline HD 309701 & 4814 & 4.39 & 0.00 & -0.30 & 4.47 & 0.71 & $<-0.69$ \\
\hline HD 323631 & 4984 & 4.44 & 0.38 & -0.28 & 4.45 & 0.74 & $<-0.21$ \\
\hline HD 33811 & 5554 & 4.39 & 0.78 & 0.30 & 4.39 & 0.98 & $<0.26$ \\
\hline HD 36179 & 5327 & 4.45 & 0.69 & -0.08 & 3.77 & 0.85 & $<0.40$ \\
\hline HD 3808 & 5572 & 4.46 & 0.82 & -0.17 & 5.63 & 0.89 & $<0.96$ \\
\hline HD 38265 & 5549 & 4.43 & 0.72 & -0.14 & 5.69 & 0.90 & $<0.59$ \\
\hline HD 38355 & 5314 & 4.35 & 0.75 & 0.09 & 4.91 & 0.89 & $<0.43$ \\
\hline HD 40503 & 4953 & 4.29 & 0.81 & -0.03 & 4.93 & 0.80 & $<0.02$ \\
\hline HD 41087 & 5562 & 4.52 & 0.85 & -0.13 & 4.37 & 0.90 & 1.95 \\
\hline HD 4457 & 5015 & 4.53 & 0.65 & -0.37 & 4.82 & 0.74 & $<-0.08$ \\
\hline HD 44804 & 5366 & 4.48 & 0.97 & 0.03 & 4.40 & 0.89 & $<0.11$ \\
\hline HD 58676 & 5104 & 4.37 & 0.75 & -0.02 & 4.67 & 0.83 & $<0.36$ \\
\hline HD 62847 & 5362 & 4.48 & 0.67 & -0.25 & 3.94 & 0.82 & $<0.16$ \\
\hline HD 64640 & 5174 & 4.31 & 0.77 & 0.18 & 4.72 & 0.88 & $<0.18$ \\
\hline HD 66040 & 5226 & 4.34 & 0.76 & 0.35 & 4.43 & 0.89 & $<0.38$ \\
\hline HD 66340 & 5284 & 4.36 & 0.75 & 0.03 & 4.02 & 0.87 & $<-0.02$ \\
\hline HD 66838 & 5392 & 4.29 & 0.71 & 0.03 & 4.32 & 0.89 & $<0.45$ \\
\hline HD 70903 & 5118 & 4.52 & 0.63 & -0.43 & 5.29 & 0.75 & $<0.02$ \\
\hline HD 78964 & 5195 & 4.50 & 0.91 & 0.10 & 4.65 & 0.87 & 1.37 \\
\hline HD 81767 & 4978 & 4.46 & 0.68 & 0.05 & 4.62 & 0.82 & $<-0.13$ \\
\hline HD 88885 & 5361 & 4.46 & 0.77 & -0.11 & 4.59 & 0.86 & $<0.69$ \\
\hline HD 89147 & 5310 & 4.45 & 0.76 & -0.09 & 3.91 & 0.85 & $<0.42$ \\
\hline HD 89749 & 5443 & 4.48 & 0.61 & -0.29 & 5.17 & 0.83 & $<0.24$ \\
\hline HD 89920 & 4827 & 4.32 & 0.10 & -0.03 & 5.02 & 0.78 & $<-0.27$ \\
\hline HD 89965 & 5039 & 4.48 & 0.72 & -0.09 & 4.45 & 0.79 & $<0.02$ \\
\hline HD 90133 & 5064 & 4.42 & 0.37 & -0.16 & 5.07 & 0.79 & $<0.27$ \\
\hline HD 90926 & 5538 & 4.35 & 0.80 & 0.13 & 5.20 & 0.95 & $<0.25$ \\
\hline HD 91267 & 4928 & 4.48 & 0.26 & -0.06 & 4.73 & 0.79 & $<-0.19$ \\
\hline HD 93351 & 5408 & 4.41 & 0.57 & -0.23 & 5.24 & 0.85 & $<0.21$ \\
\hline HD 95533 & 5366 & 4.36 & 0.63 & 0.16 & 4.37 & 0.92 & $<0.20$ \\
\hline BD -050578 & 5470 & 4.39 & 0.79 & 0.09 & 2.88 & 0.91 & $<0.40$ \\
\hline BD -054065 & 4895 & 4.41 & 0.42 & -0.29 & 4.36 & 0.72 & $<-0.11$ \\
\hline BD -060904 & 5066 & 4.46 & 0.46 & -0.17 & 4.05 & 0.77 & $<0.10$ \\
\hline
\end{tabular}


Table 6. continued.

\begin{tabular}{|c|c|c|c|c|c|c|c|}
\hline Star & $\begin{array}{l}T_{\text {eff }} \\
(\mathrm{K}) \\
\end{array}$ & $\begin{array}{c}\log g \\
\left(\mathrm{~cm} \mathrm{~s}^{-2}\right)\end{array}$ & $\begin{array}{c}\begin{array}{c}\xi_{t} \\
\left(\mathrm{~km} \mathrm{~s}^{-1}\right)\end{array} \\
\end{array}$ & {$[\mathrm{Fe} / \mathrm{H}]$} & $\begin{array}{r}\text { Age } \\
(\mathrm{Gyr}) \\
\end{array}$ & $\begin{array}{l}\text { Mass } \\
\left(M_{\odot}\right) \\
\end{array}$ & $A(\mathrm{Li})$ \\
\hline BD -112763 & 4911 & 4.44 & 0.38 & -0.26 & 3.92 & 0.72 & $<-0.30$ \\
\hline BD -131161 & 5389 & 4.37 & 0.68 & 0.09 & 4.00 & 0.90 & $<0.35$ \\
\hline BD -160308 & 5183 & 4.48 & 0.51 & -0.41 & 4.55 & 0.75 & $<0.18$ \\
\hline BD -160931 & 4840 & 4.38 & 0.46 & -0.11 & 4.58 & 0.75 & $<-0.25$ \\
\hline HIP 10741 & 4859 & 4.40 & 0.51 & -0.15 & 3.89 & 0.73 & $<-0.12$ \\
\hline HIP 116939 & 4984 & 4.35 & 0.56 & -0.07 & 4.82 & 0.79 & $<0.43$ \\
\hline HIP 12147 & 5050 & 4.50 & 0.43 & -0.32 & 4.84 & 0.75 & $<-0.20$ \\
\hline HIP 15587 & 5239 & 4.46 & 0.66 & -0.12 & 3.63 & 0.81 & $<0.06$ \\
\hline HIP 16094 & 4877 & 4.44 & 0.39 & -0.04 & 4.04 & 0.76 & $<0.06$ \\
\hline HIP 1745 & 5416 & 4.39 & 0.47 & -0.44 & 4.47 & 0.79 & $<1.19$ \\
\hline HIP 20444 & 4907 & 4.41 & 0.54 & -0.12 & 3.96 & 0.75 & $<0.03$ \\
\hline HIP 32812 & 4977 & 4.41 & 0.56 & -0.02 & 4.25 & 0.79 & $<-0.01$ \\
\hline HIP 33392 & 4986 & 4.54 & 0.97 & -0.09 & 4.01 & 0.77 & $<0.13$ \\
\hline HIP 35992 & 4939 & 4.25 & 0.62 & -0.02 & 4.55 & 0.79 & $<-0.01$ \\
\hline HIP 5114 & 4846 & 4.39 & 0.74 & -0.69 & 4.36 & 0.70 & $<-0.49$ \\
\hline HIP 54446 & 4992 & 4.51 & 0.74 & -0.18 & 4.36 & 0.76 & $<1.02$ \\
\hline HIP 69224 & 5005 & 4.30 & 0.32 & -0.14 & 4.55 & 0.78 & $<0.32$ \\
\hline HIP 78242 & 5244 & 4.40 & 0.73 & -0.01 & 4.50 & 0.86 & $<0.31$ \\
\hline HD 109368 & 4651 & 4.36 & 0.67 & -0.23 & 4.33 & 0.70 & $<-0.30$ \\
\hline HD 116963 & 4735 & 4.43 & 0.51 & -0.06 & 4.16 & 0.73 & $<-0.10$ \\
\hline HD 117938 & 4738 & 4.27 & 0.36 & -0.13 & 4.78 & 0.74 & $<-0.10$ \\
\hline HD 119291 & 4611 & 4.22 & 0.59 & -0.10 & 4.15 & 0.70 & $<-0.27$ \\
\hline HD 11938 & 4703 & 4.25 & 0.76 & 0.01 & 4.41 & 0.75 & $<-0.07$ \\
\hline HD 120491 & 4680 & 4.49 & 0.40 & -0.34 & 4.22 & 0.70 & $<-0.11$ \\
\hline HD 125271 & 4779 & 4.33 & 0.36 & -0.22 & 4.36 & 0.71 & $<0.21$ \\
\hline HD 126829 & 4726 & 4.51 & 0.82 & -0.14 & 4.31 & 0.72 & $<-0.18$ \\
\hline HD 132411 & 4673 & 4.32 & 0.31 & -0.29 & 4.57 & 0.70 & $<-0.01$ \\
\hline HD 137010 & 4797 & 4.41 & 0.48 & -0.22 & 4.10 & 0.71 & $<0.00$ \\
\hline HD 151692 & 4737 & 4.40 & 0.55 & -0.07 & 4.23 & 0.73 & $<-0.08$ \\
\hline HD 152533 & 4822 & 4.36 & 0.40 & -0.04 & 4.38 & 0.76 & $<-0.21$ \\
\hline HD 154387 & 4719 & 4.50 & 0.08 & -0.25 & 4.27 & 0.70 & $<-0.03$ \\
\hline HD 160836 & 4791 & 4.49 & 0.92 & -0.16 & 3.75 & 0.71 & $<-0.09$ \\
\hline HD 16280 & 4754 & 4.39 & 0.56 & -0.19 & 4.24 & 0.71 & $<-0.39$ \\
\hline HD 168863 & 4905 & 4.40 & 0.56 & 0.16 & 4.50 & 0.82 & $<0.11$ \\
\hline HD 16905 & 4867 & 4.35 & 0.51 & 0.15 & 4.32 & 0.80 & $<0.04$ \\
\hline HD 176535 & 4727 & 4.36 & 0.54 & -0.15 & 4.02 & 0.71 & $<-0.13$ \\
\hline HD 185283 & 4848 & 4.37 & 0.27 & -0.06 & 4.71 & 0.77 & $<-0.09$ \\
\hline HD 187760 & 4618 & 4.45 & 0.23 & -0.32 & 4.36 & 0.70 & $<0.00$ \\
\hline HD 191285 & 4634 & 4.41 & 0.38 & -0.28 & 4.32 & 0.70 & $<0.09$ \\
\hline HD 191902 & 4691 & 4.25 & 0.31 & -0.18 & 4.95 & 0.72 & $<-0.09$ \\
\hline HD 193406 & 4728 & 4.50 & 0.54 & -0.34 & 4.17 & 0.70 & $<-0.02$ \\
\hline HD 200083 & 4828 & 4.42 & 0.42 & -0.09 & 4.35 & 0.75 & $<-0.09$ \\
\hline HD 200349 & 4844 & 4.50 & 0.59 & -0.26 & 4.20 & 0.71 & $<-0.22$ \\
\hline HD 202389 & 4732 & 4.43 & 0.49 & -0.25 & 4.43 & 0.71 & $<-0.30$ \\
\hline HD 202819 & 4737 & 4.40 & 0.59 & -0.26 & 4.41 & 0.70 & $<-0.08$ \\
\hline HD 207699 & 4874 & 4.36 & 0.63 & -0.12 & 4.48 & 0.76 & $<0.01$ \\
\hline HD 210573 & 4918 & 4.48 & 0.68 & -0.07 & 4.31 & 0.77 & $<-0.15$ \\
\hline HD 211583 & 4761 & 4.39 & 0.26 & 0.05 & 4.02 & 0.75 & $<0.14$ \\
\hline HD 214383 & 4876 & 4.47 & 0.59 & -0.16 & 4.08 & 0.74 & $<0.28$ \\
\hline HD 215722 & 4728 & 4.40 & 0.71 & -0.10 & 3.95 & 0.71 & $<-0.13$ \\
\hline HD 219495 & 4787 & 4.40 & 0.93 & 0.13 & 4.22 & 0.77 & $<0.16$ \\
\hline HD 224432 & 4828 & 4.38 & 0.58 & -0.06 & 4.39 & 0.76 & $<-0.20$ \\
\hline HD 22897 & 4837 & 4.44 & 0.69 & -0.25 & 4.22 & 0.71 & $<0.19$ \\
\hline HD 23472 & 4813 & 4.38 & 0.43 & -0.19 & 4.60 & 0.73 & $<-0.02$ \\
\hline HD 297396 & 4717 & 4.30 & 0.46 & 0.06 & 4.26 & 0.75 & $<-0.12$ \\
\hline HD 29985 & 4678 & 4.39 & 0.65 & -0.22 & 4.01 & 0.70 & $<0.03$ \\
\hline HD 30523 & 4662 & 4.57 & 0.79 & -0.16 & 3.96 & 0.70 & $<0.09$ \\
\hline HD 42505 & 4738 & 4.40 & 0.61 & -0.22 & 4.09 & 0.70 & $<0.17$ \\
\hline HD 45977 & 4689 & 4.30 & 0.52 & 0.03 & 4.47 & 0.75 & $<-0.14$ \\
\hline HD 4838 & 4704 & 4.63 & 0.76 & -0.21 & 4.22 & 0.70 & $<-0.04$ \\
\hline HD 73583 & 4695 & 4.50 & 0.71 & -0.21 & 4.53 & 0.71 & $<-0.08$ \\
\hline BD -002387 & 4833 & 4.47 & 0.85 & 0.03 & 4.06 & 0.76 & $<0.02$ \\
\hline BD -010184 & 4728 & 4.34 & 0.40 & -0.34 & 4.55 & 0.70 & $<-0.14$ \\
\hline BD -044138 & 4604 & 4.39 & 0.38 & -0.11 & 3.92 & 0.70 & $<0.06$ \\
\hline BD -053176 & 4758 & 4.39 & 0.82 & -0.09 & 4.24 & 0.73 & $<0.04$ \\
\hline
\end{tabular}


A\&A 576, A69 (2015)

Table 6. continued.

\begin{tabular}{|c|c|c|c|c|c|c|c|}
\hline Star & $\begin{array}{l}T_{\text {eff }} \\
(\mathrm{K})\end{array}$ & $\begin{array}{c}\log g \\
\left(\mathrm{~cm} \mathrm{~s}^{-2}\right)\end{array}$ & $\begin{array}{c}\xi_{t} \\
\left(\mathrm{~km} \mathrm{~s}^{-1}\right)\end{array}$ & {$[\mathrm{Fe} / \mathrm{H}]$} & $\begin{array}{c}\text { Age } \\
(\mathrm{Gyr})\end{array}$ & $\begin{array}{l}\text { Mass } \\
\left(M_{\odot}\right) \\
\end{array}$ & $A(\mathrm{Li})$ \\
\hline BD -053596 & 4594 & 4.32 & 0.27 & -0.34 & 4.36 & 0.70 & $<-0.19$ \\
\hline BD -063481 & 4815 & 4.36 & 0.41 & -0.14 & 4.62 & 0.74 & $<-0.50$ \\
\hline BD -064196 & 4688 & 4.30 & 0.42 & -0.03 & 4.68 & 0.75 & $<-0.18$ \\
\hline BD -064756 & 4646 & 4.54 & 0.70 & -0.23 & 4.18 & 0.70 & $<-0.25$ \\
\hline BD -090872 & 4660 & 4.35 & 0.77 & -0.19 & 4.18 & 0.70 & $<-0.23$ \\
\hline BD -092670 & 4806 & 4.46 & 0.51 & -0.08 & 4.17 & 0.74 & $<-0.12$ \\
\hline BD -094191 & 4804 & 4.44 & 0.63 & -0.32 & 4.52 & 0.71 & $<-0.08$ \\
\hline BD -130116 & 4615 & 4.33 & 0.61 & -0.49 & 4.20 & 0.70 & $<0.29$ \\
\hline BD -140184 & 4714 & 4.32 & 0.76 & -0.38 & 4.52 & 0.70 & $<0.04$ \\
\hline BD -145003 & 4640 & 4.41 & 0.35 & -0.51 & 4.32 & 0.70 & $<-0.14$ \\
\hline BD -173242 & 4627 & 4.48 & 0.31 & -0.29 & 4.32 & 0.70 & $<-0.09$ \\
\hline BD -195953 & 4775 & 4.51 & 0.71 & -0.14 & 4.44 & 0.73 & $<0.06$ \\
\hline BD -213153 & 4622 & 4.38 & 0.76 & -0.17 & 4.20 & 0.70 & $<0.31$ \\
\hline BD -223528 & 4747 & 4.28 & 0.63 & -0.17 & 4.26 & 0.71 & $<-0.19$ \\
\hline HIP 102025 & 4684 & 4.32 & 0.53 & -0.28 & 4.70 & 0.70 & $<-0.14$ \\
\hline HIP 102964 & 4797 & 4.43 & 0.72 & -0.23 & 4.43 & 0.72 & $<-0.16$ \\
\hline HIP 103867 & 4559 & 4.14 & 0.77 & -0.45 & 4.24 & 0.70 & $<-0.25$ \\
\hline HIP 105506 & 4840 & 4.37 & 0.22 & -0.01 & 4.27 & 0.76 & $<-0.18$ \\
\hline HIP 109149 & 4960 & 4.43 & 0.32 & -0.12 & 3.75 & 0.75 & $<0.16$ \\
\hline HIP 109421 & 4576 & 4.42 & 0.32 & -0.27 & 4.31 & 0.70 & $<-0.35$ \\
\hline HIP 113596 & 4580 & 4.25 & 0.67 & -0.21 & 4.27 & 0.70 & $<-0.26$ \\
\hline HIP 116374 & 4626 & 4.31 & 0.42 & 0.01 & 3.99 & 0.70 & $<-0.12$ \\
\hline HIP 17346 & 4699 & 4.32 & 0.66 & -0.16 & 4.16 & 0.70 & $<-0.08$ \\
\hline HIP 18918 & 4572 & 4.28 & 0.45 & -0.20 & 4.40 & 0.70 & $<-0.11$ \\
\hline HIP 25612 & 4571 & 4.35 & 0.56 & -0.50 & 4.28 & 0.70 & $<-0.10$ \\
\hline HIP 26013 & 4891 & 4.41 & 0.90 & 0.02 & 4.34 & 0.79 & $<-0.14$ \\
\hline HIP 36347 & 4719 & 4.38 & 0.84 & -0.05 & 3.84 & 0.72 & $<-0.06$ \\
\hline HIP 38324 & 4615 & 4.39 & 0.48 & -0.30 & 4.30 & 0.70 & $<-0.18$ \\
\hline HIP 39470 & 4571 & 4.40 & 0.61 & -0.39 & 4.17 & 0.70 & $<-0.23$ \\
\hline HIP 45301 & 4788 & 4.46 & 0.10 & -0.12 & 4.45 & 0.74 & $<0.06$ \\
\hline HIP 54597 & 4799 & 4.43 & 0.52 & -0.22 & 4.28 & 0.71 & $<0.73$ \\
\hline HIP 57688 & 4712 & 4.32 & 0.37 & -0.14 & 4.17 & 0.71 & $<-0.72$ \\
\hline HIP 58348 & 4828 & 4.34 & 0.43 & 0.00 & 4.20 & 0.76 & $<-0.18$ \\
\hline HIP 59925 & 4590 & 4.40 & 0.43 & -0.26 & 4.30 & 0.70 & $<-0.14$ \\
\hline HIP 61406 & 4855 & 4.42 & 0.68 & 0.03 & 4.19 & 0.77 & $<0.02$ \\
\hline HIP 67126 & 4681 & 4.20 & 0.27 & -0.19 & 5.02 & 0.72 & $<-0.24$ \\
\hline HIP 7058 & 4749 & 4.55 & 0.41 & -0.10 & 3.79 & 0.71 & $<-0.07$ \\
\hline HIP 7743 & 4692 & 4.39 & 0.74 & -0.17 & 4.60 & 0.71 & $<-0.16$ \\
\hline HIP 96240 & 4849 & 4.47 & 0.70 & -0.15 & 4.32 & 0.74 & $<-0.38$ \\
\hline HD 283 & 5157 & 4.51 & 0.45 & -0.54 & 5.30 & 0.72 & $<0.07$ \\
\hline HD 750 & 5060 & 4.39 & 0.59 & -0.29 & 5.20 & 0.75 & $<0.39$ \\
\hline HD 870 & 5381 & 4.42 & 0.79 & -0.10 & 3.04 & 0.87 & $<0.13$ \\
\hline HD 2025 & 4939 & 4.58 & 0.61 & -0.35 & 4.29 & 0.72 & $<-0.23$ \\
\hline HD 3569 & 5155 & 4.54 & 0.60 & -0.32 & 4.91 & 0.76 & $<0.03$ \\
\hline HD 6348 & 5107 & 4.51 & 0.07 & -0.56 & 5.16 & 0.71 & $<-0.69$ \\
\hline HD 6673 & 4960 & 4.49 & 0.58 & -0.26 & 4.48 & 0.74 & $<-0.21$ \\
\hline HD 8326 & 4971 & 4.48 & 0.81 & 0.02 & 4.41 & 0.80 & $<0.09$ \\
\hline HD 8389A & 5283 & 4.37 & 1.06 & 0.34 & 5.37 & 0.91 & $<0.73$ \\
\hline HD 8828 & 5403 & 4.46 & 0.72 & -0.16 & 4.95 & 0.85 & $<0.20$ \\
\hline HD 8859 & 5502 & 4.41 & 0.77 & -0.09 & 4.89 & 0.89 & $<0.66$ \\
\hline HD 8912 & 5211 & 4.43 & 0.70 & -0.07 & 3.64 & 0.83 & $<0.18$ \\
\hline HD 9246 & 4999 & 4.49 & 0.13 & -0.53 & 4.27 & 0.70 & $<0.30$ \\
\hline HD 9796 & 5179 & 4.38 & 0.66 & -0.25 & 4.91 & 0.78 & $<0.17$ \\
\hline HD 10002 & 5313 & 4.40 & 0.82 & 0.17 & 4.69 & 0.91 & $<0.49$ \\
\hline HD 10166 & 5221 & 4.48 & 0.74 & -0.39 & 3.37 & 0.76 & $<0.29$ \\
\hline HD 11683 & 5007 & 4.42 & 0.60 & -0.21 & 4.75 & 0.76 & 1.37 \\
\hline HD 12345 & 5395 & 4.44 & 0.69 & -0.21 & 4.46 & 0.84 & $<0.05$ \\
\hline HD 12617 & 4890 & 4.46 & 0.75 & 0.10 & 4.29 & 0.79 & $<0.02$ \\
\hline HD 13060 & 5255 & 4.34 & 0.82 & 0.02 & 3.75 & 0.86 & $<-0.31$ \\
\hline HD 13789 & 4740 & 4.33 & 0.79 & -0.06 & 4.60 & 0.75 & $<-0.30$ \\
\hline HD 14374 & 5425 & 4.48 & 0.81 & -0.04 & 4.00 & 0.89 & $<0.75$ \\
\hline HD 14635 & 4806 & 4.45 & 0.78 & -0.03 & 3.95 & 0.74 & $<0.10$ \\
\hline HD 14680 & 5011 & 4.46 & 0.69 & -0.17 & 4.32 & 0.77 & $<0.35$ \\
\hline HD 14744 & 4923 & 4.45 & 0.44 & -0.13 & 5.01 & 0.77 & $<-0.06$ \\
\hline HD 15337 & 5179 & 4.39 & 0.70 & 0.06 & 3.89 & 0.85 & $<0.42$ \\
\hline
\end{tabular}


Table 6. continued.

\begin{tabular}{|c|c|c|c|c|c|c|c|}
\hline Star & $\begin{array}{l}T_{\text {eff }} \\
(\mathrm{K})\end{array}$ & $\begin{array}{c}\log g \\
\left(\mathrm{~cm} \mathrm{~s}^{-2}\right)\end{array}$ & $\begin{array}{c}\xi_{t} \\
\left(\mathrm{~km} \mathrm{~s}^{-1}\right)\end{array}$ & {$[\mathrm{Fe} / \mathrm{H}]$} & $\begin{array}{l}\text { Age } \\
(\mathrm{Gyr})\end{array}$ & $\begin{array}{l}\text { Mass } \\
\left(M_{\odot}\right)\end{array}$ & $A(\mathrm{Li})$ \\
\hline HD 16270 & 4786 & 4.39 & 0.84 & 0.06 & 4.38 & 0.77 & $<0.03$ \\
\hline HD 16297 & 5422 & 4.47 & 0.80 & -0.01 & 5.33 & 0.89 & $<0.21$ \\
\hline HD 16714 & 5518 & 4.42 & 0.76 & -0.20 & 7.52 & 0.85 & $<0.23$ \\
\hline HD 18386 & 5457 & 4.39 & 0.92 & 0.14 & 2.13 & 0.95 & $<0.42$ \\
\hline HD 18719 & 5241 & 4.41 & 0.92 & -0.08 & 5.07 & 0.83 & $<0.21$ \\
\hline HD 21019 & 5468 & 3.93 & 1.05 & -0.45 & 6.58 & 1.07 & 1.39 \\
\hline HD 21209A & 4671 & 4.31 & 0.54 & -0.41 & 4.46 & 0.70 & $<-0.56$ \\
\hline HD 21411 & 5473 & 4.51 & 0.81 & -0.26 & 8.36 & 0.82 & $<0.78$ \\
\hline HD 21749 & 4723 & 4.40 & 0.53 & -0.02 & 4.32 & 0.73 & $<-0.09$ \\
\hline HD 22610 & 5043 & 4.44 & 0.88 & -0.22 & 4.14 & 0.76 & $<-0.21$ \\
\hline HD 23356 & 5004 & 4.50 & 0.87 & -0.17 & 4.12 & 0.77 & $<-0.09$ \\
\hline HD 24331 & 4965 & 4.51 & 0.53 & -0.31 & 4.78 & 0.73 & $<-0.04$ \\
\hline HD 25105 & 5316 & 4.47 & 0.77 & -0.15 & 4.57 & 0.83 & $<-0.04$ \\
\hline HD 25120 & 5134 & 4.47 & 0.87 & -0.18 & 4.84 & 0.79 & $<0.61$ \\
\hline HD 25565 & 5212 & 4.47 & 0.80 & 0.03 & 3.63 & 0.85 & $<0.38$ \\
\hline HD 25673 & 5136 & 4.47 & 0.56 & -0.50 & 4.20 & 0.73 & $<0.04$ \\
\hline HD 30278 & 5394 & 4.39 & 0.72 & -0.17 & 10.37 & 0.83 & $<0.23$ \\
\hline HD 30306 & 5529 & 4.32 & 0.89 & 0.17 & 9.78 & 0.94 & $<0.52$ \\
\hline HD 31560 & 4751 & 4.33 & 0.64 & -0.07 & 4.71 & 0.73 & $<-0.13$ \\
\hline HD 33725 & 5274 & 4.41 & 0.71 & -0.17 & 6.91 & 0.82 & $<0.16$ \\
\hline HD 34688 & 5169 & 4.44 & 0.70 & -0.20 & 4.35 & 0.79 & $<0.22$ \\
\hline HD 35854 & 4928 & 4.46 & 0.54 & -0.13 & 4.95 & 0.77 & $<-0.22$ \\
\hline HD 37986 & 5507 & 4.29 & 0.92 & 0.26 & 3.61 & 0.97 & $<0.65$ \\
\hline HD 44573 & 5071 & 4.48 & 0.80 & -0.07 & 4.06 & 0.80 & $<-0.01$ \\
\hline HD 48611 & 5337 & 4.51 & 0.69 & -0.36 & 4.47 & 0.79 & $<0.24$ \\
\hline HD 52919 & 4698 & 4.37 & 0.67 & -0.17 & 4.33 & 0.71 & $<-0.28$ \\
\hline HD 65277 & 4802 & 4.43 & 0.55 & -0.31 & 4.48 & 0.71 & $<-0.14$ \\
\hline HD 68607 & 5215 & 4.41 & 0.82 & 0.07 & 4.31 & 0.86 & $<0.03$ \\
\hline HD 71835 & 5438 & 4.39 & 0.79 & -0.04 & 5.91 & 0.88 & $<0.63$ \\
\hline HD 72579 & 5449 & 4.27 & 0.84 & 0.20 & 9.46 & 0.92 & $<0.61$ \\
\hline HD 72673 & 5243 & 4.46 & 0.60 & -0.41 & 10.26 & 0.75 & $<-0.17$ \\
\hline HD 74014 & 5561 & 4.33 & 0.90 & 0.22 & 5.23 & 0.98 & $<0.32$ \\
\hline HD 80883 & 5233 & 4.44 & 0.80 & -0.25 & 6.31 & 0.80 & $<0.28$ \\
\hline HD 81639 & 5522 & 4.40 & 0.79 & -0.17 & 7.26 & 0.86 & $<0.60$ \\
\hline HD 82516 & 5104 & 4.46 & 0.71 & 0.01 & 5.05 & 0.83 & $<0.02$ \\
\hline HD 85119 & 5425 & 4.52 & 0.93 & -0.20 & 3.12 & 0.85 & 1.38 \\
\hline HD 86065 & 5026 & 4.50 & 0.91 & -0.06 & 3.72 & 0.79 & $<0.07$ \\
\hline HD 86140 & 4903 & 4.55 & 0.31 & -0.25 & 4.19 & 0.73 & $<-0.22$ \\
\hline HD 86171 & 5400 & 4.47 & 0.81 & -0.25 & 4.28 & 0.83 & $<0.98$ \\
\hline HD 87521 & 4854 & 4.37 & 0.76 & -0.04 & 4.28 & 0.76 & $<0.07$ \\
\hline HD 88656 & 5150 & 4.44 & 0.81 & -0.11 & 5.53 & 0.81 & $<0.24$ \\
\hline HD 90711 & 5444 & 4.40 & 0.92 & 0.24 & 2.74 & 0.95 & $<0.58$ \\
\hline HD 90812 & 5164 & 4.48 & 0.64 & -0.36 & 4.28 & 0.75 & $<-0.06$ \\
\hline HD 94151 & 5583 & 4.38 & 0.83 & 0.04 & 8.38 & 0.92 & $<0.42$ \\
\hline HD 97343 & 5410 & 4.39 & 0.82 & -0.06 & 10.39 & 0.85 & $<0.12$ \\
\hline HD 98281 & 5381 & 4.42 & 0.64 & -0.26 & 8.85 & 0.81 & $<0.04$ \\
\hline HD 98356 & 5322 & 4.41 & 0.84 & 0.10 & 4.19 & 0.89 & $<0.53$ \\
\hline HD 100508 & 5449 & 4.42 & 0.86 & 0.39 & 5.36 & 0.95 & $<0.59$ \\
\hline HD 101581 & 4738 & 4.46 & 0.66 & -0.52 & 4.13 & 0.70 & $<-0.29$ \\
\hline HD 102438 & 5560 & 4.41 & 0.84 & -0.29 & 11.40 & 0.82 & $<-0.08$ \\
\hline HD 103949 & 4881 & 4.48 & 0.49 & -0.07 & 4.20 & 0.76 & $<-0.17$ \\
\hline HD 105671 & 4748 & 4.42 & 0.90 & -0.02 & 4.13 & 0.73 & $<-0.08$ \\
\hline HD 106275 & 5059 & 4.47 & 0.67 & -0.09 & 5.18 & 0.80 & $<-0.03$ \\
\hline HD 109200 & 5134 & 4.51 & 0.68 & -0.31 & 6.92 & 0.76 & $<-0.14$ \\
\hline HD 109423 & 5074 & 4.44 & 0.87 & -0.07 & 3.53 & 0.80 & $<-0.31$ \\
\hline HD 112540 & 5523 & 4.52 & 0.74 & -0.17 & 5.75 & 0.87 & $<0.28$ \\
\hline HD 116858 & 4990 & 4.52 & 0.77 & -0.21 & 4.63 & 0.76 & $<-0.24$ \\
\hline HD 116920 & 5015 & 4.46 & 0.68 & -0.23 & 4.36 & 0.76 & $<-0.17$ \\
\hline HD 119782 & 5160 & 4.44 & 0.79 & -0.07 & 6.01 & 0.82 & $<-0.42$ \\
\hline HD 124106 & 5106 & 4.49 & 0.80 & -0.17 & 5.06 & 0.79 & $<-0.88$ \\
\hline HD 124292 & 5443 & 4.37 & 0.77 & -0.13 & 10.39 & 0.85 & $<-0.50$ \\
\hline HD 124364 & 5584 & 4.48 & 0.83 & -0.27 & 4.05 & 0.86 & 1.23 \\
\hline HD 125072 & 5007 & 4.56 & 1.04 & 0.18 & 4.25 & 0.82 & $<0.36$ \\
\hline HD 125455 & 5162 & 4.52 & 0.70 & -0.18 & 5.62 & 0.80 & $<-0.64$ \\
\hline HD 128674 & 5551 & 4.50 & 0.71 & -0.38 & 10.90 & 0.80 & $<0.86$ \\
\hline
\end{tabular}


Table 6. continued.

\begin{tabular}{|c|c|c|c|c|c|c|c|}
\hline Star & $\begin{array}{l}T_{\text {eff }} \\
(\mathrm{K}) \\
\end{array}$ & $\begin{array}{c}\log g \\
\left(\mathrm{~cm} \mathrm{~s}^{-2}\right)\end{array}$ & $\begin{array}{c}\xi_{t} \\
\left(\mathrm{~km} \mathrm{~s}^{-1}\right)\end{array}$ & {$[\mathrm{Fe} / \mathrm{H}]$} & $\begin{array}{r}\text { Age } \\
(\mathrm{Gyr}) \\
\end{array}$ & $\begin{array}{l}\text { Mass } \\
\left(M_{\odot}\right) \\
\end{array}$ & $A(\mathrm{Li})$ \\
\hline HD 130992 & 4898 & 4.54 & 0.71 & -0.13 & 4.04 & 0.75 & $<-0.20$ \\
\hline HD 132648 & 5418 & 4.49 & 0.69 & -0.37 & 9.55 & 0.78 & $<0.52$ \\
\hline HD 136713 & 4994 & 4.45 & 0.94 & 0.07 & 4.33 & 0.81 & $<0.10$ \\
\hline HD 136894 & 5412 & 4.36 & 0.75 & -0.10 & 9.74 & 0.85 & $<-0.36$ \\
\hline HD 137303 & 4756 & 4.51 & 0.40 & -0.35 & 4.62 & 0.70 & $<-0.41$ \\
\hline HD 142709 & 4728 & 4.44 & 0.84 & -0.35 & 4.35 & 0.70 & $<-0.22$ \\
\hline HD 143295 & 4987 & 4.43 & 0.89 & -0.03 & 3.99 & 0.79 & 1.24 \\
\hline HD 144411 & 4852 & 4.39 & 0.05 & -0.32 & 4.28 & 0.71 & $<-0.28$ \\
\hline HD 144497 & 5022 & 4.50 & 0.82 & -0.12 & 4.48 & 0.78 & -0.28 \\
\hline HD 144628 & 5085 & 4.51 & 0.55 & -0.41 & 5.16 & 0.74 & $<-0.29$ \\
\hline HD 147512 & 5530 & 4.40 & 0.81 & -0.08 & 9.93 & 0.87 & $<0.40$ \\
\hline HD 148303 & 4958 & 4.55 & 0.84 & -0.03 & 4.41 & 0.79 & $<0.86$ \\
\hline HD 151504 & 5457 & 4.36 & 0.87 & 0.06 & 8.73 & 0.90 & $<0.47$ \\
\hline HD 153851 & 5052 & 4.50 & 0.91 & -0.25 & 4.80 & 0.76 & $<0.06$ \\
\hline HD 154577 & 4900 & 4.52 & 0.42 & -0.70 & 6.02 & 0.70 & $<-0.51$ \\
\hline HD 157830 & 5540 & 4.49 & 0.76 & -0.25 & 5.12 & 0.85 & $<0.08$ \\
\hline HD 161098 & 5560 & 4.46 & 0.79 & -0.27 & 8.16 & 0.84 & $<0.47$ \\
\hline HD 162236 & 5343 & 4.43 & 0.82 & -0.12 & 4.20 & 0.85 & $<0.09$ \\
\hline HD 165920 & 5339 & 4.39 & 0.79 & 0.29 & 5.14 & 0.92 & $<0.43$ \\
\hline HD 167359 & 5348 & 4.46 & 0.67 & -0.19 & 4.51 & 0.83 & $<0.05$ \\
\hline HD 168159 & 4783 & 4.42 & 0.99 & -0.15 & 4.05 & 0.72 & 0.91 \\
\hline HD 170493 & 4751 & 4.24 & 0.59 & 0.14 & 4.40 & 0.78 & $<-0.04$ \\
\hline HD 172513 & 5500 & 4.41 & 0.79 & -0.05 & 4.36 & 0.90 & $<0.46$ \\
\hline HD 176157 & 5181 & 4.41 & 0.92 & -0.16 & 5.00 & 0.81 & $<0.19$ \\
\hline HD 176986 & 5018 & 4.45 & 0.82 & 0.00 & 4.47 & 0.81 & $<0.13$ \\
\hline HD 183783 & 4595 & 4.29 & 0.05 & -0.20 & 4.93 & 0.71 & $<-0.39$ \\
\hline HD 183870 & 5029 & 4.49 & 0.78 & -0.07 & 4.92 & 0.79 & $<-0.23$ \\
\hline HD 186061 & 5016 & 4.51 & 0.62 & -0.02 & 4.07 & 0.80 & $<0.19$ \\
\hline HD 188559 & 4786 & 4.33 & 0.65 & -0.11 & 4.39 & 0.74 & $<-0.27$ \\
\hline HD 189242 & 4913 & 4.46 & 0.56 & -0.38 & 5.14 & 0.72 & $<-0.26$ \\
\hline HD 191847 & 5066 & 4.45 & 0.48 & -0.12 & 4.17 & 0.79 & $<-0.14$ \\
\hline HD 192117 & 5479 & 4.48 & 0.75 & -0.04 & 3.54 & 0.90 & $<0.78$ \\
\hline HD 192961 & 4624 & 4.31 & 0.58 & -0.35 & 4.32 & 0.70 & $<-0.11$ \\
\hline HD 193844 & 5007 & 4.44 & 0.48 & -0.30 & 4.47 & 0.74 & $<-0.18$ \\
\hline HD 195302 & 5063 & 4.44 & 0.64 & 0.02 & 4.32 & 0.82 & $<-0.12$ \\
\hline HD 196761 & 5415 & 4.43 & 0.76 & -0.31 & 11.29 & 0.79 & $<0.13$ \\
\hline HD 197210 & 5577 & 4.42 & 0.86 & -0.03 & 3.70 & 0.93 & $<0.51$ \\
\hline HD 197823 & 5396 & 4.41 & 0.82 & 0.12 & 6.64 & 0.91 & $<0.29$ \\
\hline HD 199933 & 4730 & 4.32 & 0.64 & -0.15 & 4.37 & 0.72 & $<-0.09$ \\
\hline HD 200505 & 5052 & 4.47 & 0.73 & -0.45 & 4.62 & 0.72 & $<0.18$ \\
\hline HD 203384 & 5586 & 4.40 & 0.90 & 0.26 & 2.77 & 1.00 & $<0.57$ \\
\hline HD 203413 & 4812 & 4.39 & 0.74 & 0.01 & 4.38 & 0.76 & $<-0.13$ \\
\hline HD 203850 & 4879 & 4.51 & 0.36 & -0.68 & 4.93 & 0.70 & $<-0.60$ \\
\hline HD 205536 & 5442 & 4.38 & 0.77 & -0.05 & 9.37 & 0.87 & $<0.10$ \\
\hline HD 206163 & 5519 & 4.43 & 0.94 & 0.01 & 2.20 & 0.94 & $<0.58$ \\
\hline HD 207583 & 5534 & 4.46 & 0.99 & 0.01 & 2.99 & 0.93 & $<1.35$ \\
\hline HD 208272 & 5199 & 4.42 & 0.99 & -0.08 & 5.31 & 0.83 & $<-0.23$ \\
\hline HD 208573 & 4910 & 4.41 & 0.86 & 0.00 & 4.31 & 0.78 & $<-0.16$ \\
\hline HD 209100 & 4754 & 4.45 & 0.68 & -0.20 & 4.18 & 0.71 & $<-0.39$ \\
\hline HD 209742 & 5137 & 4.49 & 0.79 & -0.16 & 4.93 & 0.80 & $<-0.14$ \\
\hline HD 211369 & 4984 & 4.44 & 0.67 & 0.04 & 4.15 & 0.80 & $<0.12$ \\
\hline HD 212563 & 5018 & 4.52 & 0.89 & -0.02 & 4.07 & 0.80 & 1.56 \\
\hline HD 212580 & 5155 & 4.44 & 0.85 & -0.11 & 4.05 & 0.81 & $<-0.01$ \\
\hline HD 213628 & 5555 & 4.44 & 0.82 & 0.01 & 3.07 & 0.94 & $<0.73$ \\
\hline HD 214759 & 5461 & 4.37 & 0.85 & 0.18 & 3.51 & 0.95 & $<0.54$ \\
\hline HD 218249 & 5009 & 4.52 & 0.46 & -0.40 & 4.31 & 0.72 & $<0.09$ \\
\hline HD 218511 & 4556 & 4.31 & 0.41 & -0.10 & 4.40 & 0.70 & $<-0.14$ \\
\hline HD 218572 & 4785 & 4.54 & 0.50 & -0.56 & 4.32 & 0.70 & $<0.16$ \\
\hline HD 219249 & 5482 & 4.50 & 0.74 & -0.40 & 8.16 & 0.79 & $<0.55$ \\
\hline HD 220339 & 5029 & 4.55 & 0.76 & -0.35 & 4.93 & 0.74 & $<-0.31$ \\
\hline HD 222335 & 5271 & 4.49 & 0.83 & -0.20 & 5.86 & 0.81 & $<0.16$ \\
\hline HD 222422 & 5475 & 4.46 & 0.73 & -0.12 & 3.58 & 0.88 & $<0.91$ \\
\hline HD 223121 & 5077 & 4.34 & 0.74 & 0.05 & 4.15 & 0.82 & $<0.49$ \\
\hline HD 223282 & 5328 & 4.49 & 0.60 & -0.41 & 5.93 & 0.77 & $<0.48$ \\
\hline HD 224619 & 5436 & 4.39 & 0.79 & -0.20 & 9.49 & 0.83 & $<0.19$ \\
\hline
\end{tabular}


Table 6. continued.

\begin{tabular}{|c|c|c|c|c|c|c|c|}
\hline Star & $\begin{array}{l}T_{\text {eff }} \\
(\mathrm{K})\end{array}$ & $\begin{array}{c}\log g \\
\left(\mathrm{~cm} \mathrm{~s}^{-2}\right)\end{array}$ & $\begin{array}{c}\xi_{t} \\
\left(\mathrm{~km} \mathrm{~s}^{-1}\right)\end{array}$ & {$[\mathrm{Fe} / \mathrm{H}]$} & $\begin{array}{r}\text { Age } \\
(\mathrm{Gyr}) \\
\end{array}$ & $\begin{array}{l}\text { Mass } \\
\left(M_{\odot}\right)\end{array}$ & $A(\mathrm{Li})$ \\
\hline HD 224789 & 5185 & 4.44 & 1.05 & -0.03 & 4.86 & 0.83 & $<0.06$ \\
\hline HD 171587 & 5412 & 4.59 & 0.76 & -0.64 & 7.40 & 0.77 & 1.26 \\
\hline HD 207869 & 5527 & 4.50 & 0.73 & -0.45 & 4.28 & 0.81 & $<0.44$ \\
\hline HD 113101 & 5456 & 4.37 & 0.66 & -0.07 & 4.92 & 0.89 & $<0.54$ \\
\hline HD 11397 & 5565 & 4.50 & 0.77 & -0.55 & 5.56 & 0.80 & $<-0.07$ \\
\hline HD 126681 & 5561 & 4.71 & 0.71 & -1.14 & 4.68 & 0.71 & 1.52 \\
\hline HD 126803 & 5477 & 4.50 & 0.50 & -0.61 & 6.31 & 0.78 & $<-0.28$ \\
\hline HD 137676 & 5253 & 3.93 & 0.74 & -0.53 & 11.19 & 0.93 & $<1.16$ \\
\hline HD 139189 & 5075 & 4.41 & 0.78 & 0.02 & 4.60 & 0.83 & $<-0.92$ \\
\hline HD 139332 & 4899 & 4.30 & 0.04 & 0.00 & 4.34 & 0.79 & $<-0.53$ \\
\hline HD 139536 & 5209 & 4.71 & 1.13 & -0.04 & 4.27 & 0.84 & $<-0.46$ \\
\hline HD 14452 & 5313 & 4.50 & 0.85 & -0.16 & 4.53 & 0.84 & $<0.34$ \\
\hline HD 163436 & 5030 & 4.43 & 0.45 & -0.07 & 4.42 & 0.80 & $<-0.42$ \\
\hline HD 197921 & 4913 & 4.36 & 0.34 & 0.13 & 4.57 & 0.82 & $<-0.24$ \\
\hline HD 210320 & 5597 & 4.31 & 0.87 & 0.11 & 6.59 & 0.96 & $<0.56$ \\
\hline HD 218750 & 5166 & 4.39 & 0.57 & 0.08 & 4.80 & 0.86 & $<-0.26$ \\
\hline HD 223272 & 5118 & 4.41 & 0.58 & 0.13 & 4.35 & 0.85 & $<-0.08$ \\
\hline HD 224063 & 5591 & 4.27 & 0.84 & 0.14 & 7.51 & 0.97 & $<0.19$ \\
\hline HD 23901 & 5264 & 3.93 & 0.84 & -0.40 & 11.17 & 0.93 & $<1.10$ \\
\hline HD 24633 & 5276 & 4.36 & 0.59 & -0.04 & 5.90 & 0.87 & $<-0.20$ \\
\hline HD 291763 & 4987 & 4.50 & 0.12 & -0.61 & 4.44 & 0.70 & $<-0.35$ \\
\hline HD 324492 & 4962 & 4.15 & 0.52 & -0.27 & 4.19 & 0.73 & $<-0.87$ \\
\hline HD 329788 & 5151 & 4.35 & 0.19 & -0.08 & 4.00 & 0.81 & $<0.11$ \\
\hline HD 56380 & 5317 & 4.35 & 0.53 & -0.42 & 6.28 & 0.79 & $<0.03$ \\
\hline HD 61051 & 5363 & 4.37 & 0.70 & -0.10 & 4.57 & 0.86 & $<-0.11$ \\
\hline HD 63685 & 5497 & 4.05 & 0.94 & 0.00 & 9.78 & 1.01 & $<0.66$ \\
\hline HD 75530 & 5311 & 4.48 & 0.53 & -0.54 & 6.34 & 0.77 & $<-0.33$ \\
\hline HD 82783 & 5318 & 4.41 & 0.91 & 0.21 & 3.92 & 0.91 & $<0.43$ \\
\hline $\mathrm{BD}-082534$ & 5405 & 4.43 & 0.41 & -0.78 & 5.16 & 0.73 & $<0.69$ \\
\hline HIP 104856 & 5023 & 4.43 & 0.22 & -0.24 & 4.64 & 0.76 & $<0.18$ \\
\hline HIP 31639 & 5400 & 4.53 & 0.31 & -0.54 & 4.56 & 0.77 & $<0.61$ \\
\hline HIP 32127 & 5302 & 4.44 & 0.58 & -0.64 & 5.65 & 0.75 & $<0.11$ \\
\hline HIP 41659 & 5197 & 4.38 & 0.42 & -0.53 & 5.31 & 0.75 & $<0.15$ \\
\hline HIP 88316 & 5159 & 4.38 & 0.35 & -0.01 & 4.82 & 0.84 & $<0.35$ \\
\hline HD 101650 & 4626 & 4.29 & 0.11 & -0.45 & 4.09 & 0.70 & $<-0.26$ \\
\hline HD 108935 & 4724 & 4.30 & 0.55 & 0.02 & 4.21 & 0.74 & $<-0.14$ \\
\hline HD 147147 & 4856 & 4.51 & 0.71 & -0.17 & 4.25 & 0.74 & $<0.71$ \\
\hline HD 189987 & 4746 & 4.25 & 0.09 & -0.06 & 4.92 & 0.75 & $<-0.04$ \\
\hline HD 20492 & 4770 & 4.30 & 0.59 & 0.02 & 4.38 & 0.76 & $<-0.10$ \\
\hline HD 214998 & 4847 & 4.37 & 0.77 & 0.06 & 4.24 & 0.78 & $<0.09$ \\
\hline HD 326267 & 4719 & 4.31 & 0.45 & -0.26 & 4.26 & 0.70 & $<-0.05$ \\
\hline HD 57568 & 4821 & 4.51 & 0.38 & -0.47 & 4.14 & 0.70 & $<-0.12$ \\
\hline HD 58489 & 4800 & 4.33 & 0.59 & 0.10 & 4.32 & 0.77 & $<-0.01$ \\
\hline HD 89668 & 4811 & 4.45 & 0.63 & -0.11 & 4.02 & 0.73 & $<-0.08$ \\
\hline HD 96673 & 4788 & 4.38 & 0.55 & -0.13 & 4.40 & 0.73 & $<0.03$ \\
\hline $\mathrm{BD}-012505$ & 4741 & 4.51 & 0.44 & -0.11 & 3.59 & 0.70 & $<0.21$ \\
\hline BD -034797 & 4622 & 4.21 & 0.30 & 0.06 & 4.42 & 0.73 & $<-0.02$ \\
\hline BD -050484 & 4674 & 4.42 & 0.21 & -0.39 & 4.36 & 0.70 & $<-0.09$ \\
\hline BD -120327 & 4680 & 4.35 & 0.57 & -0.34 & 4.47 & 0.70 & $<0.02$ \\
\hline BD -123458 & 4803 & 4.86 & 0.43 & -0.83 & 3.25 & 0.70 & $<-0.01$ \\
\hline BD -130321 & 4772 & 4.41 & 0.78 & 0.02 & 3.67 & 0.73 & $<0.14$ \\
\hline HIP 108216 & 4830 & 4.53 & 0.66 & -0.51 & 4.22 & 0.70 & $<-0.34$ \\
\hline HIP 21934 & 4674 & 4.19 & 0.62 & 0.03 & 3.81 & 0.71 & $<-0.04$ \\
\hline HIP 64965 & 4888 & 4.78 & 0.00 & -1.03 & 4.01 & 0.70 & $<0.07$ \\
\hline HIP 80083 & 4800 & 4.78 & 0.31 & -0.80 & 4.10 & 0.70 & $<0.14$ \\
\hline HIP 9398 & 4734 & 4.49 & 0.62 & -0.43 & 3.79 & 0.70 & $<0.04$ \\
\hline HD 967 & 5564 & 4.51 & 0.79 & -0.68 & 8.39 & 0.78 & $<0.42$ \\
\hline HD 8638 & 5507 & 4.43 & 0.74 & -0.38 & 7.91 & 0.80 & $<0.05$ \\
\hline HD 14747 & 5516 & 4.43 & 0.72 & -0.39 & 11.20 & 0.79 & $<0.32$ \\
\hline HD 17970 & 5040 & 4.39 & 0.29 & -0.45 & 9.12 & 0.74 & $<-0.29$ \\
\hline HD 19034 & 5477 & 4.40 & 0.69 & -0.48 & 9.27 & 0.77 & $<-0.06$ \\
\hline HD 24892 & 5363 & 3.99 & 0.88 & -0.32 & 11.09 & 0.95 & 1.39 \\
\hline HD 26965A & 5153 & 4.39 & 0.36 & -0.31 & 10.80 & 0.77 & $<-0.34$ \\
\hline HD 36003 & 4647 & 4.31 & 0.42 & -0.20 & 4.53 & 0.70 & $<-0.32$ \\
\hline HD 40397 & 5527 & 4.39 & 0.83 & -0.13 & 10.69 & 0.86 & $<0.52$ \\
\hline
\end{tabular}


A\&A 576, A69 (2015)

Table 6. continued.

\begin{tabular}{lcccrrrr}
\hline \hline Star & $\begin{array}{c}T_{\text {eff }} \\
(\mathrm{K})\end{array}$ & $\begin{array}{c}\log g \\
\left(\mathrm{~cm} \mathrm{~s}^{-2}\right)\end{array}$ & $\begin{array}{c}\xi_{t} \\
\left(\mathrm{~km} \mathrm{~s}^{-1}\right)\end{array}$ & $\begin{array}{r}\text { Fe/H }] \\
\text { Age } \\
(\mathrm{Gyr})\end{array}$ & $\begin{array}{r}\text { Mass } \\
\left(M_{\odot}\right)\end{array}$ & $A(\mathrm{Li})$ \\
\hline HD 50590 & 4870 & 4.39 & 0.35 & -0.22 & 4.85 & 0.74 & $<-0.21$ \\
HD 65562 & 5076 & 4.39 & 0.45 & -0.10 & 5.11 & 0.80 & $<-0.17$ \\
HD 82342 & 4820 & 4.41 & 0.30 & -0.54 & 4.41 & 0.70 & $<-0.45$ \\
HD 104006 & 5023 & 4.56 & 0.15 & -0.78 & 5.68 & 0.70 & $<-0.48$ \\
HD 104263 & 5477 & 4.34 & 0.81 & 0.02 & 9.11 & 0.89 & $<0.08$ \\
HD 114747 & 5172 & 4.44 & 0.98 & 0.21 & 3.74 & 0.87 & $<0.32$ \\
HD 123265 & 5338 & 4.29 & 0.85 & 0.19 & 4.78 & 0.91 & $<0.37$ \\
HD 129642 & 5026 & 4.49 & 0.69 & -0.06 & 4.89 & 0.81 & $<-0.13$ \\
HD 130930 & 5027 & 4.45 & 0.50 & 0.01 & 4.94 & 0.82 & $<0.01$ \\
HD 134985 & 5090 & 4.44 & 0.10 & -0.60 & 4.61 & 0.72 & $<-0.40$ \\
HD 145598 & 5417 & 4.48 & 0.59 & -0.78 & 6.19 & 0.72 & $<-0.20$ \\
HD 174545 & 5216 & 4.40 & 0.88 & 0.22 & 6.34 & 0.89 & $<0.35$ \\
HD 185615 & 5570 & 4.34 & 0.84 & 0.08 & 6.28 & 0.94 & $<0.33$ \\
HD 187456 & 4832 & 4.33 & 0.56 & 0.02 & 4.50 & 0.78 & $<-0.15$ \\
HD 190954 & 5430 & 4.46 & 0.63 & -0.41 & 7.75 & 0.78 & $<0.19$ \\
HD 192031 & 5215 & 4.39 & 0.04 & -0.84 & 6.55 & 0.70 & $<0.02$ \\
HD 207970 & 5556 & 4.38 & 0.80 & 0.07 & 7.93 & 0.93 & $<0.39$ \\
HD 210975 & 4749 & 4.37 & 0.05 & -0.43 & 4.31 & 0.70 & $<-0.54$ \\
HD 213042 & 4831 & 4.38 & 0.82 & 0.08 & 4.41 & 0.77 & $<0.06$ \\
HD 213941 & 5532 & 4.41 & 0.72 & -0.46 & 11.38 & 0.79 & $<0.38$ \\
HD 219077 & 5362 & 4.00 & 0.92 & -0.13 & 9.04 & 1.04 & 1.49 \\
HD 220256 & 5144 & 4.41 & 0.47 & -0.10 & 6.48 & 0.82 & $<-0.09$ \\
HD 222237 & 4780 & 4.37 & 0.05 & -0.38 & 4.82 & 0.70 & $<-0.40$ \\
HD 967 & 5568 & 4.53 & 0.77 & -0.68 & 8.39 & 0.78 & $<-0.18$ \\
HD 68089 & 5597 & 4.53 & 0.66 & -0.77 & 6.25 & 0.77 & $<1.01$ \\
HD 108564 & 4818 & 4.67 & 0.26 & -0.97 & 4.35 & 0.70 & $<-0.20$ \\
HD 111515 & 5398 & 4.47 & 0.71 & -0.61 & 7.59 & 0.77 & $<0.25$ \\
HD 131653 & 5324 & 4.54 & 0.35 & -0.66 & 4.48 & 0.73 & $<0.09$ \\
HD 175607 & 5392 & 4.51 & 0.60 & -0.62 & 8.14 & 0.77 & $<-0.14$ \\
CD -452997 & 5312 & 4.39 & 0.24 & -0.84 & - & - & $<0.45$ \\
\hline
\end{tabular}

